\title{
MID-IR ULTRAFAST LASER INSCRIBED WAVEGUIDES AND DEVICES
}

\author{
Dissertation \\ Submitted to \\ The School of Engineering of the \\ UNIVERSITY OF DAYTON \\ In Partial Fulfillment of the Requirements for \\ The Degree of \\ Doctor of Philosophy in Electro-Optics \\ By \\ Sean A. McDaniel \\ UNIVERSITY OF DAYTON \\ Dayton, Ohio \\ December, 2017
}




\section{MID-IR ULTRAFAST LASER INSCRIBED WAVEGUIDES AND DEVICES}

Name: McDaniel, Sean A.

APPROVED BY:

Gary Cook, Ph.D.

Advisor Committee Chairman

Adjunct Professor, Department of

Electro-Optics
Patrick Berry, Ph.D.

Committee Member

Adjunct Professor, Department of

Electro-Optics

Joseph Haus, Ph.D

Committee Member

Professor, Department of Electro-Optics

Andrew Sarangan, Ph.D

Committee Member

Professor, Department of Electro-Optics
Monish Chatterjee, Ph.D

Committee Member

Professor, Department of Electrical and Computer Engineering
Robert J. Wilkens, Ph.D., P.E.

Associate Dean for Research and

Innovation,

Professor

School of Engineering
Eddy M. Rojas, Ph.D., M.A., P. E.

Dean, School of Engineering 


\section{(c) Copyright by}

Sean A. McDaniel

All rights reserved 


\section{ABSTRACT \\ MID-IR ULTRAFAST LASER INSCRIBED WAVEGUIDES AND DEVICES}

Name: McDaniel, Sean A.

University of Dayton

Advisor: Dr. Gary Cook

Ultrafast laser inscription (ULI) is a highly versatile technique for creating index modifications in glasses and crystalline materials. The process of ULI relies on ultrashort laser pulses focused inside of a material. The high intensity of the pulsed beam induces nonlinear absorption processes, which transfers the pulse energy to the material lattice. With careful experimental control of the laser parameters, a permanent change in the refractive can be obtained in the bulk material. The permanent refractive index change obtained by ULI can be used to create waveguides in active laser materials, such as Cr:ZnSe, Fe:ZnSe and Ho:YAG.

Transition metal and rare-earth laser sources have been shown to operate over the $2-5 \mu \mathrm{m}$ range. ULI can be used in conjunction with these materials to produce high power, guided-wave structures with reduced size, weight and power (SWaP) requirements. Power levels for $\mathrm{Cr}: \mathrm{ZnSe}$ and Fe:ZnSe have been scaled to $>5 W$ and $>1 W$ respectively in ULI waveguide devices. Additionally, the first Ho:YAG ULI laser has been investigated, exhibiting output powers of $\approx 2 W$.

In addition to these advances, the theoretical limit for transition metal waveguide lasers was investigated. Transition metal lasers are highly sensitive to the operating temperature of the laser device. If the temperature increase induced in the sample is too high, phonon assisted transitions 
become dominant, thus decreasing the performance of the laser. Laser rate-equations and a thermal model for ULI waveguides were developed to establish a theoretical limit to ULI waveguide operation.

Finally, several advancements were made with respect to creating ULI waveguides. An algorithm was developed for creating arbitrary ULI structures from computer generated models. The ability to create arbitrarily generated structures provides the ability to create complex structures using ULI, such as splitters, couplers, and photonic lanterns. Furthermore, a new helical inscription technique was devised for creating smooth index profiles and for creating Bragg structuring. 


\section{ACKNOWLEDGMENTS}

I would like to express my appreciation to my advisors/mentors Dr. Gary Cook and Dr. Patrick Berry for their support and encouragement throughout my Ph.D. research. I would also like to thank the AFRL/RYDH laser sources team for their support through this effort.

Sincere thanks to Ajoy Kar, Adam Lancaster and Fiona Thorburn at Heriot-Watt University for their valuable insights into ultrafast laser inscription and for allowing us to collaborate with them on this research.

Special thanks to my parents Scott and Ann, whom I cannot thank enough for their support. I never would have made it this far without you.

I thank my beloved wife, Megan, for her unconditional support and unfailing commitment. Her support of this work has been unending, even if she understands none of what I wrote. 


\section{TABLE OF CONTENTS}

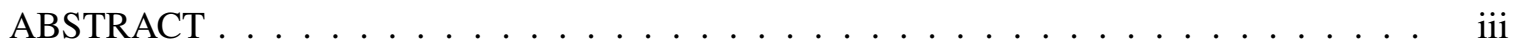

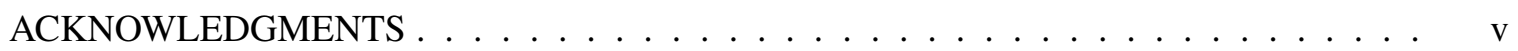

LIST OF FIGURES $\ldots \ldots \ldots \ldots \ldots \ldots \ldots \ldots \ldots$ ix

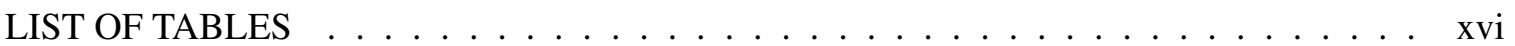

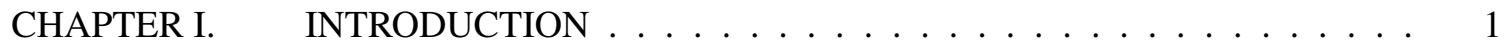

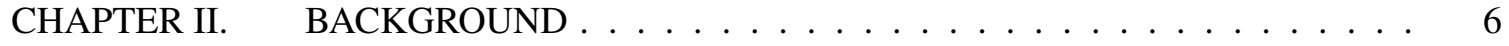

2.1 Section 1: Ultrafast Laser Inscription $\ldots \ldots \ldots \ldots$

2.2 Physical Processes . . . . . . . . . . . . . . . . . . . . . . . . 10

2.3 Transition Metal Lasers _. . . . . . . . . . . . . . . . . . . . . . . . 13

CHAPTER III. CHARACTERIZATION TECHNIQUES $\ldots \ldots \ldots \ldots \ldots \ldots$

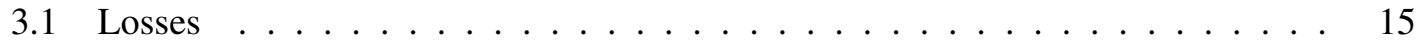

3.2 Index Difference . . . . . . . . . . . . . . . . . . . . 17

3.2.1 Direct Measurement . . . . . . . . . . . . . . . . . . 17

3.2.2 Inverse Helmholtz . . . . . . . . . . . . . . . . . . . . . . . . . 19

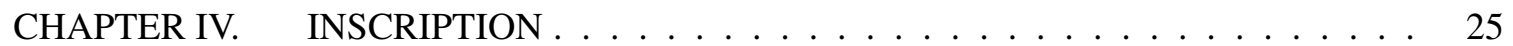

4.1 Femtosecond Laser Inscription . . . . . . . . . . . . . . . . . . . . . . . 27

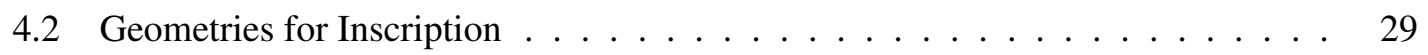

4.2.1 Longitudinal Geometry . . . . . . . . . . . . . . . . . . 29

4.2 .2 Transverse Geometry . . . . . . . . . . . . . . . . . 31

CHAPTER V. GEOMETRY GENERATION . . . . . . . . . . . . . . . . . 34

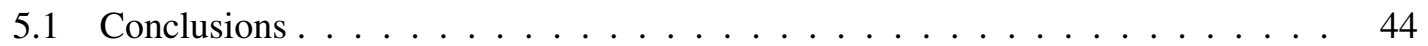


CHAPTER VI. TRANSITION METAL ULI LASERS $\ldots \ldots \ldots \ldots$

6.1 Power Scaling . . . . . . . . . . . . . . . . . . . . . . 46

6.1 .1 Fabrication . . . . . . . . . . . . . . . 48

6.1 .2 Experimental Setup . . . . . . . . . . . . . . . 51

6.1.3 Special Considerations . . . . . . . . . . . . . . . 53

$6.1 .4 \mathrm{Cr}: Z n S e$ Results . . . . . . . . . . . . . . . . . . . 55

6.1 .5 Fe:ZnSe Results . . . . . . . . . . . . . . . . . . 60

6.2 Gain Switching . . . . . . . . . . . . . . . . . . . . . . . . . . . . . . . . . . 62

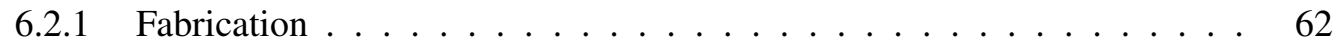

6.2 .2 Results ........................... 65

6.3 Bragg Waveguides . . . . . . . . . . . . . . . 71

CHAPTER VII. HO:YAG ULI LASERS . . . . . . . . . . . . . . . . . . 78

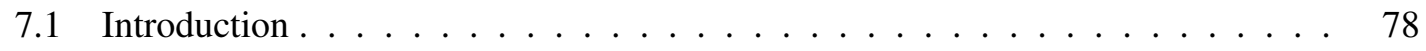

7.2 Fabrication . . . . . . . . . . . . . . . . . . 79

7.3 Experimental Setup . . . . . . . . . . . . . . . . . . . 83

7.4 Multimode Operation . . . . . . . . . . . . . . . . . . . . 84

7.5 Single Mode Operation . . . . . . . . . . . . . . . . . . . . . . . . . . . . . . . . . . 86

7.6 Mode Profiles . . . . . . . . . . . . . . . . . . . 88

7.7 Co-Doped Waveguides $\ldots \ldots \ldots \ldots$

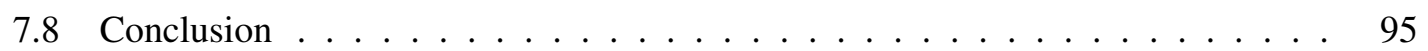

CHAPTER VIII. MODELING . . . . . . . . . . . . . . . . . . . 96

8.1 Theoretical Limit . . . . . . . . . . . . . . . . . . . . . 103

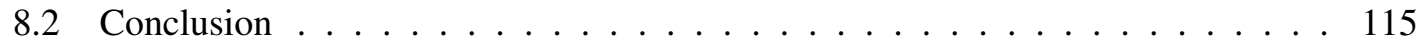

CHAPTER IX. CONCLUSIONS AND FUTURE WORK . . . . . . . . . . . . . 117

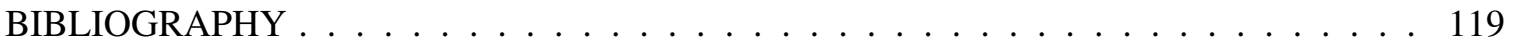
APPENDICES:

APPENDIX A. Nonlinear Devices . . . . . . . . . . . . . . . . . . . . . 129

A.1 Introduction . . . . . . . . . . . . . . . . . . . . 129

A.2 Nonlinear Processes ． . . . . . . . . . . . . . . . . . . . . . . . . . . 129

A.3 Materials of Mid-IR Stokes Generation . . . . . . . . . . . . . . . 131

A.4 Recent Progress . . . . . . . . . . . . . . . . . . . . . . 133 
APPENDIX B. Bragg Grating Theory . . . . . . . . . . . . . . 136

APPENDIX C. Arbitrary Geometry Code . . . . . . . . . . . . . . . . . . . . . . 139

APPENDIX D. Rate Equation Code . . . . . . . . . . . . . . . . . . . . . 143 


\section{LIST OF FIGURES}

2.1 Femtosecond laser modification inside of a bulk $\mathrm{ZnSe}$ crystal. The modification was created by a $700 \mathrm{fs}$ pulse focused with a 0.65 NA microscope objective. . . . . . 9

2.2 A) Type 1 modification B) Type II modification C)Type III modification $\ldots \ldots \ldots$

2.3 Energy level diagrams showing transitions from rare earth (RE) ions and transition metal (TM) ions shown in (a) and (b) respectively. This diagram has been reproduced from Petermann et al. $[41] \ldots \ldots \ldots \ldots$

3.1 Scattered intensity image from a ULI waveguide in ZnSe captured using a FLIR SC6700 thermal camera. . . . . . . . . . . . . . . . . . . .

3.2 Fitted loss of the ULI waveguide shown in Figure $3.1 \ldots \ldots \ldots \ldots$

3.3 Simulated gaussian field distribution inside of an SMF-28 fiber. . . . . . . . . . . 21

3.4 Calculated index difference for an SMF-28 fiber (ThorLabs SMF-28-100). . . . . . 22

3.5 Mode intensity profile from a $40 \mu \mathrm{m}$ waveguide captured on an Electrophysics PV320 camera. . . . . . . . . . . . . . . . . . . . .

3.6 Calculated index difference obtained by Fourier optical beam propagation and the inverse Helmholtz technique. . . . . . . . . . . . . . . . . . . .

4.1 ULI inscription setup consisting of Aerotech air bearing stages, polarization control optics, and an IMRA ${ }^{\circledR}$ femtoseond laser. . . . . . . . . . . . . .

4.2 Microscope image taken perpendicular to the inscription direction. (Left) Inscription produced by varying translation speeds $(1 \mathrm{~mm} / \mathrm{s}-20 \mathrm{~mm} / \mathrm{s})$ and an inscription power of $100 \mathrm{~mW}$ (Right) Inscription produced by varying translation speeds $(1 \mathrm{~mm} / \mathrm{s}-20 \mathrm{~mm} / \mathrm{s})$ and an inscription power of $150 \mathrm{~mW} \ldots \ldots$ 
4.3 Longitudinal inscription geometry in which the waveguide is inscribed in the direction of the optical axis of the laser. The resulting structure has a symmetric cross-section due to the rotational symmetry of the inscription beam about the optic axis. The arrow indicates the direction of translation of the laser focus. . . . . . . .

4.4 Longitudinal inscription geometry in which the type III waveguide is inscribed in the direction of the optical axis of the laser. The resulting structure has a symmetric cross-section with guiding occurring within the modified region. The arrow indicates the direction of translation of the laser focus. The inset picture depicts the lateral translation of the beam for creating type III waveguides in the longitudinal

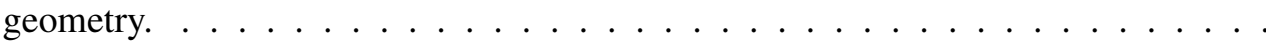

4.5 Transverse inscription geometry in which the waveguide is inscribed perpendicular to the direction of the optical axis of the laser. The resulting structure has a asymmetric cross-section due to the focusing dynamics of the lens used.The arrow indicates the direction of translation of the laser focus. . . . . . . . . . . .

4.6 Transverse inscription geometry in which the waveguide is inscribed perpendicular to the direction of the optical axis of the laser in a helical pattern. The resulting structure has a asymmetric cross-section due to the focusing dynamics of the lens used. The arrow indicates the direction of translation of the laser focus. . . . . . .

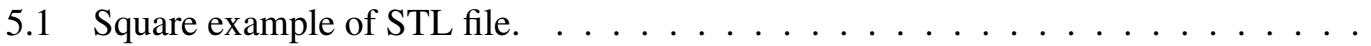

5.2 Four unique points of the triangles exported to the STL file version of Figure 5.1.

5.3 Square arch example for STL input. . . . . . . . . . . . .

5.4 Vertex information extracted from the STL file of Figure $5.3 \ldots \ldots \ldots$

5.5 Side-by-side of the model (right) and the extracted triangle verticies (left). . . . . 38

5.6 Modeled machine movements to create a 2 into 1 aperture waveguide structure. . .

5.7 Waveguide coupler from the machine movements in Figure 5.6. The picture shows the waveguide sample focused near the $7 \mathrm{~mm}$ depth. . . . . . . . . . .

5.8 Waveguide coupler from the machine movements in Figure 5.6. The picture shows the waveguide sample focused near the $0 \mathrm{~mm}$ depth. . . . . . . . . . . .

5.9 Propagating mode observed after propagating in the waveguides shown in Fig-

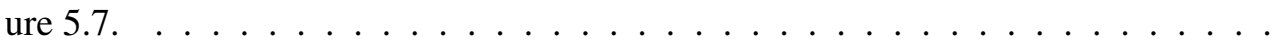


5.10 Waveguides inscribed in $\mathrm{ZnSe}$ with varying radii of curvature from $50 \mathrm{~mm}$ to $500 \mathrm{~mm}$. The inscription process fails to modify the $\mathrm{ZnSe}$ approximately halfway through the translation distance. The picture shows several attempts at creating curved waveguides. . . . . . . . . . . . . . . . .

5.11 Scanned electron microscope image of nanogratings formed at $65 \mu \mathrm{m}$ depth (sample cleaved and polished at writing depth) with polarization parallel (a) and perpendicular (b)to the scan direction. This picture has been reproduced from Bhardwaj et

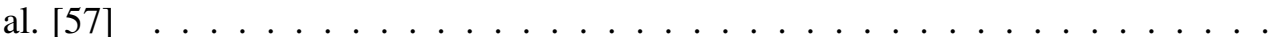

5.12 Waveguides inscribed in $\mathrm{ZnSe}$ with varying radii of curvature from $50 \mathrm{~mm}$ to $500 \mathrm{~mm}$. The polarization direction of the inscription beam was rotated to keep the polarization direction perpendicular to the propagation direction of the waveguide. 43

$5.1350 / 50$ waveguide splitters inscribed in a $10 \mathrm{~mm} \times 7 \mathrm{~mm}$ sample of $\mathrm{ZnSe} . \quad \ldots \quad . \quad 44$

5.14 Model of the 9 to 1 coupler created to scale in SketchUp. . . . . . . . . . . . . 45

5.15 Inscribed strcuture in ZnSe using the 9 to 1 coupler model from SketchUp. The waveguides show spurious modification at greater depths. . . . . . . . . . . .

6.1 Cladding structure created in $\mathrm{Cr}: \mathrm{ZnSe}(\mathrm{A})$ and $\mathrm{Fe}: \mathrm{ZnSe}(\mathrm{B})$ using the ULI setup described in the previous section. The waveguides had a diameter of approximately $110 \mu m$ (A) and $80 \mu m$ (B) respectively. Photo was taken looking into the direction of propagation. . . . . . . . . . . . . . . . .

6.2 Waveguides inscribed in Cr:ZnSe under non-optimal inscription parameters. The waveguides range in size from $40 \mu m$ to $120 \mu m \ldots \ldots$. . . . . . . .

6.3 Measured absorption cross-sections of Cr:ZnSe and Fe:ZnSe as a function of wavelength. The cross-section was calculated with the quoted sample concentration. . . 50

6.4 Cavity configurations for Fe:ZnSe and Cr:ZnSe waveguide lasers. . . . . . . . .

6.5 Calculation of the threshold pump power required for inversion in a material as a function of spot size. .................... 55

6.6 Facet damage that occurred during $\mathrm{CW}$ pumping of the $\mathrm{Cr}: \mathrm{ZnSe}$ waveguide. . . . . 56

6.7 Optical performance of the $111 \mu m, \mathrm{Cr}: \mathrm{ZnSe}$ waveguide laser. $\ldots \ldots \ldots \ldots$ 
6.9 Output spectrum of the Cr:ZnSe waveguide laser centered at $2522 \mathrm{~nm}$ measured with an Acton SP2500 spectrometer with a $0.5 \mathrm{~nm}$ resolution. . . . . . . . . . . . 58

6.8 A) Output mode profile of the ULI waveguide in Cr:ZnSe at an output power of $1 \mathrm{~W}$. The photo has been scaled and resized for viewing. B) The unscaled image of the beam propagating through the bulk crystal. The waveguide structure can be seen below the beam and indicated by the red arrow. . . . . . . . . . . . . . . .

6.10 Slope efficiency obtained from the Fe:ZnSe waveguide laser. Inset picture shows the output mode of the Fe:ZnSe waveguide laser. . . . . . . . . . . .

6.11 Output spectra from the Fe:ZnSe waveguide laser obtained using a Thorlabs OSA (Model OSA205). . . . . . . . . . . . . . . . .

6.12 Inscribed waveguide structure showing modified cladding and unmodified waveguide core. The picture was taken looking into the direction of propagation of the waveguide. The waveguide core was measured from the edges of the modified region. . .

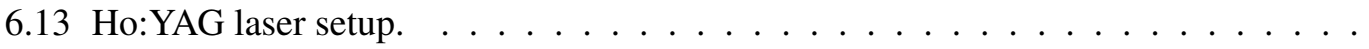

6.14 Experimental setup of the Cr:ZnSe pulsed waveguide laser, with cavity mirrors placed directly against the waveguide sample. . . . . . . . . . .

6.15 Normalized temporal output of the pump pulse(blue) and the signal output pulse(red). The pump and signal pulses were synchronized for comparison purposes. Unsynchronized pulses lead to a slight shift between pump and signal pulses due to the pulse buildup time of the resonator. However, the time shift between pump and signal pulses is small due to the short cavity length. . . . . . . . . . .

6.16 Slope efficiency of the Cr:ZnSe waveguide laser pumped by a pulsed Ho:YAG laser. The error bars represent pulse to pulse variations in the absorbed and output energies. 68

6.17 Output spectra of the Cr:ZnSe waveguide laser centered around $2492 \mathrm{~nm}$. . . . . .

$6.18 \mathrm{M}^{2}$ measurement of the pulsed waveguide output using 90/10 knife edge scan. . . .

6.19 A) Output mode shape measured by a Electrophysics PV320 camera after collimation and $30 \mathrm{~cm}$ of propagation. B) Image of the vertically translated waveguide structure showing the unguided pump beam . . . . . . . . . . . . .

6.20 Bragg reflector for reflecting a single wavelength from several wavelengths. . . . . 
6.21 Helical inscription of waveguide structures $\ldots \ldots \ldots \ldots \ldots$

6.22 Bulk sample of $\mathrm{Cr}: \mathrm{ZnSe}$ with Bragg reflectors on either side of the waveguide struc-

6.23 Example of Bragg structures inscribed in ZnSe. (a) Endface image of the helical waveguide (b) Top-down image of the helical waveguide . . . . . . . . . . . .

6.24 Experimental setup for testing of the Bragg structured waveguides. . . . . . . . .

6.25 Top: Theoretical reflectivity a waveguide Bragg grating with 40 evenly spaced, supported modes. Bottom: Output spectrum of identical waveguides with and without Bragg structuring. . . . . . . . . . . . . . . .

7.1 Cracking observed in Ho:YAG during initial testing of ULI parameters. The crack propagated laterally through all ULI structures, rendering the sample unusable. The picture was taken looking into the direction of propagation of the waveguide. . . .

7.2 Waveguides inscribed in a 0.5 at.\% Ho:YAG sample. The picture was taken looking into the direction of propagation of the waveguide. . . . . . . . . . . . .

7.3 Cavity configuration used for testing of the multimode waveguides. . . . . . . . . .

7.4 Laser performance for various output coupler reflectivities for the $80 \mu \mathrm{m}$ multimode

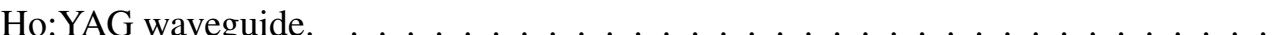

7.5 Spectral output of the $80 \mu \mathrm{m}$ waveguide dependent on output coupler reflectivity. .

7.6 Slope efficiency for the single mode waveguides. . . . . . . . . . . . .

7.7 $\mathrm{M}^{2}$ measurement for the multimode waveguide. The $1 / \mathrm{e}^{2}$ width was plotted as a function of distance when focused using a $10 \mathrm{~cm}$ focal length lens. Subset picture shows the far field output mode profile in a $250 \mu m \times 250 \mu m$ image. . . . . . . .

7.8 $\mathbf{M}^{2}$ measurement for the single mode waveguide. The $1 / e^{2}$ width was plotted as a function of distance when focused using a $10 \mathrm{~cm}$ focal length lens. Subset picture shows the far field output mode profile in a $120 \mu \mathrm{m} \times 120 \mu \mathrm{m}$ image. . . . . . . . 90

7.9 $\mathrm{Yb}^{3+} / \mathrm{Ho}^{3+}$ energy level diagram illustrating the pumping scheme of the co-doped material. The diagram has been reproduced from Rothacher et al. [109]. . . . . . . 91

7.10 Outward radial cracking of waveguides inscribed in the Yb:Ho:YAG sample. . . . 
7.11 Output power for in-band pumping of the Yb:Ho:YAG waveguide. . . . . . . .

7.12 Cavity configuration used for $940 \mathrm{~nm}$ pumping of the Yb:Ho:YAG waveguide sample. 94

7.13 Upconversion in the Yb:Ho:YAG sample pumped by $940 \mathrm{~nm}$ diodes. . . . . . . . 95

8.1 Basic picture of a 2-level laser band structure. . . . . . . . . . . . . . . . . . 97

8.2 Modeled output from a single pass amplifier using the rate equations set forth in Equation 8.5. The model predicted a maximum output of $0.78 \mathrm{~W}$ through a $1 \mathrm{~cm}$ amplifier with $200 \mathrm{~mW}$ of seed power and $1 \mathrm{~W}$ of pump power. . . . . . . . .

8.3 Round-trip gain of a simulated waveguide laser based on Cr:ZnSe. The iteration number represents the number of cavity round-trips. . . . . . . . . . . . . . . 100

8.4 Modeled slope efficiency for a Cr:ZnSe laser with a 70\% reflective outcoupler and no loss or thermal effects and the parameters from Table 8.1 . . . . . . . . . . . . 101

8.5 8th order polynomial fit to the experimental data of Deloach et al. [4]. . . . . . . . 104

8.6 (a) Geometry of a $40 \mu \mathrm{m}$ diameter waveguide structure $500 \mu \mathrm{m}$ away from the cooling surface at $y=0$. (b) Geometry of a $40 \mu \mathrm{m}$ diameter waveguide structure centered $20 \mu \mathrm{m}$ away from the cooling surface at $y=0 \ldots \ldots \ldots$. . . . . . 105

8.7 Modeled results for a $10 \mathrm{~W}$ heat source centered in a $40 \mu \mathrm{m}$ waveguide located $20 \mu \mathrm{m}$ from the cooling face . . . . . . . . . . . . . . . 106

8.8 Modeled results for a $10 \mathrm{~W}$ heat source centered in a $40 \mu \mathrm{m}$ waveguide located $500 \mu \mathrm{m}$ from the cooling face. . . . . . . . . . . . . . . . . 107

8.9 Calculated change in the threshold pump power as a function of input pump power. 108

8.10 Simulated temperature as a function of waveguide depth for Cr:ZnSe. $\quad \ldots \ldots$. . 108

8.11 Critical pump power required for the temperature change to decrease the excited state lifetime of the laser material. . . . . . . . . . . . . . . 110

8.12 Modeled results for a $10 \mathrm{~W}$ heat source centered in a $40 \mu \mathrm{m}$ waveguide located $20 \mu \mathrm{m}$ from the cryogenically cooled face. . . . . . . . . . . . . . . . . 111

8.13 Modeled results for a $10 \mathrm{~W}$ heat source centered in a $40 \mu \mathrm{m}$ waveguide located $500 \mu \mathrm{m}$ from the cryogenically cooled face. . . . . . . . . . . . . . . . . 112 
8.14 Critical pump power required for the temperature change to decrease the excited state lifetime of the laser material modeled for cryogenic cooling. . . . . . . . . . 113

8.15 6th order polynomial fit to the experimental data of Adams et al. . . . . . . . . . 113

8.16 Simulated temperature as a function of waveguide depth for Fe:ZnSe. . . . . . . 114

8.17 Critical power for Fe:ZnSe under cryogenic cooling. . . . . . . . . . . 115

A1 Generation of the first Stokes from KGW using a $2 \mu m$ pump laser. . . . . . . . 133

A2 First attempted femtosecond modification of GaP. . . . . . . . . . . . . . . . 134

A3 First attempted femtosecond modification of KGW . . . . . . . . . . . . 135 


\section{LIST OF TABLES}

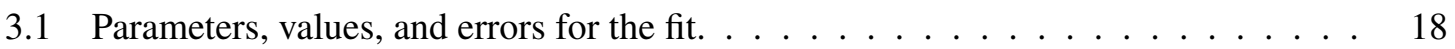

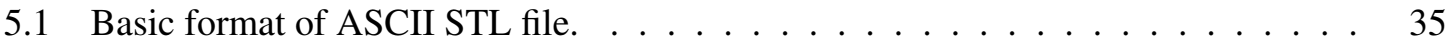

5.2 STL file for square shown in Figure $5.1 \ldots \ldots \ldots$

6.1 Summary of bulk laser power records $\ldots \ldots \ldots \ldots \ldots$. . . . . . . . 47

6.2 Summary of waveguide laser power records $\ldots \ldots \ldots \ldots$. . . . . . . . . 48

6.3 Parameters used for calculation of the threshold pump power. . . . . . . . . . . . . 54

6.4 Parameters used for inscription of Bragg structured cladding waveguides. . . . . . 75

7.1 Basic summary of inscription parameters and outcome of the inscription process in

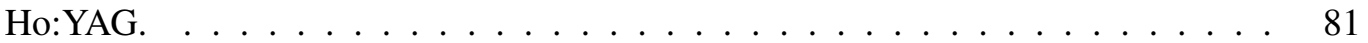

7.2 Slope efficiencies and maximum output powers of Figure 7.4 . . . . . . . . . . . . 84

7.3 Slope efficiencies and output powers of Figure $7.6 \ldots \ldots \ldots$. . . . . . . . 87

8.1 General parameters for testing the differential equations shown in Equation 8.5. . . 98

8.2 Modeled output power from ULI waveguides. . . . . . . . . . . . . . . . . 102

8.3 Output for various outcoupler reflectivities for Cr:ZnSe, Fe:ZnSe and Ho:YAG materials. Experimentally obtained output powers and slope efficiencies are included for comparison. . . . . . . . . . . . . . . . . . . . 102

8.4 Summary of relevant material parameters. . . . . . . . . . . . . . . . . 109 
8.5 Summary of modeling results for varying operating temperature and waveguide depth.116

A1 Raman material parameters . . . . . . . . . . . . . . . . . . . 130

A2 Material parameters of KGW . . . . . . . . . . . . . . . . . . 132 


\section{CHAPTER I}

\section{INTRODUCTION}

Since the advent of the laser in 1960 [1], constant advancements have been made in the field of lasers. Development of materials from ruby to Nd:YAG to Ti:Sapphire resulted in a wide range of wavelengths available for scientific applications. These laser sources provided the cornerstone for the development of laser sources extending into wavelength ranges $>1 \mu \mathrm{m}$. Wavelengths greater than $1 \mu \mathrm{m}$ are of extreme interest and importance to the scientific community due to two factors. First, there are a myriad of molecular absorption features in the $2-12 \mu m$ range. Second, the atmospheric transmission windows are in the $2-5 \mu m$ and $8-12 \mu m$ ranges. The existence of these features drives applications such as remote chemical sensing, laser communications, and medical applications.

Historically, the types of sources operating in the $2-12 \mu m$ range were gas lasers based on $\mathrm{CO}, \mathrm{CO}_{2}$, or He-Ne, operating at $4-8 \mu m, 8-12 \mu m$ and $3.39 \mu m$ respectively. Lead salt and raman shifted lasers were also widely used to generate Mid-IR radiation. Lead salt lasers have been demonstrated operating from $3-15 \mu m$ and with as much as $1 J$ of pulse energy, albeit with low efficiency [2]. Raman shifted lasers relying on the Raman shift from hydrogen or deuterium have generated radiation in the wavelength range of $3-5 \mu \mathrm{m}$.

In the last few decades, we have seen a dramatic increase in the variety of laser sources operating in the $2-5 \mu \mathrm{m}$ range. Sources such as optical parametric oscillators (OPOs), quantum cascade 
lasers (QCLs)[3], rare-earth lasers, and transition metal lasers have seen wide implementation across many applications. Each of these lasers have their own advantages and disadvantages. OPOs can operate over the entire $2-12 \mu \mathrm{m}$, however it is very difficult to get energetic pulses or continuous wave $(\mathrm{CW})$ operation due to the intensity dependence of nonlinear operation. Additionally, QCLs can achieve wavelength operation only at $>3 \mu \mathrm{m}$. Again, QCLs have the disadvantage of not being able to operate with energetic pulses due to the lack of energy storage capability in the device. QCLs can typically only operate on discrete transitions, thus they are not widely tunable. Rare-earth lasers can typically operate over a narrow wavelength range depending on the dopant utilized, crystal host, and doping concentration. Rare-earth lasers can also store larges amounts of energy, thus making them ideal for pulsed operation. Transition metal lasers operate in a unique range, where they have some energy storage capability, moderate tunability, and can operate in both a CW or a Q-Switched configuration.

Recent years have shown increased interest in development of $2-5 \mu \mathrm{m}$ laser sources. This interest is due to the atmospheric transmission window enabling a wide variety of military and medical applications. Most of these types of applications require devices with decreased size, weight and power (SWAP) requirements. Thus, most of the traditional gas or lead salt systems are precluded due to their size and power requirements. QCLs have shown potential, but they still have efficiency issues while operating at room temperature and with high average power/high pulse energy. Transition metal doped chalcogenides and rare-earth materials offer a viable solution to the shortcomings of their predecessors. First demonstrated by DeLoach et al. [4], transistion metal lasers have been widely demonstrated to operate in the $2-5 \mu \mathrm{m}$ range $[5,6,7,8]$ relying on chromium and iron dopants and $\mathrm{ZnSe}, \mathrm{ZnS}$ and CdTe hosts. 
The limiting factors with power scaling of transition metal doped chalcogenides is typically attributed to thermal effects. The relatively high thermo-optic coefficient of $\mathrm{ZnSe}, 7 \times 10^{-6} \mathrm{~K}^{-1}$, coupled with the decreased florescence efficiency due to increased phonon assisted transitions severely limits the maximum output of the laser. Exotic architectures based on spinning disk, $\mathrm{CO}_{2}$ cooled systems have been shown to help mitigate the thermal issues. Chromium based systems utilizing this type of architecture have demonstrated output powers of up to $139 \mathrm{~W}$ at $2.5 \mu \mathrm{m}$. Likewise, iron based systems have demonstrated $35 \mathrm{~W}$ under pulsed, non-collinear pumping configurations $[9,10]$.

Another method for decreasing thermal effects is to confine laser operation to a waveguide structure. Confinement of the optical field to a waveguide structure decreases sensitivity of the laser resonator to thermal lens destabilization induced by optical pumping of the gain medium. Given a material with a positive thermo-optic coefficient under optical pumping conditions, the core refractive index of the waveguide will always increase relative to the cladding. Thus, the waveguide will experience an increased confinement due to the induced thermal profile.

In addition, waveguide operation of a laser has several other benefits compared to bulk lasers. First, the transverse mode profile can be engineered to operate in a single mode or a multimode regime. Second, waveguides have minimized free space optics, which decreases sensitivity to thermal drift or vibration instabilities. Third, the optical mode of a waveguide can be confined over a much larger distance than inside of a bulk laser where the mode is dependent on the resonator mirrors. Finally, the pump and signal overlap is maintained across the entire waveguide where as bulk lasers are dependent on external focusing optics and cavity optics to maintain overlap. In general, applications that require high peak power are typically based on bulk lasers, while low threshold, high gain operation is typically based on waveguide technology. 
Since their inception in the 1970s, waveguides have been a vital tool in modern technology. As the basis for most modern communications systems, fiber/waveguides have become even more essential to the telecommunications industry. Corning was the first company to demonstrate optical fibers with losses of less than $20 \mathrm{~dB} / \mathrm{km}$ [11]. Since then, optical fiber losses have dropped dramatically, readily reaching values of $<0.2 \mathrm{~dB} / \mathrm{km}$, thus enabling efficient, long distance data transmission over fibers. In conjunction with fiber technology, integrated optical technology has paralleled the advancements of fibers. Fabrication of thin film or multilayer dielectric structures to create photonic devices was first demonstrated by P. K. Tien [12]. This demonstration lead to many advances including wavelength division multiplexing devices (WDM), interferometers, and arrayed waveguide devices [13]. Additionally, other techniques such as ion diffusion or proton exchange have been deployed as techniques for creating guided-wave structures [14, 15].

In recent years, a new technique has surfaced as a versatile tool for creating photonic structures. This technique is referred to as ultrafast laser inscription (ULI), which was first demonstrated by Davis et al. [16], and has emerged as powerful tool for creating waveguides and devices [17, 18]. The process of ULI relies on an ultrashort pulse focused inside a material. The high intensity of the pulsed beam induces nonlinear absorption processes, which transfers the pulse energy to the material lattice. With careful experimental control of the laser parameters, a permanent change in the refractive index of the material can be obtained. Early demonstrations of this technology relied on low repetition rate $(1-5 k H z)$ Ti:Sapphire lasers with pulse widths of $\approx 100 \mathrm{fs}$. The introduction of high power, high repetition rate mode-locked fiber lasers has enabled a dramatic growth in the ability of ULI to create low-loss waveguide structures [19]. Moreover, ULI allows creation of 3D structures [20,21, 22], allowing fabrication of spatially distinct waveguide structures. The versatility of ULI has allowed applications in the areas of teleconmmunications, astrophotonics, biology, and laser physics to be addressed [23, 24]. 
In particular, ULI has seen increased utility in the creation of compact laser devices. The first useful ULI laser devices were created in a co-doped Er:Yb glass. These devices marked the first demonstration of greater than unity gain and the first laser oscillations in a ULI sample [25, 26]. Demonstration of active devices as since been expanded to a variety of materials including YAG[27], glass, ZBLAN, ZnS, ZnSe[28], YLF, and tungstates. Recent advances have lead to increased power output from ULI waveguides of up to $5.1 \mathrm{~W}$ [29]. 


\section{CHAPTER II}

\section{BACKGROUND}

Ultrafast Laser Inscription (ULI) has previously been shown to be a viable technique for decreasing the size of TM lasers[30][31]. In order to understand how ultrashort pulses interact with a medium, it is necessary to understand how light propagates in the dielectric medium.

\subsection{Section 1: Ultrafast Laser Inscription}

The propagation of light in a non-magnetic medium is described by Maxwell's equations:

$$
\begin{array}{r}
\vec{\nabla} \times \vec{E}=-\frac{\partial \vec{B}}{\partial t}, \\
\vec{\nabla} \times \vec{H}=\frac{\partial \vec{D}}{\partial t}, \\
\vec{\nabla} \cdot \vec{H}=0, \\
\vec{\nabla} \cdot \vec{D}=0,
\end{array}
$$

where $\vec{E}$ is the electric field, $\vec{H}$ is the magnetic field, $\vec{D}$ is the electric flux and $\vec{B}$ is the magnetic flux. The displacement field satisfies the relation

$$
\vec{D}=\epsilon_{0} \vec{E}+\vec{P}
$$

where $\epsilon_{0}$ is the electric permittivity of free space and $\vec{P}$ is the polarization of light. The magnetic satisfies the constitutive relation

$$
\vec{B}=\mu_{0} \vec{H}
$$


where $\mu_{0}$ is the magnetic permeability of free space. These equations can be solved to produce the wave equation for light

$$
\vec{\nabla}^{2} \vec{E}+\frac{1}{c^{2}} \frac{\partial^{2} \vec{E}}{\partial t^{2}}=-\mu_{0} \frac{\partial^{2} \vec{P}}{\partial t^{2}} .
$$

In the above equation, $\mathrm{c}$ is the speed of light. Additionally, in the case of small electric fields, the polarization vector is linearly related to the electric field (E) by the first order polarization susceptibility tensor, $\chi^{(1)}$

$$
\vec{P}=\epsilon_{0} \chi^{(1)} \vec{E}
$$

However, in the presence of large electric fields, higher order nonlinearities can be excited, thus the polarization can be expanded to

$$
\vec{P}=\epsilon_{0}\left(\chi^{(1)} \vec{E}+\chi^{(2)} \vec{E}^{2}+\cdots+\chi^{(i)} \vec{E}^{i}\right),
$$

where $\chi^{(i)}$ is the ith order susceptibility. For centro-symmetric materials, the polarization can be simplified to

$$
\vec{P}=\epsilon_{0}\left(\chi^{(1)} \vec{E}+\frac{3}{4} \chi^{(3)} \vec{E}^{2}\right) \vec{E}
$$

Substituting back into the original wave equation, the simplified wave equation is

$$
\vec{\nabla}^{2} \vec{E}+\frac{n^{2}}{c^{2}} \frac{\partial^{2} \vec{E}}{\partial t^{2}}=0
$$

where the refractive index, $\mathrm{n}$, is defined as

$$
n=\sqrt{1+\chi^{(1)}+\frac{3}{4} \chi^{3}|\vec{E}|^{2}}
$$

Noting that $|\vec{E}|^{2}=I$, the refractive index can be approximated as $n \approx n_{0}+n_{2} I$. Thus, if a short pulse on the order of picoseconds to femtoseconds is incident on the material, the nonlinear refractive index becomes crucial to beam propagation dynamics inside of the material. Assuming that the 
magnitude of $n_{2}$ is positive, a Gaussian beam propagating through the medium will experience a higher index in the center of the beam as compared to the edges of the beam leading to self-focusing. Self-focusing eventually focuses the beam to a small enough spot to reach peak intensities in the range of $T W / \mathrm{cm}^{2}$. Intensities in this range readily induce a strong nonlinear absorption, which transfers energy to the material lattice causing a permanent change in the local refractive index. Multiphoton absorption follows closely after the nonlinear absorption, which relies on an electron in the valence band absorbing multiple photons. The total photon energy must satisfy $m h \nu>E_{g}$, where $\mathrm{m}$ is the number of photons, $h$ is Plank's constant, $\nu$ is the frequency of light and $E_{g}$ is the bandgap energy of the material. At high laser intensity and low frequency, nonlinear ionization can lead to tunneling between conduction and valence bands. The strong field distorts the band structure and reduces the potential barrier between the valence and conduction bands[32]. Direct band to band transitions may then proceed by quantum tunneling of the electron from the valence to conduction band, which follows the Keldysh parameter

$$
\gamma=\frac{\omega}{e} \sqrt{\frac{m_{e} c n \epsilon_{0} E_{g}}{I}}
$$

The Keldysh parameter can also be used to describe multiphoton absorption and tunneling photoionization. In Equation 2.10, $\omega$ is the laser frequency, $I$ is the laser intensity, $m_{e}$ is the effective electron mass, $e$ is the charge of the electron, $c$ is the speed of light, $n$ is the refractive index, and $\epsilon_{0}$ is the permittivity of free space. If $\gamma$ is much greater than 1.5 , tunneling ionization dominates. For $\gamma \approx 1.5$, photoionization is a combination of tunneling and multiphoton ionization. For waveguide fabrication in dielectrics, $\gamma \approx 1$, so that nonlinear ionization is a combination of both tunneling ionization and multiphoton ionization [33]. The plasma generated by the photo-ionization causes the beam to defocus. Again, the self-focusing effect cause the beam to collapse back down to a point. The balance of focusing and defocusing leads to filamentation inside of the material[33]. An example of filamentation inside of a bulk medium can be seen in Figure 2.1. Depending on the interaction of 


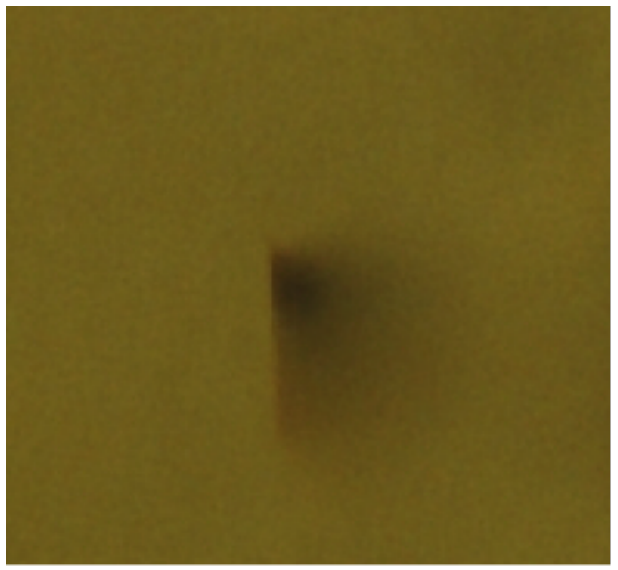

Figure 2.1: Femtosecond laser modification inside of a bulk $\mathrm{ZnSe}$ crystal. The modification was created by a $700 f s$ pulse focused with a 0.65 NA microscope objective.

the incident pulse, either positive or negative index changes can be achieved. This process has been shown by numerous authors to be a viable technique for creating waveguide structures[16, 19, 20]. However, ULI has only recently been demonstrated to be a useful technique for creating Mid-IR waveguide devices[34]. The modification of a material is typically divided into 3 types; type I, type II, and type III modification. These different modification types are shown in Figure 2.2. Type I (Figure 2.2 A) modification relies on a positive index change at the focus of the inscription laser. The positive change in index allows guiding in the region of laser damage providing the modification is not highly scattering. Type II (Figure $2.2 \mathrm{~B}$ ) modification relies on two damage regions to stress the material. The stress region induces a positive index change, which allows light to be guided in the stress region between the damage spots. Finally, type III (Figure $2.2 \mathrm{C}$ ) modifications rely on a symmetric type II modification to create a cladding structure. Each of these types of waveguides has benefits and disadvantages. Type I waveguides typically produce near single mode operation with small mode diameters $(<10 \mu m)$. Type II waveguides are typically polarization sensitive but are limited to small guided mode regions due to the small cross-sectional area where the stress induced refractive index change affects the guided light. Type III waveguides can be scaled to large 

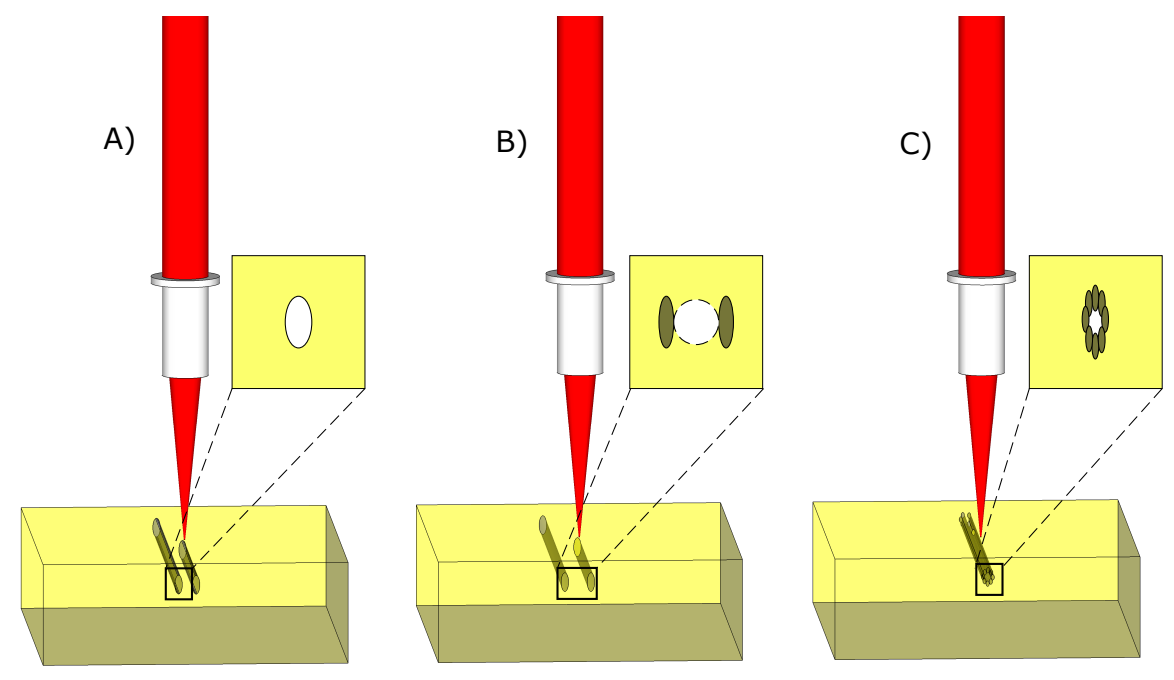

Figure 2.2: A) Type 1 modification B) Type II modification C)Type III modification

mode area waveguides. However, type III waveguides are not usually polarization sensitive except in cases of strong stress induced birefringence, uniaxial crystals or biaxial crystals.

\subsection{Physical Processes}

With a basic physical understanding of the beam propagation dynamics, the physical processes undergone during the inscription process can be explored. Due to the non-destructive/non-ablating nature of femtosecond laser modification, no mass or momentum changes occur. Thus, the material modification process can be described by the coupled energy equations for electrons and phonons in the two temperature approximation [35]

$$
\begin{gathered}
C_{e} n_{e} \frac{\partial T_{e}}{\partial t}=Q_{a b s}-C_{e} n_{e} \nu_{e n}\left(T_{e}-T_{L}\right)+\vec{\nabla} \cdot \vec{q}_{e l}, \\
C_{L} n_{a} \frac{\partial T_{L}}{\partial t}=C_{e} n_{e} \nu_{e n}\left(T_{e}-T_{L}\right)=\vec{\nabla} \cdot \vec{q}_{\text {phonon }},
\end{gathered}
$$


where $C_{e}$, is the electon specific heat, $C_{L}$ is the lattice specific heat, $n_{e}$ is the number density of free carrier electrons, $n_{a}$ is the number density of atoms, $Q_{a b s}$ is the absorbed energy density rate, $\vec{\nabla} \cdot \vec{q}_{e l}$ is the electron heat conduction flux, $\vec{\nabla} \cdot \vec{q}_{\text {phonon }}$ is the phonon conduction flux, $v_{e n}$ is the electron phonon energy exchange rate, and $F$ is the fluence from the laser pulse. For femtosecond pulses, the pulse interaction is much shorter than the electron-phonon energy exchange rate and the heat conduction time. If we consider a pulse where the electron-phonon energy exchange time is less than the pulse width, which is less than the heat conduction time, $\tau_{e-L}<\tau_{p}<\tau_{t h}$. Under these conditions, we can assume that the electron temperature and lattice temperature are at equilibrium. Thus, the electron conservation law can be rewritten as:

$$
\begin{gathered}
C_{e} n_{e} \frac{\partial T_{e}}{\partial t}=Q_{a b s}+\vec{\nabla} \cdot \vec{q}_{e l}, \\
C_{L} n_{a} \frac{\partial T_{L}}{\partial t}=\vec{\nabla} \cdot \vec{q}_{\text {phonon }} .
\end{gathered}
$$

Assuming that the absorbed photon rate is equal to the absorbed energy density rate, heat loss is negligible and that the electron/lattice temperature equalizes during the pulse interaction time, we can obtain the energy conservation law

$$
\begin{array}{r}
C_{e} n_{e} \frac{\partial T_{e}}{\partial t}=Q_{a b s}+\vec{\nabla} \cdot \vec{q}_{e l}, \\
C_{L} n_{a} \frac{\partial T_{L}}{\partial t}=\frac{\omega}{|1+\sqrt{\epsilon}|^{2}} I(r, z, t), \\
=\frac{2 A}{l_{a b s}} I(r, z, t) .
\end{array}
$$

The absorption coefficient, $A$, is defined by the Fresnel formula

$$
A=1-R=\frac{4 n}{(n+1)^{2}+k^{2}}
$$


The electron conservation law can then be solved for the temperature

$$
\begin{array}{r}
\frac{\partial T}{\partial t}=\frac{2 A}{l_{a b s}} I(r, z, t), \\
\int \frac{\partial T}{\partial t} d t=\int \frac{2 A}{l_{a b s} C_{L} n_{a}} I(r, z, t), \\
T=\frac{2 A}{l_{a b s} C_{L} n_{a}} \int I(r, z, t), \\
T=\frac{2 A F}{l_{a b s} C_{L} n_{a}} .
\end{array}
$$

This is the maximum temperature at in the focal region. We can now plot the temperature as a function of input pulse energy for ZnSe. For this calculation we will assume an absorption length of $l_{a b s}=10 \mu \mathrm{m}$, pulse energies from $200 \mathrm{~nJ}$ to $15 \mu \mathrm{J}$, a density of $n_{a}=5.42 E 6 \mathrm{~g} / \mathrm{m}^{3}$, lattice specific heat of $0.356 \mathrm{~J} / \mathrm{gC}$ and an absorption of 0.82 .

Assuming these values, temperatures well in excess of $1100{ }^{\circ} \mathrm{C}$ can be obtained. The rise in this temperature difference also induces a pressure wave inside of the material. A rough estimate of the internal pressure cause by the differential heating can be approximated by

$$
\sigma=E \alpha \Delta T
$$

where $\sigma$ is the stress due to heating, $E$ is the modulus of elasticity, $\alpha$ is the coefficient of thermal expansion and $\Delta T$ is the change in temperature. Assuming that $\alpha=7.1 \times 10^{-6} /{ }^{\circ} C, E=67.2 \mathrm{GPa}$ and $\Delta T=727{ }^{\circ} \mathrm{C}$, we can obtain a stress due to differential heating of $0.34 \mathrm{GPa}(\approx 50,000$ PSI). Under these conditions, several possibilities exist for what happens to the material. First, the material may undergo a phase change. For instance, $\mathrm{ZnSe}$ will undergo a phase change at from the zincblende crystal structure to the wurtzite structure at approximately $1400 K$ [36]. This temperature is easily attained at the focal spot of the ULI process. Second, the material experiences extreme pressure due to the large, fast temperature change. Pressures on the order of 30,000 psi can produce large shifts in the band-gap of the material of up to $1000 \mathrm{~cm}^{-1}$ [37]. Pressures and temperatures in this range have shown to be highly useful in post-processing of laser samples in order to obtain higher spectral brightness and remove material defects[38]. 


\subsection{Transition Metal Lasers}

Transition metal lasers fill a unique role in the laser community. Transition metals have only partially filled $d$ electron orbitals. When the transition metal ions are placed into a crystal field, they experience vibronically broadened transitions leading to large emission bandwidth from the material. Most commonly, transition metals are doped into II-VI compounds such as $\mathrm{ZnS}, \mathrm{ZnSe}$, CdSe, and CdTe. However, the most common transition metal laser is actually Ti:Sapphire, which can emit over the $650-1100 \mathrm{~nm}$ range [39]. Transisition metal lasers based on $3 d$ transisiton are highly sensitive to the host crystal field. The sensitivity of these ions arises from electron-phonon interactions due to the unshielded outer electons. In comparison, rare earth ions have completely filled $4 d, 5 s, 5 p$, and $6 s$ shells and rely on valence electrons entering either the $4 f$ or $5 d$ shells [40]. The most common rare earth ions used for laser media are neodynium, thulium, ytterbium, holmium, and erbium. A comparison of the energy levels in transisition metals and rare earths are shown in Figure 2.3,

Transition metals located within a tetrahedrial crystal field have two electronic levels, which originate from the crystal field splitting of the ${ }^{5} D$ ground state of the free ion with the $d^{4}$ configura-

tion [42]. The two levels, ${ }^{5} T_{2} \longrightarrow{ }^{5} E$, which produces broad absorption and emission features due to vibrational broadening and Jahn-Teller splitting of the allowed energy levels [42]. Depending on the crystal host, the emission and absorption features can span $>1 \mu m$ and have peak emission cross sections centered throughout the $2-5 \mu m$ range [43]. It should be noted that some transition metals, such as $\mathrm{Fe}^{2+}$ experience inverted levels such that the ${ }^{5} E$ level is above the ${ }^{5} T_{2}$ level [44]. 


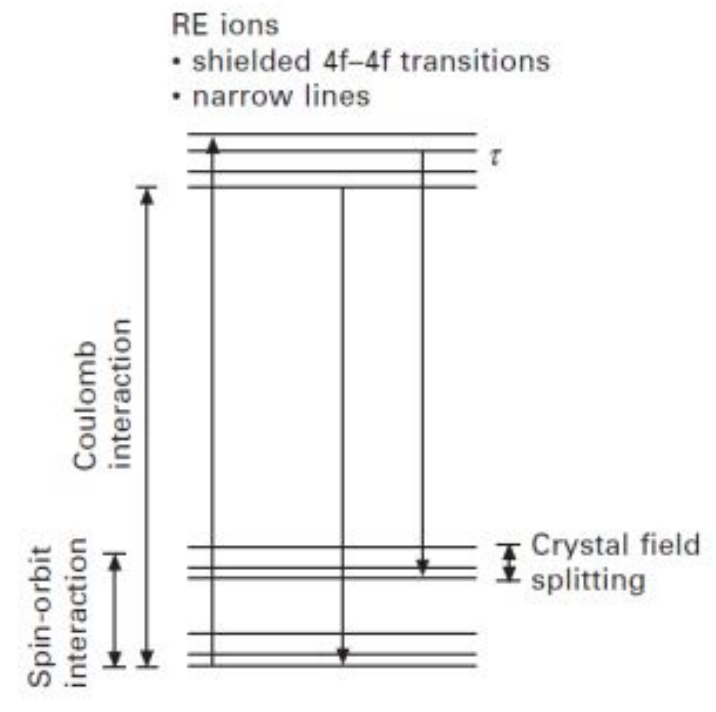

(a)

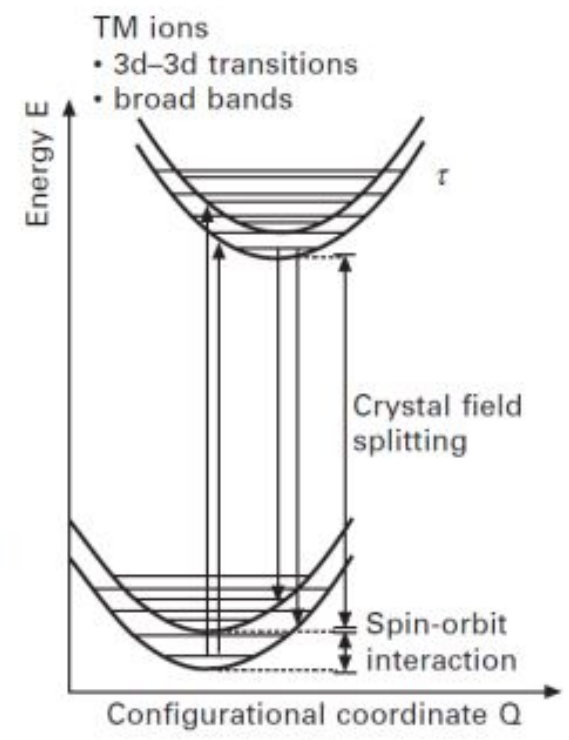

(b)

Figure 2.3: Energy level diagrams showing transitions from rare earth (RE) ions and transition metal (TM) ions shown in (a) and (b) respectively. This diagram has been reproduced from Petermann et al. [41] 


\section{CHAPTER III}

\section{CHARACTERIZATION TECHNIQUES}

Complete test and evaluation of ULI waveguides is essential to analysis of the ULI performance. When a structure is created with ULI, it is necessary to measure two important characteristics. First, the propagation losses of the waveguide structure. Second, the index profile of the waveguide or the index difference from the core to the cladding. The propagation losses affect the usability of the waveguide. If the propagation losses of the waveguide are too high, the device will not guide enough light to be useful for many applications. Additionally, the index profile provides the confinement for the optical beam. The index structure and index difference play an important role in engineering the coupling in to and out of the waveguides. The importance of these ULI features requires a set of characterization techniques to be set forth. The techniques will focus on characterizing the optical losses and index structure of the ULI waveguides.

\subsection{Losses}

Image analysis of waveguide losses is a nondestructive technique for estimating losses from a waveguide structure [45]. The image analysis technique eliminates the need to carefully factor out entrance and exit coupling losses required for input/output measurements. Scattered intensity at any location along the beam path in a sample will be proportional to the beam intensity, I, at that point, assuming the losses are isotropic in nature. The beam intensity at that point will be determined by 
all propagation losses (scattering and absorption) experienced by the beam up to that point. The light imaged by the camera will be proportional to the scattered and fluorescing light (assuming both are within the camera detection band) and both decay spatially with the same combined loss coefficient. The loss of beam intensity at position $\mathrm{x}$ is given by

$$
\frac{d I(x)}{d x}=-\left(\alpha_{S}+\alpha_{A}\right) I(x)
$$

where $\alpha_{S}$ is a scattering coefficient and $\alpha_{A}$ is an absorption coefficient. We will assume that the loss coefficients are constant along the propagation path. It should be noted that if there are variations in the inscription quality along the propagation length of the ULI waveguide, which can occasionally happen, the loss coefficient will likely be larger than expected. The solution to the above differential equation is

$$
I(x)=I_{0} e^{-\left(\alpha_{S}+\alpha_{A}\right) x},
$$

where $I_{0}$ is the intensity at some starting point in the sample and not at the surface to avoid surface scattering. The scattered intensity at any point is proportional to $\alpha_{S} I(x)$ but it will decay exponentially as the combined absorption and scattering loss coefficients, $\alpha_{T}$

$$
\alpha_{T}=\alpha_{A}+\alpha_{S}
$$

It should be noted that for accurate measurement of the propagation loss, a test wavelength that is not absorbed by the sample should be used. An image analysis process was developed using MATLAB for converting an image of the scattered light from a waveguide, Figure 3.1. The pixel data from Figure 3.1 consists of a matrix with values ranging from 0 to 255 ( 8 bits). The scattering is not uniform across the transverse direction of the waveguide so it is convenient to sum the columns into a single intensity value (i.e. for a single $\mathrm{x}$ value). The image was then cropped down to just the scatter from the waveguide. Figure 3.1 was fit to

$$
I(x)=a e^{\left(\frac{-x}{\tau}\right)}-y .
$$




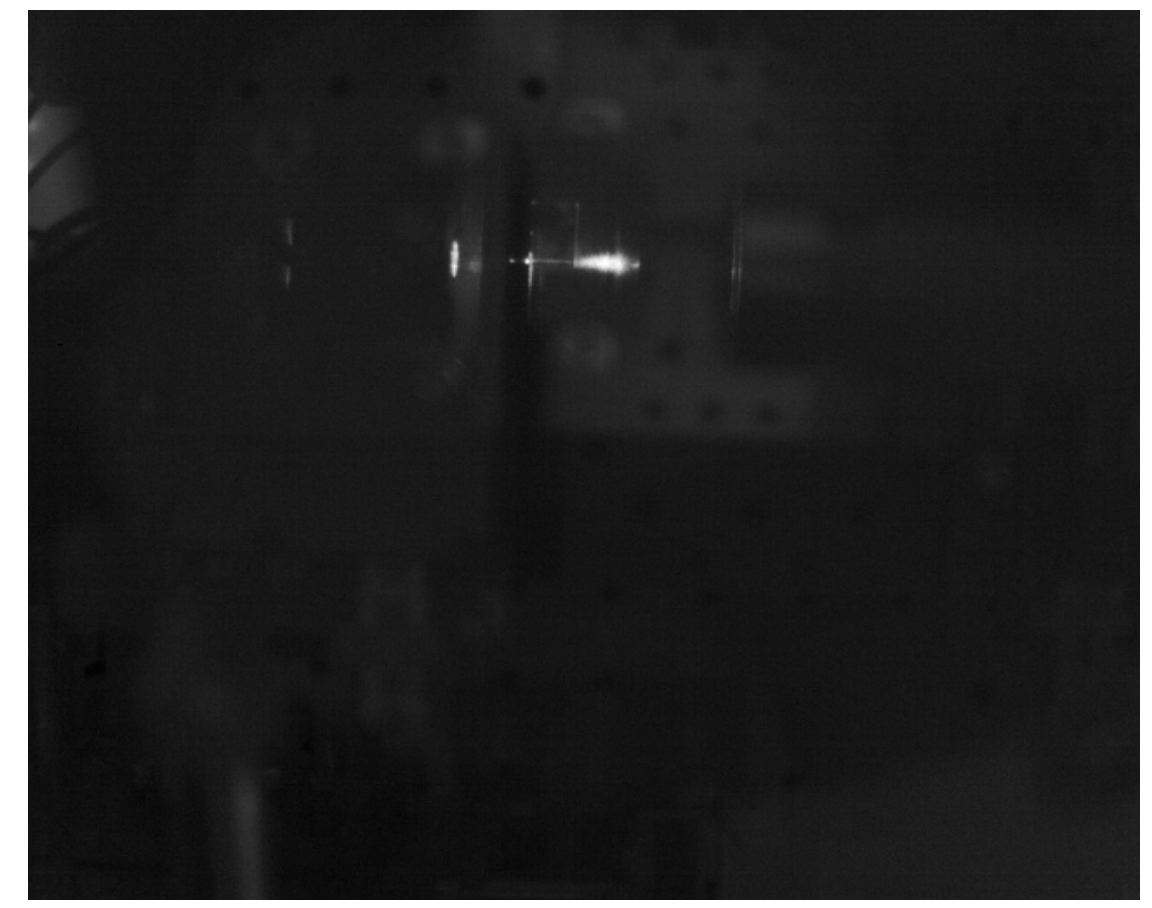

Figure 3.1: Scattered intensity image from a ULI waveguide in ZnSe captured using a FLIR SC6700 thermal camera.

The loss of the waveguide is then related to $\frac{1}{-\tau}$ multiplied by a calibration factor related to the experimental setup. The calibration factor, $C$, is the resolution of the image in $\mu \mathrm{m} /$ pixel. The fit values, along with the physical dimensions of the sample and the camera pixels, can be seen in Table 3.1.

This technique was the primary tool for measuring losses in the ULI waveguides.

\subsection{Index Difference}

\subsubsection{Direct Measurement}

One of the most straight-forward techniques for measuring the index structure of a guided-wave structure is to measure the divergence of the beam exiting the waveguide. From the definition of 


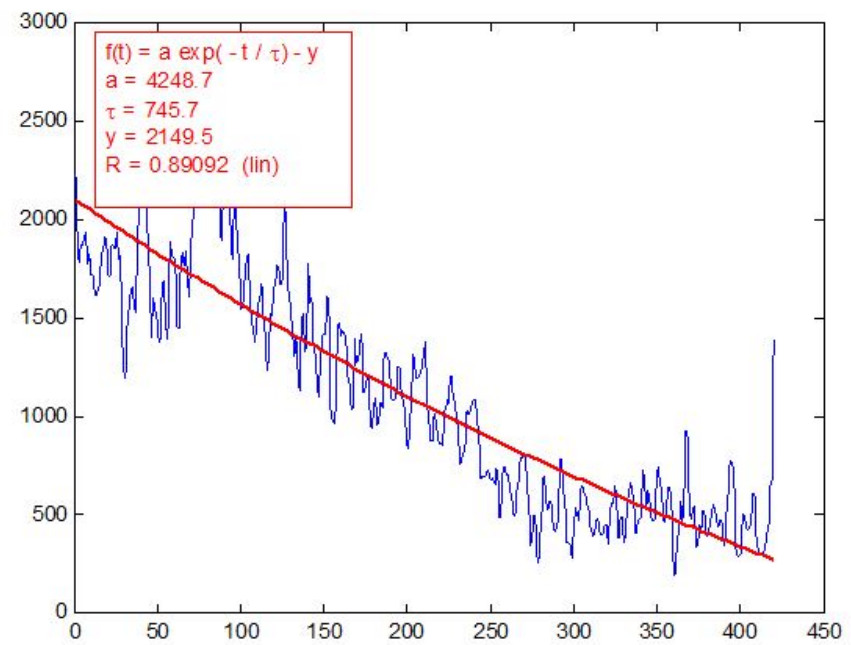

Figure 3.2: Fitted loss of the ULI waveguide shown in Figure 3.1

Table 3.1: Parameters, values, and errors for the fit.

\begin{tabular}{|l|c|r|}
\hline Parameter & Value & Error \\
\hline$y$ & 2149.5 & $10 \%$ \\
a & 4248.7 & $19 \%$ \\
$\tau$ & 745.7 & $14 \%$ \\
Crystal Length (L) & $5.413 \mathrm{~mm} / 429$ pixels & $0.1 \% / 3 \%$ \\
Pixel Calibration (C) & $12.6 \mu \mathrm{m} /$ pixel & $4 \%$ \\
Loss $\alpha=1 /(\tau C)$ & $0.106 \mathrm{~mm}^{-1}(4.6 \mathrm{~dB} / \mathrm{cm})$ & $18 \%$ \\
\hline
\end{tabular}


the numerical aperture for a step-index fiber, Equation 3.5, the divergence angle is directly related to the index difference between the core and cladding

$$
N A=\sin (\theta)=\sqrt{n_{\text {core }}^{2}-n_{\text {clad }}^{2}}
$$

Equation 3.5 can be solved for the cladding index, Equation 3.6. If it is assumed that the core index remains unmodified and that the ULI process lowers the surrounding index, Equation 3.6 can be solved by directly measuring the divergence of the beam from the waveguide

$$
n_{\text {clad }}=\sqrt{n_{\text {core }}^{2}-(\sin (\theta))^{2}} .
$$

Figure 3.1 shows the propagation of light within a ULI waveguide, as well as the divergence of the light from the waveguide. In Figure 3.1, the diverging beam is only observed because the sample was set on a mount, which was longer than the waveguide. The light exiting the waveguide scattered from the mount allowing a direct measurement of the divergence angle. Figure 3.1 was loaded into MATLAB to allow software measurement of the full divergence angle, which was measure to be $43^{\circ}$. Using the Sellmeier equations for $\mathrm{ZnSe}[46]$, a bulk index of 2.44 calculated at a wavelength of $1908 \mathrm{~nm}$. Utilizing these values, an index difference of approximately 0.03 was obtained. It should be noted that the index difference in ULI waveguides does not necessarily follow a stepindex configuration. However, direct measurement does provide an upper estimate to the induced index difference from ULI.

\subsubsection{Inverse Helmholtz}

In addition to losses from a waveguide, it is desirable to know the index profile of the inscribed waveguide to determine the guiding properties. In order to determine the index profile, we can either directly measure the divergence of the beam from the end facet of the waveguide or back-calculate the index profile from the near field beam profile. Direct measurement of the waveguide output 
tends to produce large uncertainties in the index profile depending on the measured values. For instance, the difference in the index of refraction between a beam diverging with a full angle of $26^{\circ}$ and $27^{\circ}$ (waveguide core is assumed to have an index of 2.4) is approximately 0.001 . Additionally, direct measurement also assumes that the waveguide structure is completely symmetric, which is not always true. A more valuable technique for measuring the index profile is the Inverse Helmholtz technique. From Maxwell's equations, we know

$$
\nabla^{2} \vec{E}+\frac{n^{2}}{c^{2}} \frac{\partial^{2} \vec{E}}{\partial t^{2}}=0
$$

Provided that the beam is guided by an index profile, we can simplify Equation 3.7 to

$$
\nabla_{t r}^{2} \vec{E}+\left(k^{2} n^{2}-\beta^{2}\right) \vec{E}=0
$$

where $k$ is the wavevector of the light, $n$ is the index profile and $\beta$ is the propagation constant of the guided wave. Equation 3.8 can be rearranged to find:

$$
n^{2}=\frac{\beta^{2}}{k^{2}}-\frac{1}{k^{2}} \frac{\nabla_{t r}^{2} \vec{E}}{\vec{E}}
$$

Additionally, from waveguide theory we note that $\frac{\beta^{2}}{k^{2}}=n_{\text {eff }}^{2}$, which is the effective index of the guided wave. Using this relation and rearranging Equation 3.9, we can obtain

$$
n^{2}=n_{e f f}^{2}-\frac{\lambda^{2}}{4 \pi^{2}} \frac{\nabla_{t r}^{2} \vec{E}}{\vec{E}}
$$

Equation 3.10 is of interest because it relates the electric field to the index structure. It should also be noted that the index profile and the electric field are 2 variable functions (i.e. $E=E(x, y)$ and $n=n(x, y)$ ). With modern computational methods, calculating the transverse Laplacian of a 2 dimensional matrix is straight forward. For reference, the Laplacian is defined as Equation 3.11.

$$
\nabla_{t r}^{2} \vec{E}=\frac{\partial^{2} \vec{E}}{\partial x^{2}}+\frac{\partial^{2} \vec{E}}{\partial y^{2}}
$$




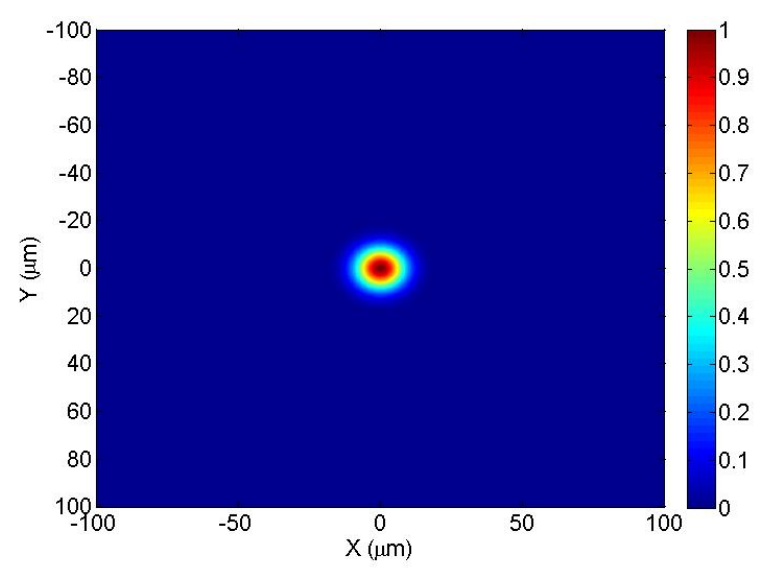

Figure 3.3: Simulated gaussian field distribution inside of an SMF-28 fiber.

The Laplacian can be represented as a finite difference formulation:

$$
\begin{array}{r}
\frac{\partial^{2} \vec{E}}{\partial x^{2}}+\frac{\partial^{2} \vec{E}}{\partial y^{2}}= \\
\Delta x^{2} \vec{E}(x, y)
\end{array}
$$

This formulation is what will be implemented for solving the index profile. This approach can be used to calculate the index difference for a known fiber. Assume an SMF-28 fiber (ThorLabs Model SMF-28-100). The core has an index of 1.452 and a cladding index of 1.447 yielding an index difference of 0.005 . Because the properties of SMF-28 fibers are well known, we know that the mode field diameter is approximately $10.4 \mu \mathrm{m}\left(1 / \mathrm{e}^{2}\right)$ for $1550 \mathrm{~nm}$ light. The lowest order mode guided by the fiber is approximately Gaussian, thus we can model it as a 2-D Gaussian profile, Figure 3.3. Applying the above equations to the mode profile shown in Figure 3.3, we can obtain a plot of the index profile of the SMF-28 fiber, Figure 3.4. From this figure, it can be seen that the index difference from the core to the cladding is 0.006 . This approximately corresponds to the index difference in an SMF-28 fiber, which is 0.005 . However, the index profile obtained is not usually precise, but more useful is the index difference calculated by the technique. Due to the second order 


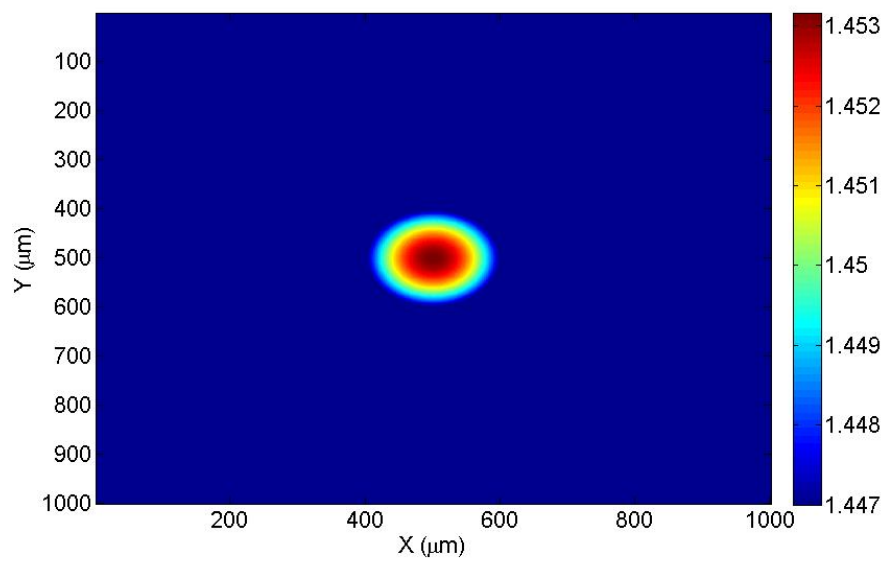

Figure 3.4: Calculated index difference for an SMF-28 fiber (ThorLabs SMF-28-100).

differential in Equation 3.10, the refractive index profile index profile obtained using the inverse Helmholtz technique is highly sensitive to noise on the measured data. In addition, the technique also requires careful windowing and prevention of sharp changes in the field profile. Even so, the technique has been successfully applied to ultrafast laser inscribed waveguides [47].

With verification that the inverse Helmholtz technique works, it can be applied to the ULI waveguides in this work. The far-field profile of the beam was captured using an Electrophysics PV-320 camera, Figure 3.5. 


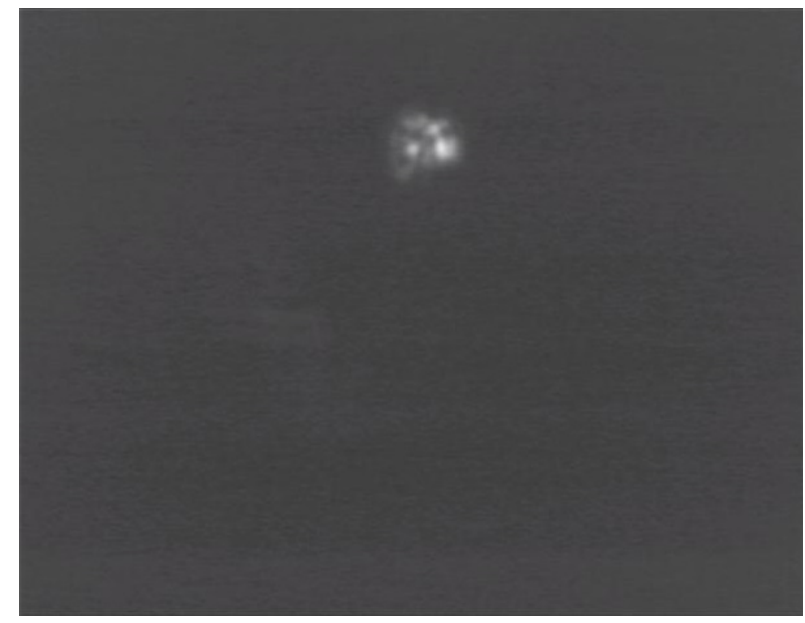

Figure 3.5: Mode intensity profile from a $40 \mu m$ waveguide captured on an Electrophysics PV-320 camera.

Assuming that the far field phase front of the beam was flat, the beam was back propagated to the waveguide facet using Fourier beam propagation[48]. The same numerical method as previously demonstrated was utilized to calculate the index difference using the near-field mode profile. The resultant index difference can be seen in Figure 3.6. From Figure 3.6, it can be seen that the maximum index difference obtained was 0.02 The usefulness of this index structure is debatable. The sensitivity of this technique to noise, sharp changes, and numerical windowing makes it unreliable for precise calculation of the index profile. However, it does provide a useful tool for verifying the accuracy of the beam divergence measurement, which is in close agreement with the calculated index difference of 0.02. Additionally, a phase retrieval algorithm could be utilized to improve the accuracy of this measurement [49]. 


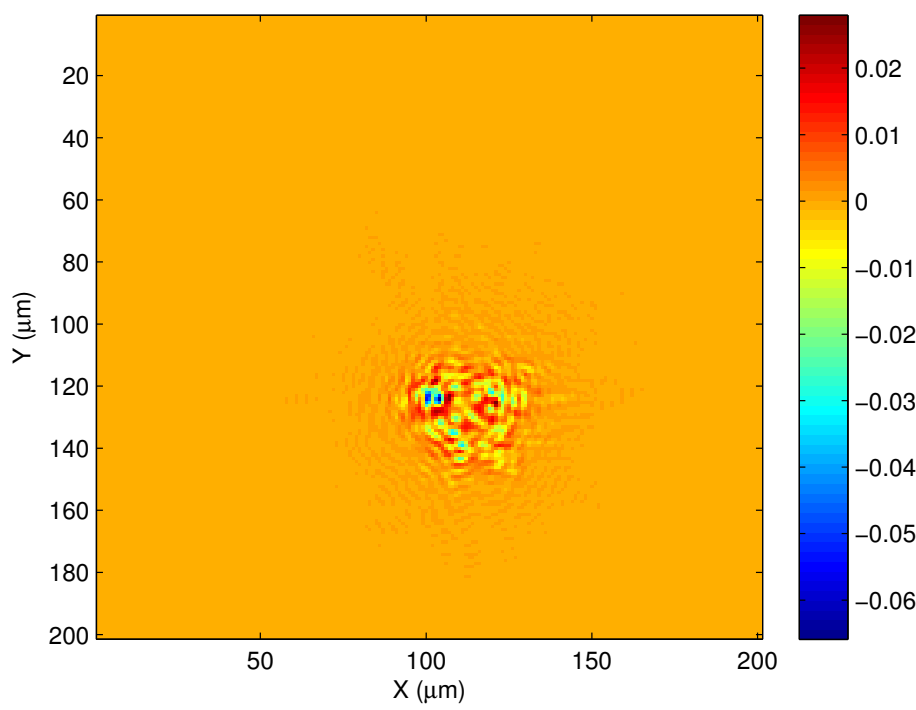

Figure 3.6: Calculated index difference obtained by Fourier optical beam propagation and the inverse Helmholtz technique. 


\section{CHAPTER IV}

\section{INSCRIPTION}

Ultrafast laser inscription is dependent upon a variety of parameters that influence the physical processes induced inside of the material. These parameters are pulse energy, pulse repetition frequency, pulse width, focal spot size, polarization, wavelength, inscription velocity and phase front tilt. These parameters will be discussed in order. The pulse energy of the inscription laser contributes to the total amount of energy transferred from the laser pulse to the material. Typically, higher pulse energies lead to stronger material modification. Of course, this only applies to energy ranges that are non-destructive (i.e. energies that do not ablate or dissociate molecules within the material). The actual mechanisms that give rise to the index change inside the material are not well understood, but the change could arrise from changes in the degree of crystallinity of the material. The pulse repetition frequency mainly contributes to any thermal effects in the material [50]. High repetition rates induce more thermal accumulation in the sample due to multi-pulse interaction. Multi-pulse interactions occur when the energy from the preceding pulse has not completely dissipated from the focal volume of the beam. Conversely, low repetition rates allow for more thermalization time between pulses, minimizing thermal effects. The pulse width of the inscription laser has a multitude of effects on the nature of material modification. Nejadmalyeri et al. have discussed the role of pulse width on the resulting structures in lithium niobate[51], where pulses $<850 f s$ produced high propagation losses with no or little guiding. The focus spot of the inscription laser 
affects the focal spot of the beam, which controls the peak intensity of the laser. The intensity of the beam is the main driving force behind nonlinear self-focusing inside the sample. The polarization of the inscription laser has been shown to affect many aspects of ULI waveguides from propagation losses [51] to form birefrigence [52]. For wide bandgap materials, such as semiconductors and dielectrics, the wavelength of the inscription laser determines the multiphoton absorption process, tunneling ionization, or avalanche ionization. Inscription velocity, similar to the pulse repetition frequency, affects the cumulative interaction of adjacent pulses. Careful control of pulse repetition frequency, writing speed and pulse energy has been shown to effectively control precipitation of crystalline materials from a glass host $[53,54]$. Finally, the phase front tilt of the beam has been shown to generate a "quill" effect, which is a directionally dependent modification arising from anisotropic trapping of the electron plasma by a tilted front of the ultrashort laser pulse.

Accounting for the wide variety of control parameters necessary to inscription, a ULI setup was designed with the help of Heriot Watt University. The system, pictured in Figure 4.1, was based on a regeneratively amplified Yb:fiber laser from IMRA ${ }^{\circledR}$. The repetition rate of the laser is adjustable from $100 \mathrm{kHz}$ up to $5 \mathrm{MHz}$ with an average output power of over $1.3 \mathrm{~W}$ for all repetition rates. The laser also used an adjustable pulse, which allows control of the pulse chirp on the laser output. The pulse compressor allowed the system to elongate or compress the temporal pulse of them system. Using this adjustable compressor it is possible to achieve pulse durations ranging from $350 \mathrm{fs}$ to $1.5 \mathrm{ps}$.

In addition, a high precision translation stage is necessary for ULI. The stage must be capable of fast, precise movements with no spring-back. The stages chosen for this were Aerotech ${ }^{\circledR}$ air bearing stages (model: ABL2000 and AVL100). These stages were capable of $200 \mathrm{~mm}$ of lateral movement and $25 \mathrm{~mm}$ of vertical movement. The stages were capable of movement accuracy of $200 \mathrm{~nm}$ with translation velocities of up to $100 \mathrm{~mm} / \mathrm{s}$. 


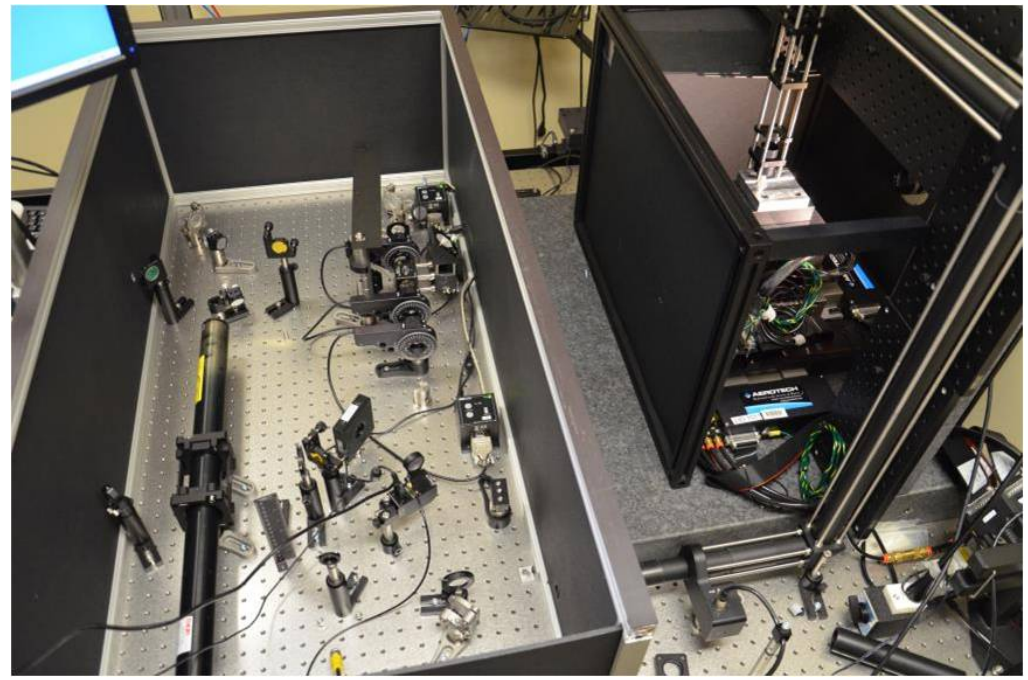

Figure 4.1: ULI inscription setup consisting of Aerotech air bearing stages, polarization control optics, and an IMRA ${ }^{\circledR}$ femtoseond laser.

\subsection{Femtosecond Laser Inscription}

II-VI semiconductors have a plethora of applications from laser devices [4] to solar cells [55]. $\mathrm{ZnSe}$, in particular, offers high transmission throughout the entire $2-12 \mu \mathrm{m}$ range. II-VI semiconductors can also exhibit high optical nonlinearity $\left(\chi^{(2)}\right)$, which unitary semiconductors lack due to their centrosymmetric crystal structure. The versatility of ZnSe makes it an ideal host for developing ULI waveguide structures. In order to fabricate waveguides in ZnSe using ULI, a variation of parameters is needed in order to determine the best parameters for modifying $\mathrm{ZnSe}$. Referring to previously published values for inscription in $\mathrm{ZnSe}[28]$, several power levels, scan rates and pulse widths were determined to provide the best starting point for successful index modification of ZnSe. The power levels ranged from $50 \mathrm{~mW}$ to $300 \mathrm{~mW}$, the scan rates ranged from $0.5 \mathrm{~mm} / \mathrm{s}$ to $25 \mathrm{~mm} / \mathrm{s}$, and the pulse widths were varied from $350 \mathrm{fs}$ to $1 \mathrm{ps}$. These values were tested by focusing the inscription laser into a sample of ZnSe using a $0.68 \mathrm{NA}, 3.1 \mathrm{~mm}$ focal length aspheric 
lens, which focused to a spot size of approximately $1 \mu \mathrm{m}$. The resulting modification from the the $100 \mathrm{~mW}$ and $150 \mathrm{~mW}$ scans can be seen in Figure 4.2.

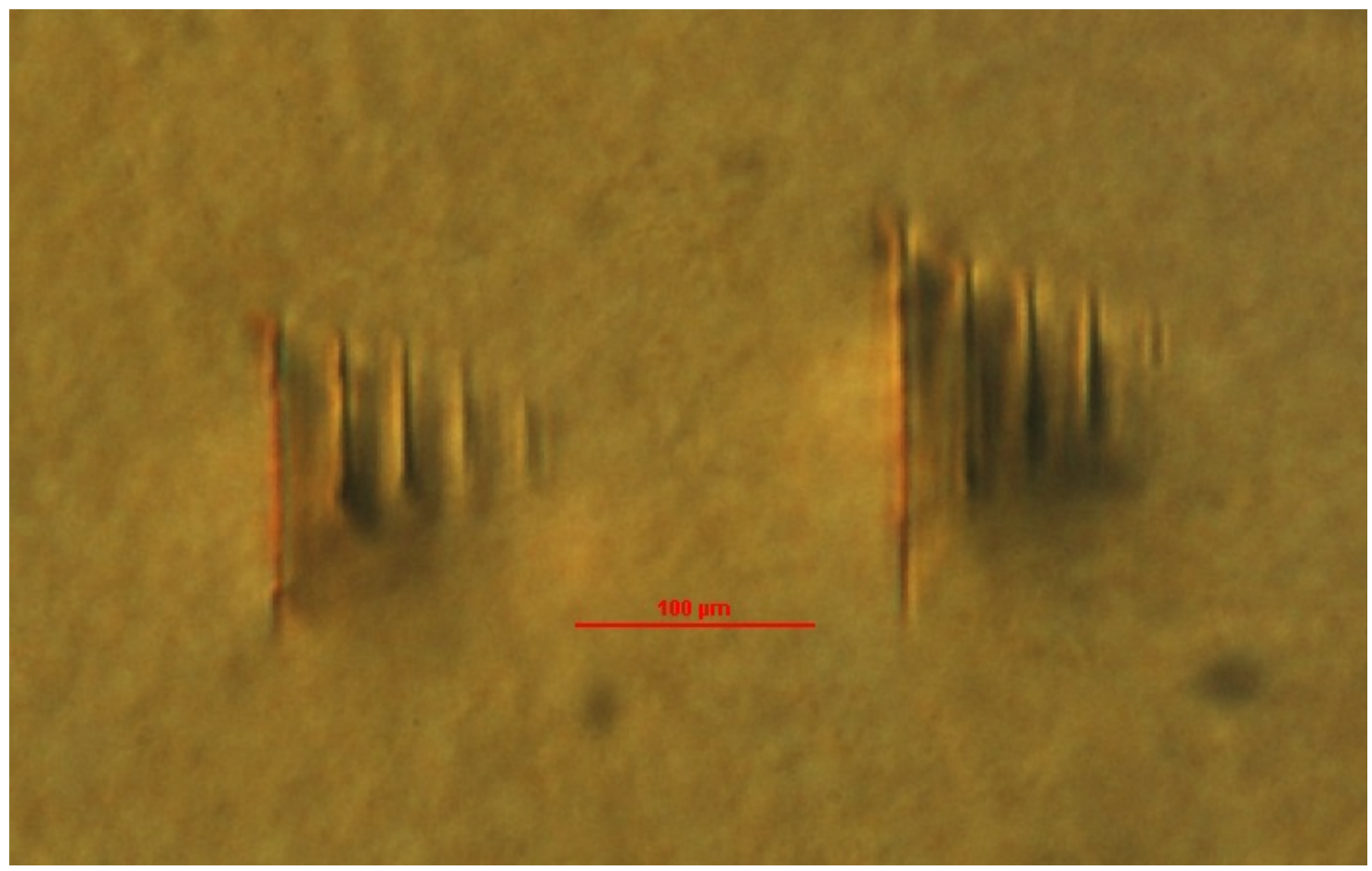

Figure 4.2: Microscope image taken perpendicular to the inscription direction. (Left) Inscription produced by varying translation speeds $(1 \mathrm{~mm} / \mathrm{s}-20 \mathrm{~mm} / \mathrm{s})$ and an inscription power of $100 \mathrm{~mW}$ (Right) Inscription produced by varying translation speeds $(1 \mathrm{~mm} / \mathrm{s}-20 \mathrm{~mm} / \mathrm{s})$ and an inscription power of $150 \mathrm{~mW}$

In Figure 4.2, a 1 ps pulse was used for both sets of features. Each set contains modifications from translating at speeds of $1 \mathrm{~mm} / \mathrm{s}, 2 \mathrm{~mm} / \mathrm{s}, 5 \mathrm{~mm} / \mathrm{s}, 10 \mathrm{~mm} / \mathrm{s}$, and $20 \mathrm{~mm} / \mathrm{s}$. The longest filamentation region corresponds to the slowest translation speed. In general, the slower the translation speed used for ULI, the longer the filamentation. The length of the filamentation region is directly related to the self-focusing of the pulse and successive pulses add to the filamentation length. 


\subsection{Geometries for Inscription}

The direction of writing, focusing dynamics and desired index structure all affect the geometry required for ULI. There are two main geometries that have been demonstrated for creating ULI waveguides.

\subsubsection{Longitudinal Geometry}

In this arrangement, the sample and stage movement is in the direction of the optical axis of the inscription beam, Figure 4.3. This provides a symmetrical beam cross-section along the optic axis and can result in a symmetric waveguide structure given the proper inscription parameters. However, the length over which waveguides can be inscribed is limited to the working distance of the lens used. As ULI generally employs high NA lenses with short focal lengths, this severely limits the achievable length of the resultant structures. 


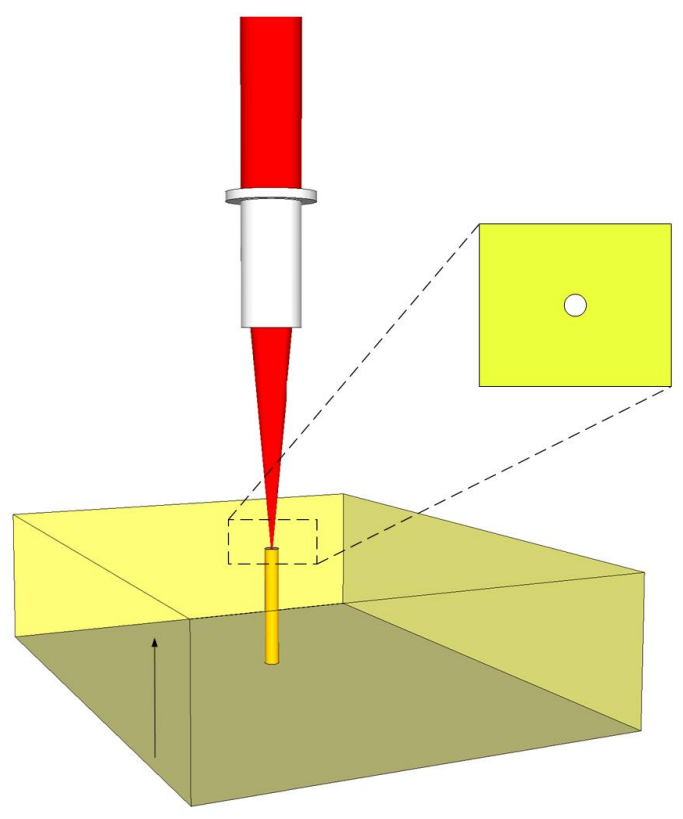

Figure 4.3: Longitudinal inscription geometry in which the waveguide is inscribed in the direction of the optical axis of the laser. The resulting structure has a symmetric cross-section due to the rotational symmetry of the inscription beam about the optic axis. The arrow indicates the direction of translation of the laser focus.

This configuration can be used for type II or type III waveguides as well. The only modification is that instead of translating in the vertical direction, the focus needs to be translated in a circle while translating vertically, Figure 4.4. It should be noted that the translations requirements for the stage (i.e. stage precision) should be much less that the size of the designed feature size of the inscription process. 


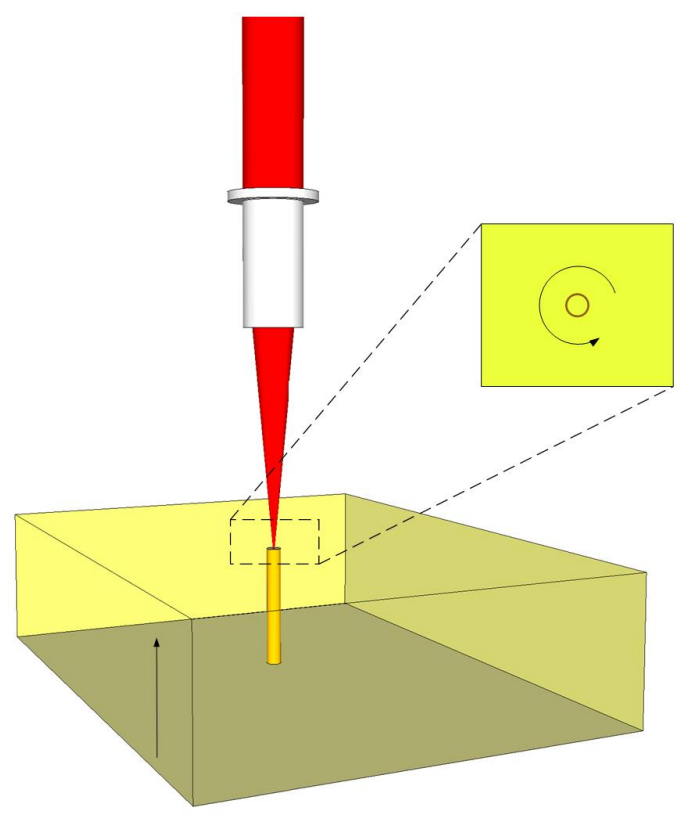

Figure 4.4: Longitudinal inscription geometry in which the type III waveguide is inscribed in the direction of the optical axis of the laser. The resulting structure has a symmetric cross-section with guiding occurring within the modified region. The arrow indicates the direction of translation of the laser focus. The inset picture depicts the lateral translation of the beam for creating type III waveguides in the longitudinal geometry.

\subsubsection{Transverse Geometry}

In the transverse geometry, the sample is translated perpendicularly to the optic axis of the laser beam, Figure 4.6. Transverse geometry overcomes the restriction in achievable length in the longitudinal geometry as the sample can be translated over arbitrary distances. However, this geometry results asymmetric cross-sections. For the transverse geometry, the intensity distribution of the focal volume is elongated through self-focusing in the material, in addition to critical modification over the Rayleigh range of the focusing lens. The modification asymmetry can be mitigated by utilization of a high NA lens due to the decrease in asymmetry for large numerical apertures. However, using a high NA lens severely limits the usable modification depth. 


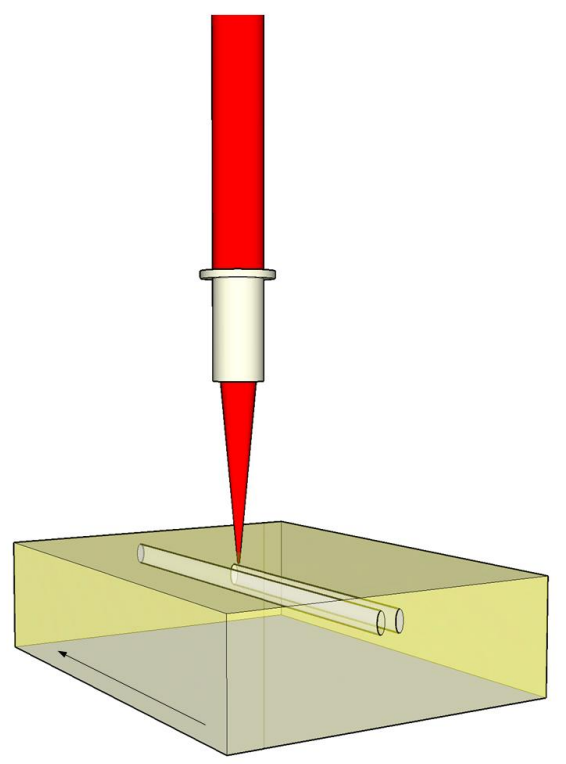

Figure 4.5: Transverse inscription geometry in which the waveguide is inscribed perpendicular to the direction of the optical axis of the laser. The resulting structure has a asymmetric cross-section due to the focusing dynamics of the lens used.The arrow indicates the direction of translation of the laser focus.

This configuration can also be used for type II or type III waveguide. Additionally, helical waveguides can also be employed with the transverse geometry to create smooth modification regions instead of several filamentation regions inscribed in a circular structure.. 


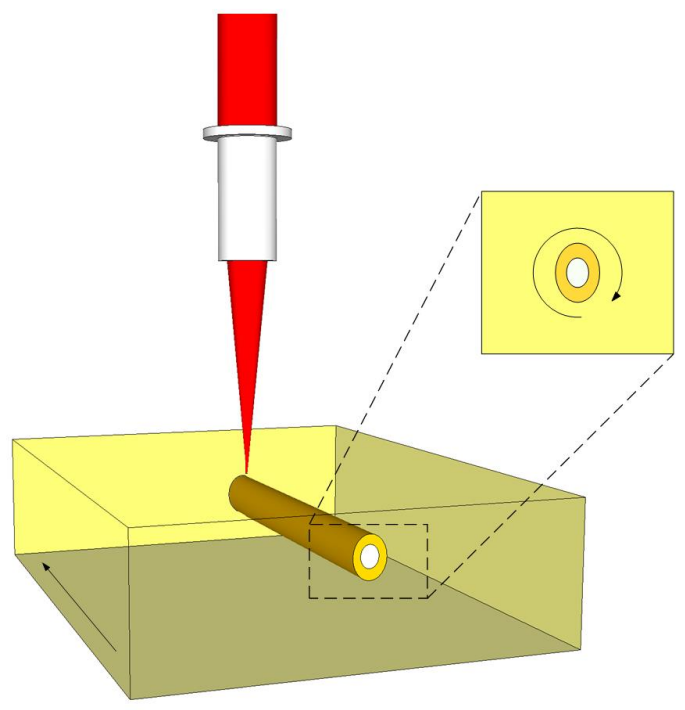

Figure 4.6: Transverse inscription geometry in which the waveguide is inscribed perpendicular to the direction of the optical axis of the laser in a helical pattern. The resulting structure has a asymmetric cross-section due to the focusing dynamics of the lens used. The arrow indicates the direction of translation of the laser focus. 


\section{CHAPTER V}

\section{GEOMETRY GENERATION}

A key process in creating waveguide structures with Ultrafast Laser Inscription (ULI) is creating the code to produce the desired waveguide structure. Typically, there are two ways of creating such code. First, the stage movements used for creating the waveguide structure can be hard coded by means of a script that programmatically generates G-code movements. G-code is the language used by the translation stage. This method becomes complicated when creating complex 3-D structures such as couplers, splitters or mode-strippers. The second method for creating structures is employed in several commercial pieces of software, which 3-D printers use. This method relies on CAD (Computer Aided Design) software to create the desired structure. From the CAD software, a STL (STereoLithography) file can be exported. The STL file is an unsctructured triangulated surface created by the unit normal of the triangle and the vertices of the triangle. The vertices are ordered by the right-hand rule using a three dimensional Cartesian coordinate system. In order for this information to be useful, an interpreting program must be written in order to extract the useful information from the STL file. One small caveat is that STL files come in two varieties; ASCII and Binary. Only ASCII type files will be considered in this work.

The goal of this work is to create a method for interpreting STL files into a G-code friendly format. Fortunately, ASCII STL files are exported in ASCII format, which can easily be imported into MATLAB. Once imported into MATLAB, the file is sorted by columns. The basic layout of an 
Table 5.1: Basic format of ASCII STL file.

\begin{tabular}{|c|}
\hline $\begin{array}{l}\text { facet normal } n_{i} n_{j} n_{k} \\
\text { outer loop } \\
\text { vertex } v_{1 x} v_{1 y} v_{1 z} \\
\text { vertex } v_{2 x} v_{2 y} v_{2 z} \\
\text { vertex } v_{3 x} v_{3 y} v_{3 z} \\
\text { end loop } \\
\text { end facet }\end{array}$ \\
\hline
\end{tabular}

STL file is shown below, Table 5.1. From this, we can see the facet normal vector $\left(n_{i} n_{j} n_{k}\right)$ and the location of the vertices of the triangle $\left(v_{x} v_{y} v_{z}\right)$. This process is looped over the entire file. Utilizing MATLAB, vertices and the facet normals can be extracted from the STL file.

To illustrate this process, assume a square as shown in Figure 1. The STL file for this shape can be exported, which is shown in Table 2.

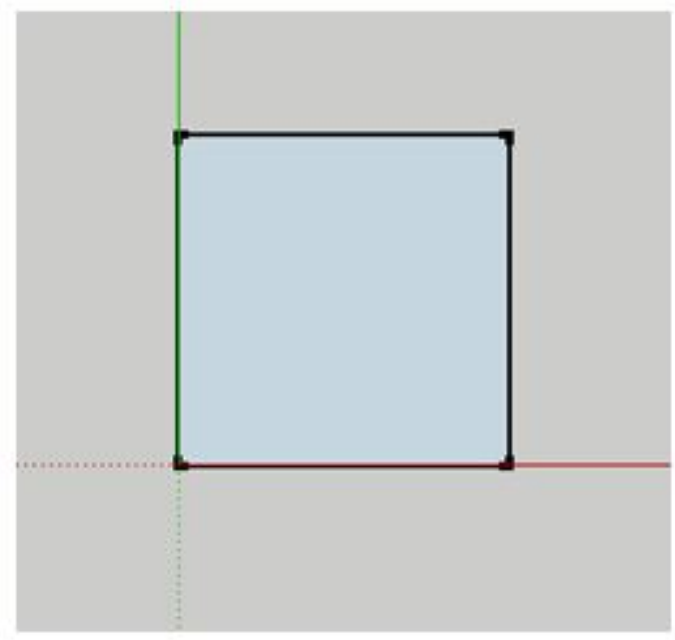

Figure 5.1: Square example of STL file. 
Table 5.2: STL file for square shown in Figure 5.1.

solid model
facet normal $0.00 .0-1.0$
outer loop
$\quad$ vertex 897.5897 .50 .0
vertex 0.00 .00 .0
vertex 0.0897 .50 .0
endloop
endfacet
facet normal $0.00 .0-1.0$
outer loop
$\quad$ vertex 0.00 .00 .0
vertex 897.5897 .50 .0
vertex 897.50 .00 .0
endloop
endfacet
end solid model

The algorithm developed in MATLAB can be used to decipher the STL file into vertices and facet normals. For the simple case of a square, it can be seen that the square can be divided into two triangles. However, two points of these triangles will be duplicates. In order to get rid of duplicates, the unique function in MATLAB to eliminate duplicate points. Elimination of duplicate points produces the plot of the 4 unique points of the two triangles, Figure 5.2. 


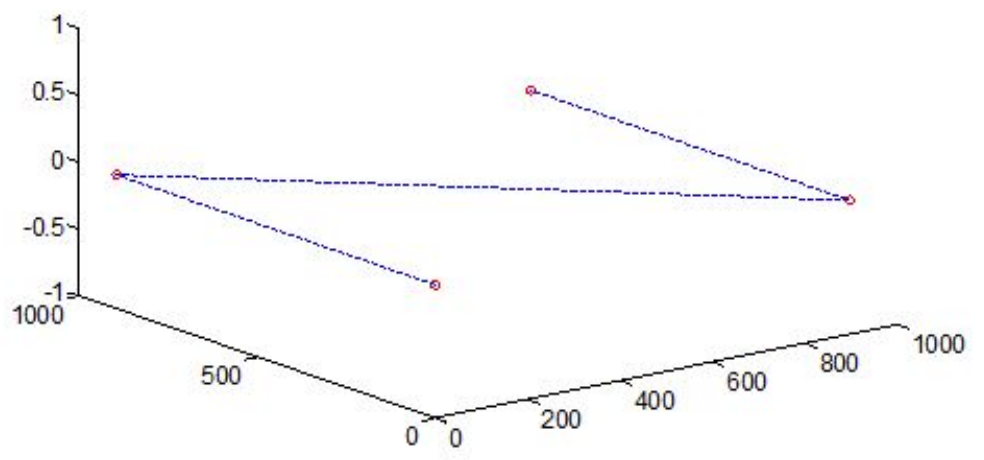

Figure 5.2: Four unique points of the triangles exported to the STL file version of Figure 5.1.

This basic technique can now be used to create more complicated structures in MATLAB using STL files. For example, let us consider the square arch shown in Figure 5.3. The STL file can be exported file from the CAD software into MATLAB to obtain the vertices of the model, Figure 5.4.

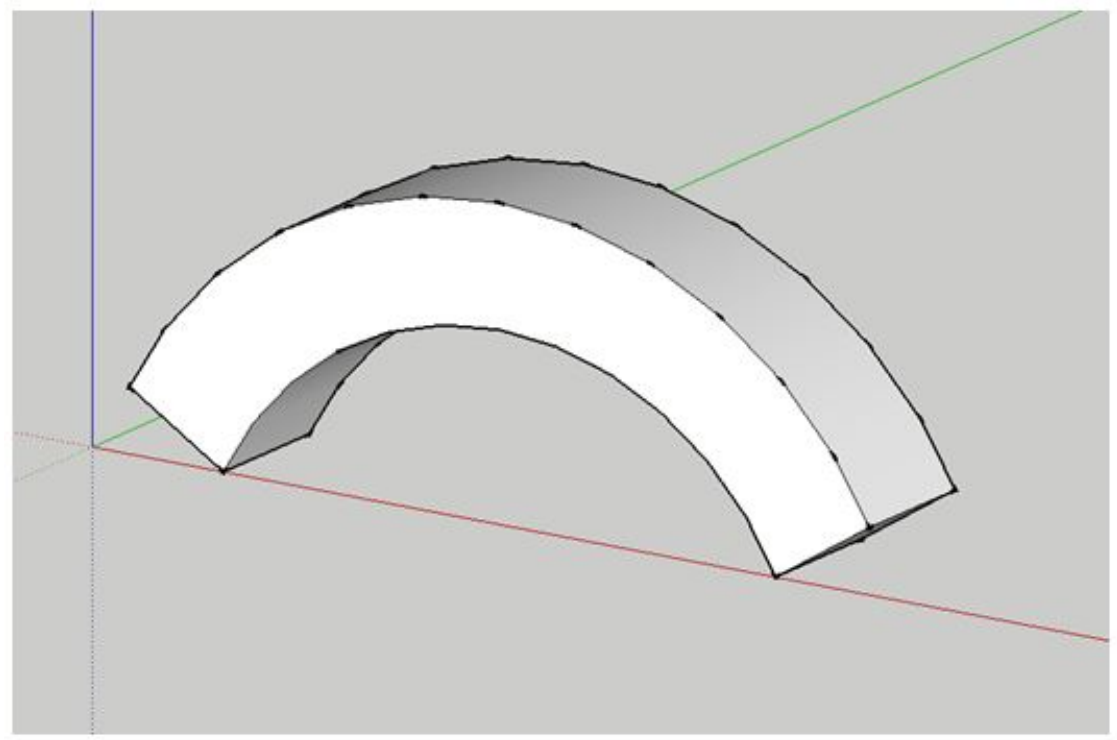

Figure 5.3: Square arch example for STL input. 


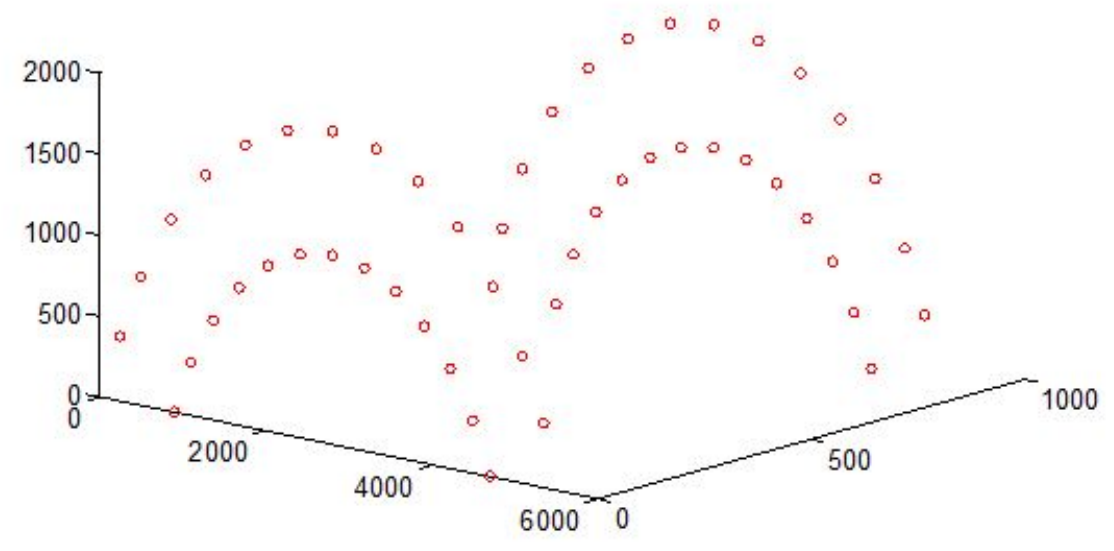

Figure 5.4: Vertex information extracted from the STL file of Figure 5.3.

This technique can be used for even more complex structures. Figure 5.5 shows a model of a linear array coupler in the CAD program and the extracted vertices from the STL file. The next step in this process is being able to sort and order the points in a logical order, which will allow direct translation to position movements in G-code. However, this method becomes very difficult if the CAD drawing contains all of the waveguide structures. The most realistic solution is to export the data from the $\mathrm{CAD}$ program in an ordered scheme (i.e. export data for a single waveguide at a time).
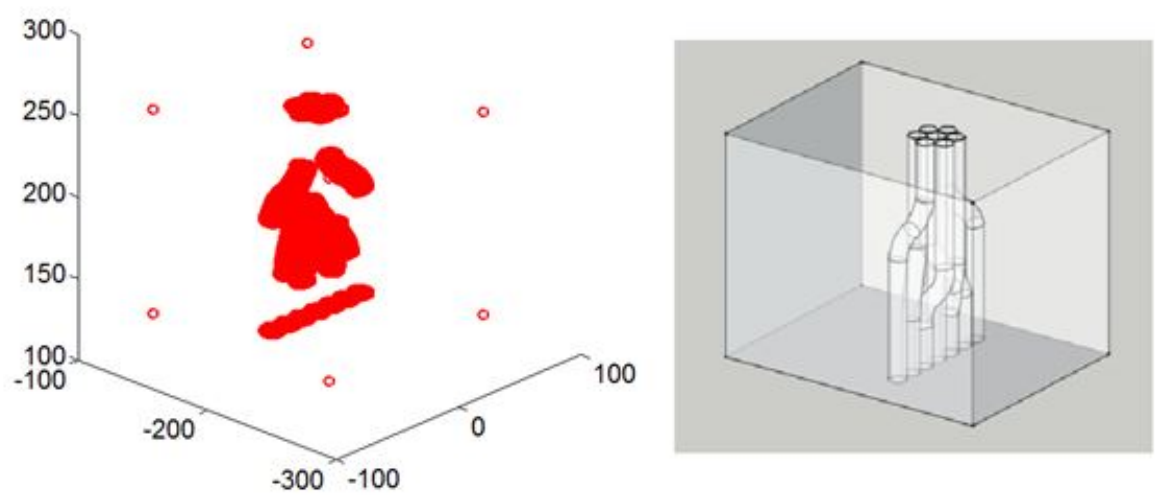

Figure 5.5: Side-by-side of the model (right) and the extracted triangle verticies (left). 
A generic model of a linear array of 2 waveguides being coupled into a single aperture was created using Google SketchUp, which allows exporting of .stl file types. The .stl file was read into Matlab using the algorithm for generating machine movements. Plotting the machine movements produces the graph shown in Figure 5.6.

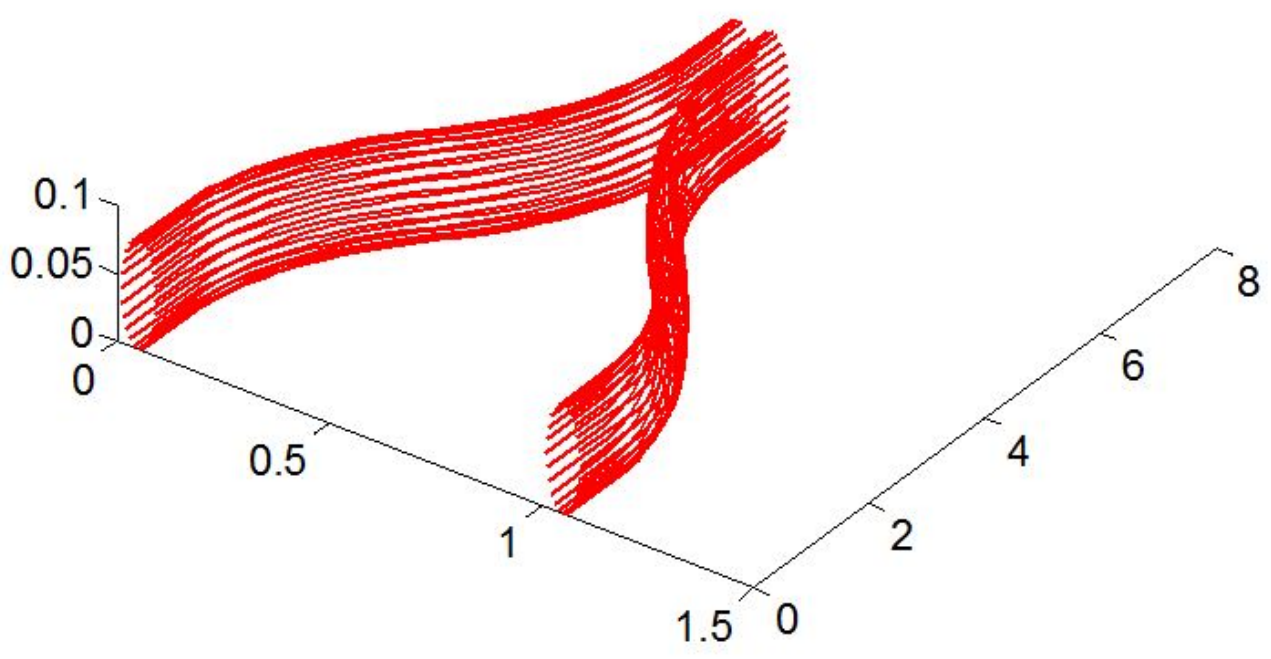

Figure 5.6: Modeled machine movements to create a 2 into 1 aperture waveguide structure.

From the generate machine movements shown in Figure 5.6, a waveguide structure was written in $\mathrm{ZnSe}$ using the sorted machine movements. The resultant structure can be seen in Figure 5.7 and Figure 5.8. 


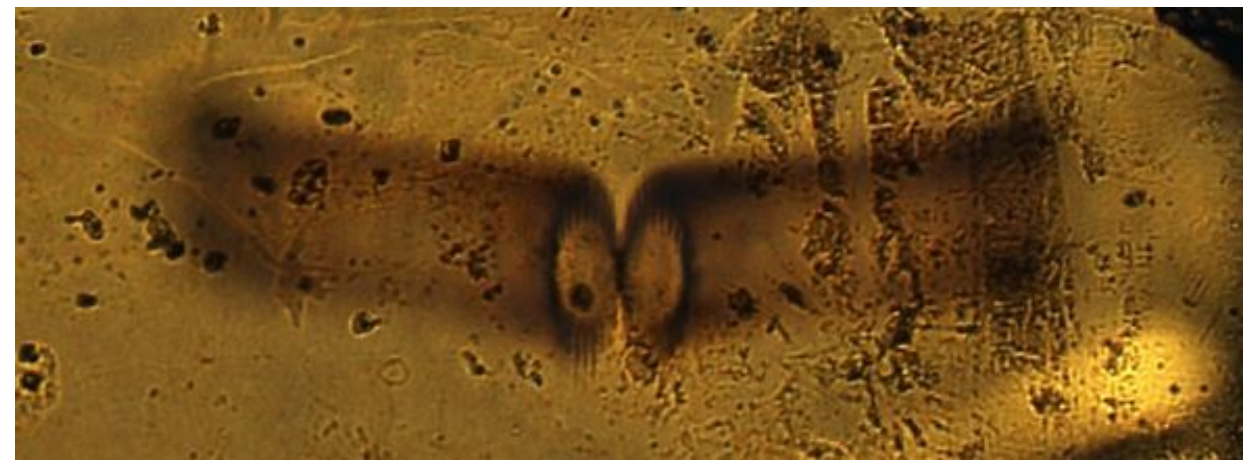

Figure 5.7: Waveguide coupler from the machine movements in Figure 5.6. The picture shows the waveguide sample focused near the $7 \mathrm{~mm}$ depth.

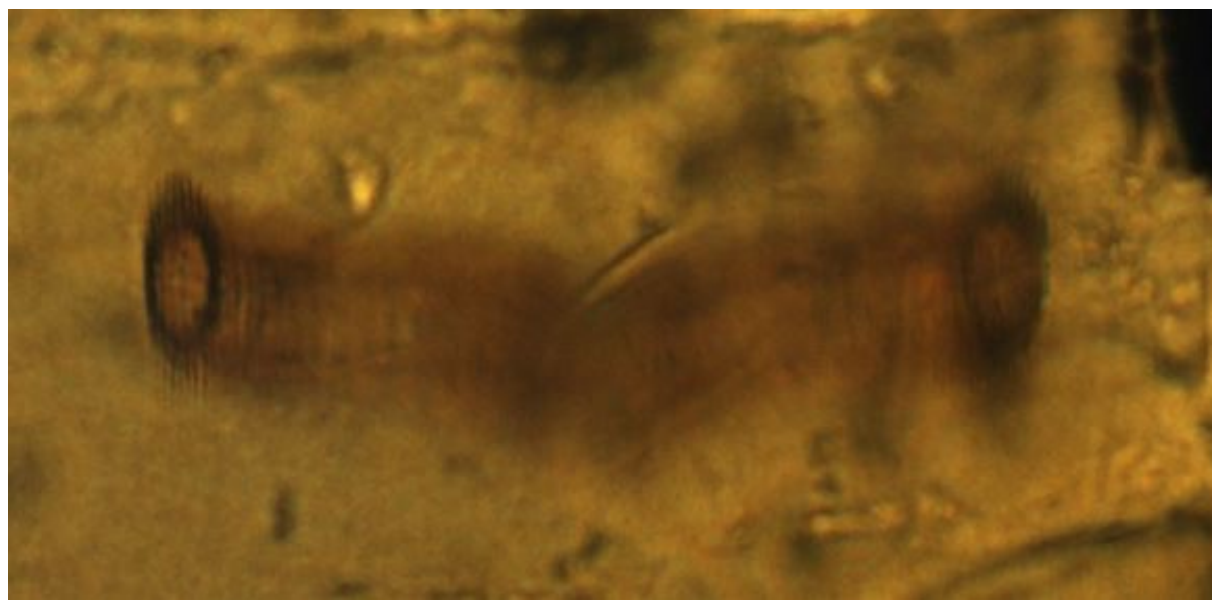

Figure 5.8: Waveguide coupler from the machine movements in Figure 5.6. The picture shows the waveguide sample focused near the $0 \mathrm{~mm}$ depth.

The main issue with this structure is that the losses are higher than expected. The measured propagation loss of a single curved waveguide at $1908 \mathrm{~nm}$ is in excess of $3 \mathrm{~dB} / \mathrm{cm}$, and the observed mode exiting the waveguide has extremely poor quality, Figure 5.9. The cause of the high loss in these structures is likely due to the bend losses of these waveguides. 


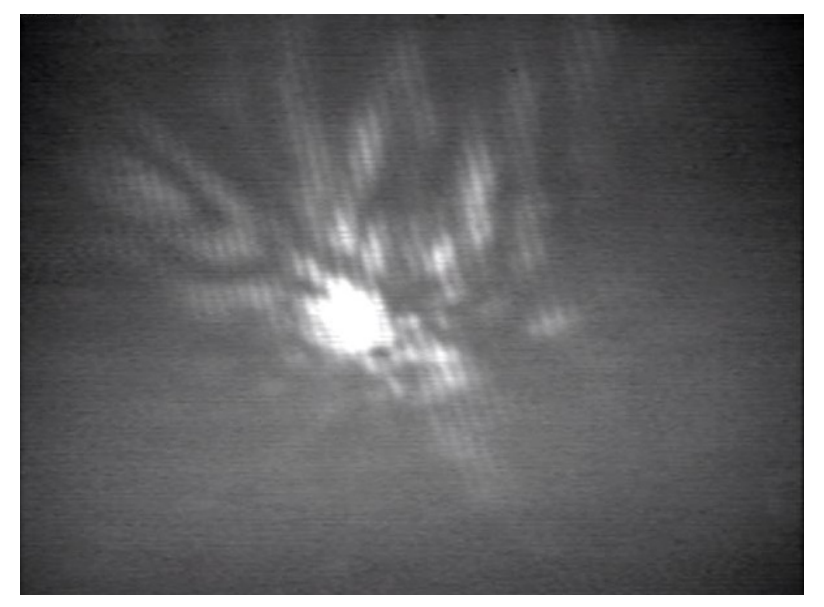

Figure 5.9: Propagating mode observed after propagating in the waveguides shown in Figure 5.7.

In order to test this theory, several waveguides were inscribed in $\mathrm{ZnSe}$ with varying bend radii from $50 \mathrm{~mm}$ to $500 \mathrm{~mm}$. However, a peculiar issue arose from the ULI waveguides. After approximately half of the translation distance, the laser would stop modifying the material Figure 5.10. The lack of modification would consistently happen regardless of the parameters used for inscription.

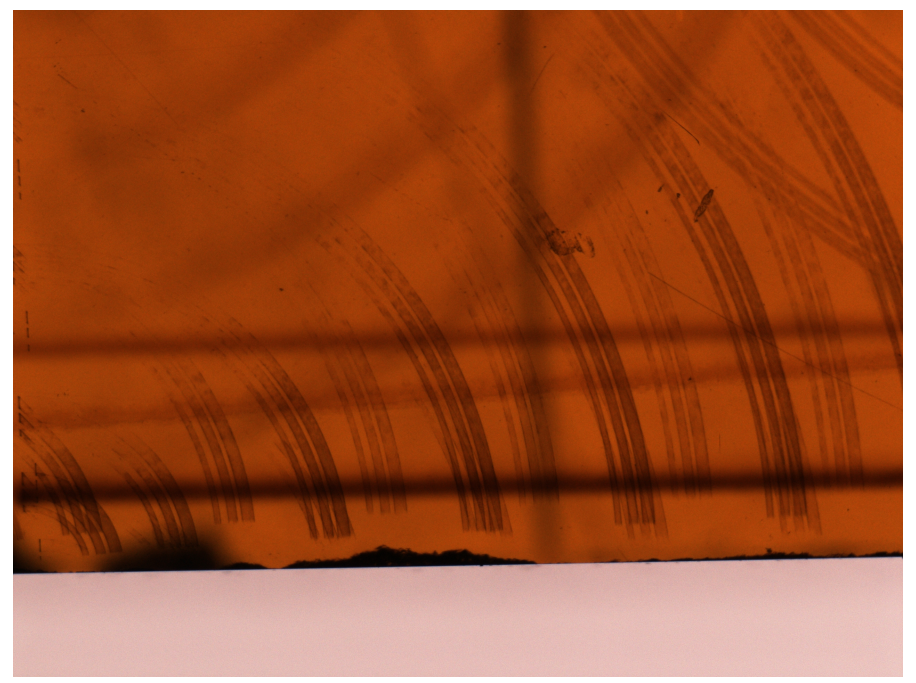

Figure 5.10: Waveguides inscribed in ZnSe with varying radii of curvature from $50 \mathrm{~mm}$ to $500 \mathrm{~mm}$. The inscription process fails to modify the $\mathrm{ZnSe}$ approximately halfway through the translation distance. The picture shows several attempts at creating curved waveguides. 
By adjusting the polarization direction of the inscription laser such that the polarization direction was kept perpendicular to the propagation direction of the waveguide, the modification was maintained over the entire translation direction, Figure 5.12. Several authors have shown a dependence on the inscription laser polarization on the type of modification obtained during ULI[56, 57]. Depending on the laser polarization, nanogratings can be formed inside of a material, where the grating direction is perpendicular to the electric field polarization direction, Figure 5.11. This same type of interaction could explain the need for polarization rotation during inscription of curved waveguides in $\mathrm{ZnSe}$. If nanogratings exist in the ULI structures created in $\mathrm{ZnSe}$, then there could be a difference
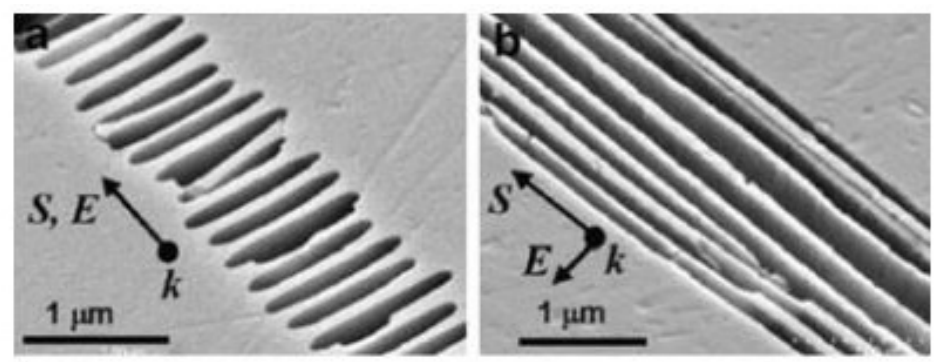

Figure 5.11: Scanned electron microscope image of nanogratings formed at $65 \mu \mathrm{m}$ depth (sample cleaved and polished at writing depth) with polarization parallel (a) and perpendicular (b)to the scan direction. This picture has been reproduced from Bhardwaj et al. [57]

in the inscription parameters needed to induce a permanent index change inside of the material for perpendicular polarization directions. However, the propagation losses of these waveguides were still larger than expected. The losses, measured with the techniques outlined in Chapter III, varied from $2.8 \mathrm{~dB} / \mathrm{cm}$ for a radius of curvature of $5 \mathrm{~mm}$ to $1.9 \mathrm{~dB} / \mathrm{cm}$ for a radius of curvature of $500 \mathrm{~mm}$. The losses were measured at a wavelength of $1.9 \mu \mathrm{m}$.

Despite the high losses, several 50/50 waveguide beam splitters were fabricated utilizing the algorithm shown in Appendix C. The resulting couplers can be seen in Figure 5.13. 


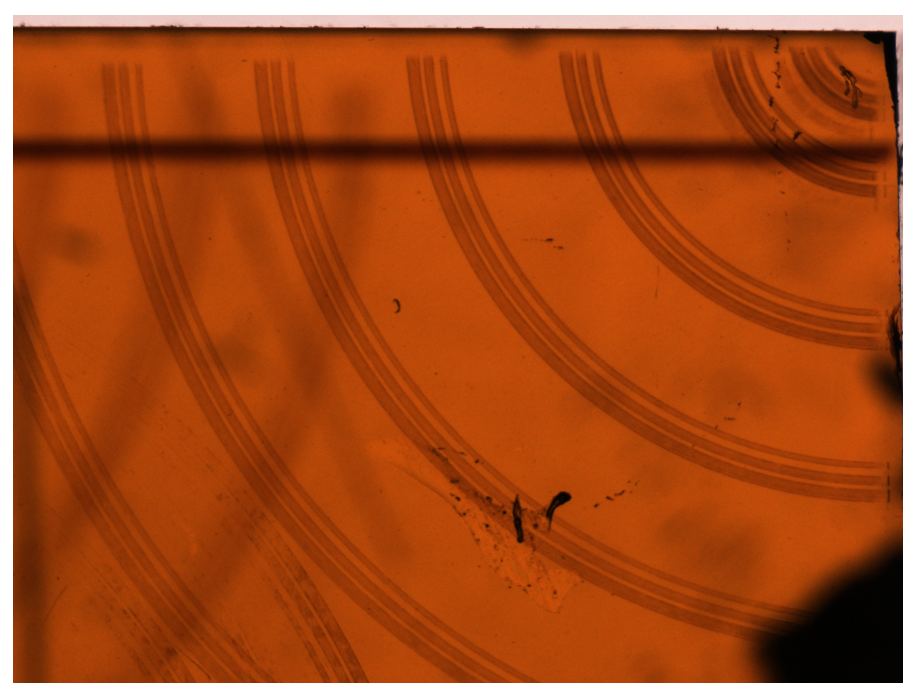

Figure 5.12: Waveguides inscribed in ZnSe with varying radii of curvature from $50 \mathrm{~mm}$ to $500 \mathrm{~mm}$. The polarization direction of the inscription beam was rotated to keep the polarization direction perpendicular to the propagation direction of the waveguide.

With this proof of concept, more exotic configurations can now be investigated. Such a configuration can be seen in Figure 5.14. This figure shows a preliminary design to couple light from an array of 9 QCLs into a single aperture. One of the issues with QCLs is that high power operation requires several devices to be combined in a linear array. Individual QCLs have large, asymmetric beam divergence. Fast axis beam divergence is in the range of 40-60 degrees, which limits the usefulness of the device in the far field.

Utilizing the model shown inFigure 5.14, an array of waveguides can be produced, which couple to a single aperture, Figure 5.15. However, since the inscription depth changes by several hundred microns, the quality of the modification changes with depth. The lower modification, near the bottom of Figure 5.15, shows distinct signs of non-critical modification, which produces spurious weak modification. There are two ways that this can be corrected. First, the structure can be moved closer to the surface of the sample, which decreases the pulse dispersion and decreases the 


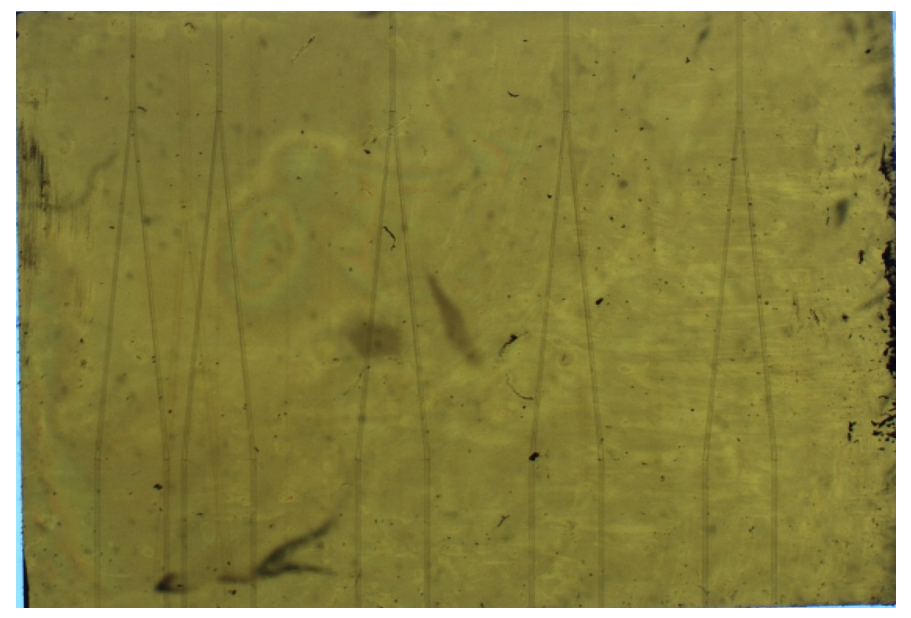

Figure 5.13: 50/50 waveguide splitters inscribed in a $10 \mathrm{~mm} \times 7 \mathrm{~mm}$ sample of ZnSe.

aberrations on the ULI beam. Second, in inscription power can be preferentially increased for the lower waveguides. Both of these techniques will be investigated future research.

\subsection{Conclusions}

The ability to create arbitrarily shaped geometries utilizing ULI relies heavily on the ability of structures to be translated into physical machine movements. These structures can be extremely difficult to hard-code into a form that the translation stages can interpret. An algorithm was developed in MATLAB to generate machine movements for arbitrarily complex structures by analyzing the .stl file created from the CAD drawing. The waveguides that were inscribed with the output of the algorithm matched the CAD drawings of the structures. However, the inscribed waveguides exhibited higher losses than expected, with the smallest radii of curvature waveguides having losses of $\approx 2.8 \mathrm{~dB} / \mathrm{cm}$ at $1.9 \mu \mathrm{m}$. The high losses of these structures could be caused by increased overlap of the evanescent field with the ULI modifcation region. 


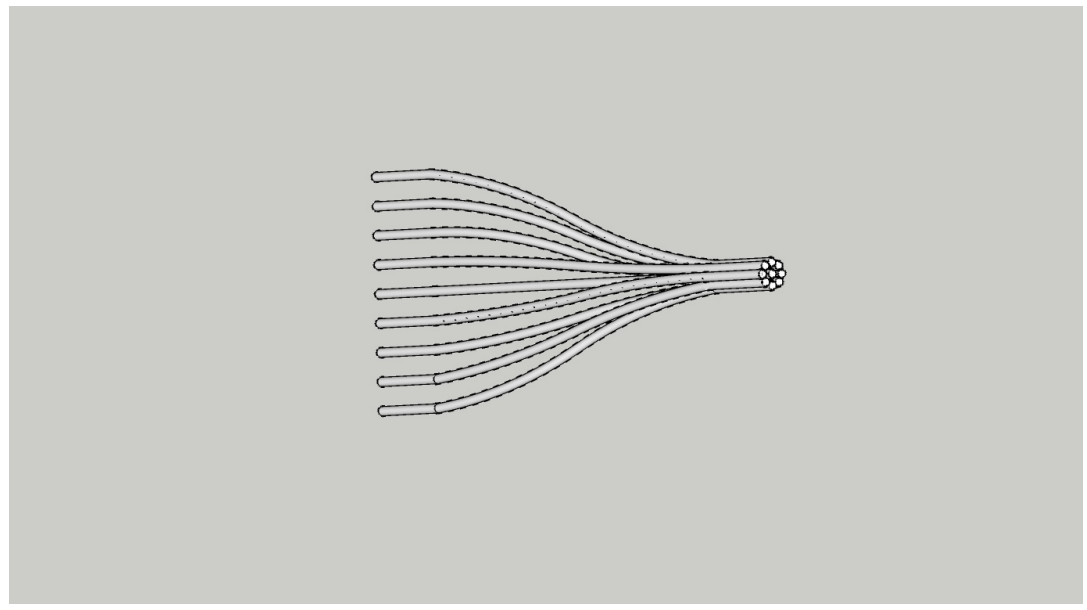

Figure 5.14: Model of the 9 to 1 coupler created to scale in SketchUp.

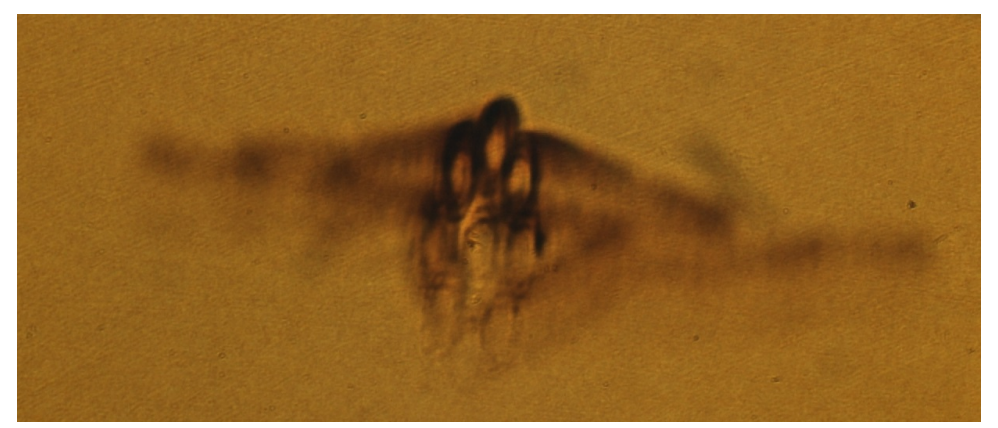

Figure 5.15: Inscribed strcuture in $\mathrm{ZnSe}$ using the 9 to 1 coupler model from SketchUp. The waveguides show spurious modification at greater depths. 


\section{CHAPTER VI}

\section{TRANSITION METAL ULI LASERS}

\subsection{Power Scaling}

Compact, Mid-IR lasers operating in the $2-5 \mu \mathrm{m}$ are a vital, enabling technology for a variety of applications including remote sensing [58], medical procedures [59], and spectroscopy [60]. These applications provide motivation for the advancement and optimization of mid-IR sources. The eventual aim of these devices are for efficient laser sources, which can be easily incorporated into existing systems and technologies. Transition metal (TM) ions doped into zinc based chalcogenides, notably zinc selenide $(\mathrm{ZnSe})$ and zinc sulfide $(\mathrm{ZnS})$, have many traits, which make them desirable candidates for many applications. The relitavely large absorption and emission cross-sections broadened by crystal field splitting of the allowed electronic levels of the TM ions produce an adaptable medium capable of absorbing and emitting laser radiation over a large spectral range[4]. Specifically, chromium and iron dopants have been shown to readily emit over the $2-3 \mu m[6,5]$ and $4-5 \mu m[61,62]$ regions respectively. In addition, these sources have been scaled to multi-Watt level output power in non-guided wave devices. A summary of published power level records can be seen in Table 6.1 [29].

Many of the intended applications of these sources are fielded in non-laboratory settings which are sensitive to vibration, temperature changes, humidity, and particulates in the air. In order to improve the reliability and robustness of these systems, minimization of free-space optics are required 
Table 6.1: Summary of bulk laser power records

\begin{tabular}{ccc}
\hline Author & Material & Power \\
\hline Martyshkin[10]* & Fe:ZnSe & $35 \mathrm{~W}$ \\
Evans[63] & Fe:ZnSe & $0.84 \mathrm{~W}$ \\
Mirov[9]* & Cr:ZnSe & $57 \mathrm{~W}$ \\
Moskalev[64] & Cr:ZnSe & $135 \mathrm{~W}$ \\
\hline
\end{tabular}

* denotes pulsed operation

in order to decrease sensitivity to the environement. Thus, confining operation to a guided-wave configuration is desirable. Guided-wave operation is usually realized in a fiber form, but due to the low sublimation point of $\mathrm{ZnSe}$ and $\mathrm{ZnS}\left(900^{\circ} \mathrm{C}\right.$ [65] and $1200^{\circ} \mathrm{C}$ [66] respectively) and due to the absence of a glass-phase with crystalline materials, pulling of the material into a fiber is impossible with the current technology [67]. However, other technologies exist to create ZnSe fibers with losses less than $1 \mathrm{~dB} / \mathrm{cm}$ [68], relying on chemical vapor deposition (CVD) of crystalline material. However, these fibers have been limited to small lengths and do not support a Gaussian mode due to the CVD geometry. An alternative method to create guided-wave structures was demonstrated by Okhrimchuk et al. [27], which uses femtosecond laser interactions to modify the index of bulk material to create waveguide structures. This technology has been demonstrated in both Fe:ZnSe [31] and Cr:ZnSe [69] with limited output power. The current maximum average powers obtained from these sources can be seen in Table 6.2. Power scaling of both Cr:ZnSe and Fe:ZnSe waveguides to power levels greater than $2 W$ is desired. In order to do this, an overall decrease to the propagation losses of the devices needs to be achieved, in addition to careful thermal management in order to allow higher pumping rates in the materials. 
Table 6.2: Summary of waveguide laser power records

\begin{tabular}{ccc}
\hline Author & Material & Power \\
\hline Berry[30] & Cr:ZnSe & $1.7 \mathrm{~W}$ \\
Lancaster[31] & Fe:ZnSe & $0.07 \mathrm{~W}$ \\
McDaniel[70]* & Cr:ZnSe & $0.18 \mathrm{~W}$ \\
\hline
\end{tabular}

$*$ denotes pulsed operation

\subsubsection{Fabrication}

Fabrication of ULI waveguide structure relies on precise control of both an automated 3-axis translation stage and the laser parameters of an mode-locked laser source. Waveguides were inscribed in bulk Fe:ZnSe and Cr:ZnSe using a chirped pulse, amplified Yb-fiber laser (IMRA $\mu$ Jewel D1000) operating at $1047 \mathrm{~nm}$. The waveguides were inscribed at $100 \mathrm{kHz}$ repetition rate with a pulse width of $850 \mathrm{fs}$ and 1-5 overwrites. The beam was focused into the sample using a 0.68 NA lens with a focal length of $4.1 \mathrm{~mm}$. The sample was translated using an XYZ translation stage (Aerotech Model:A3200) moving with a velocity of $10 \mathrm{~mm} / \mathrm{s}$. The resultant cladding structure for the best performing waveguides can be seen in Figure 6.1. The Cr: $\mathrm{ZnSe}$ structure was designed to have a core diameter of $120 \mu \mathrm{m}$ composed of 100 individual elements. Several sets of waveguides were inscribed in the bulk sample of Cr:ZnSe for testing. The inscription parameters were varied for each waveguide set to provide a wide variety of waveguides for testing. The actual waveguide diameter of the Cr:ZnSe was smaller than the designed diameter due to elongation of the individual waveguide elements. A similar structure was created inside of a bulk Fe:ZnSe sample except with a diameter of $80 \mu m$. 


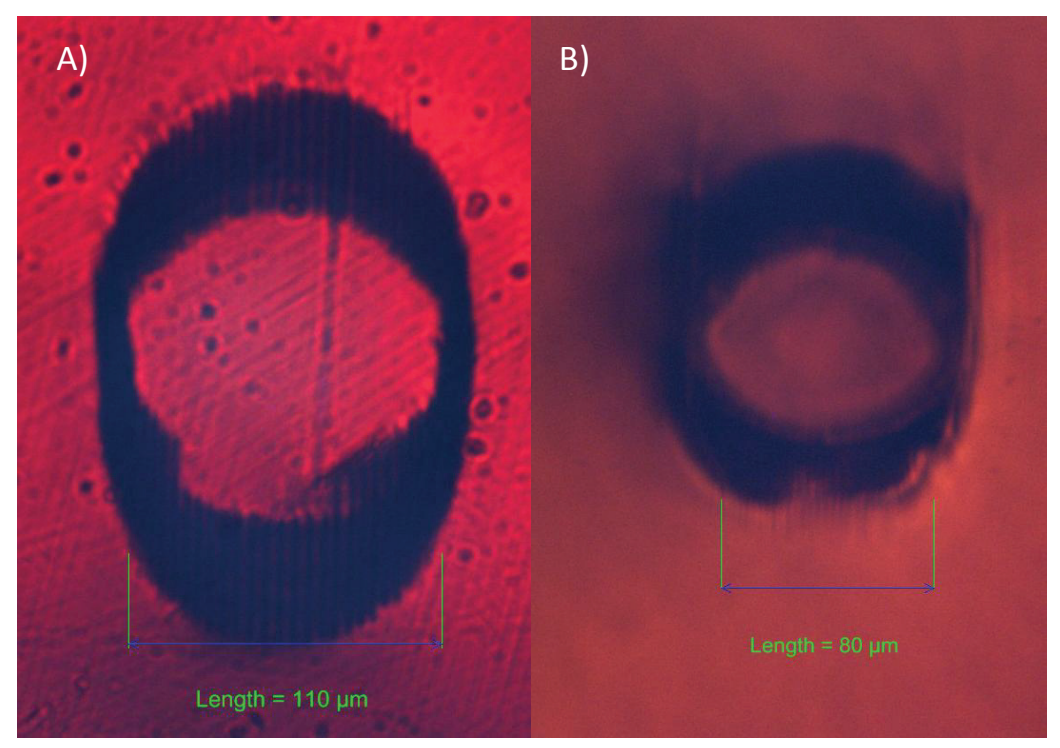

Figure 6.1: Cladding structure created in $\mathrm{Cr}: \mathrm{ZnSe}(\mathrm{A})$ and $\mathrm{Fe}: \mathrm{ZnSe}(\mathrm{B})$ using the ULI setup described in the previous section. The waveguides had a diameter of approximately $110 \mu \mathrm{m}$ (A) and $80 \mu \mathrm{m}$ (B) respectively. Photo was taken looking into the direction of propagation.

The material specific properties for Fe:ZnSe and Cr:ZnSe change the required pulse energy for modification significantly depending on the dopant type and concentration. Small changes in the linear absorption of the sample can decrease the fluence at the focus of the beam. Decreased fluence compounded with the nonlinear self focusing caused by the large $\chi^{(3)}$ coefficient $\left(1 \times 10^{-12}\right.$ esu [71]), can result in poor quality waveguides if the inscription parameters are not precisely controlled. Figure 6.2 shows an example of poor modification in $\mathrm{Cr}: \mathrm{ZnSe}$, obtained by non-optimal inscription parameters. Comparing Figure 6.1 and Figure 6.2, the modification under non-optimal parameters causes the modification regions to blur together and encroach on the guiding region, increasing propagation losses of the waveguide. 


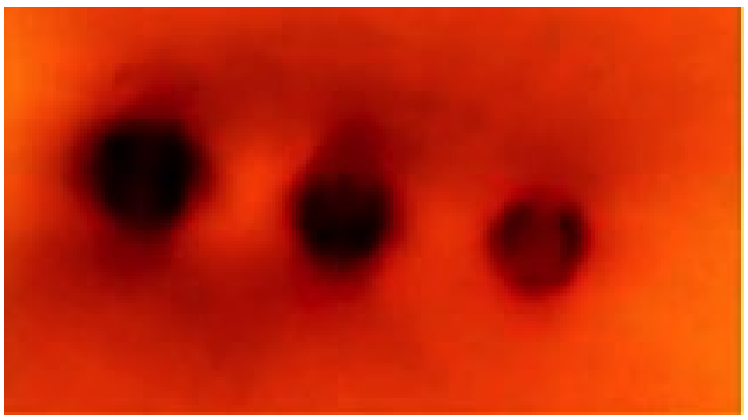

Figure 6.2: Waveguides inscribed in Cr:ZnSe under non-optimal inscription parameters. The waveguides range in size from $40 \mu \mathrm{m}$ to $120 \mu \mathrm{m}$

The pulse energy required to obtain optimal modification in Cr:ZnSe was $2.4 \mu J$ and $0.8 \mu J$ for Fe:ZnSe. The absorption cross-sections of Cr:ZnSe and Fe:ZnSe can be seen in Figure 6.3, where the green circles represent the absorption cross-section at the inscription laser wavelength.

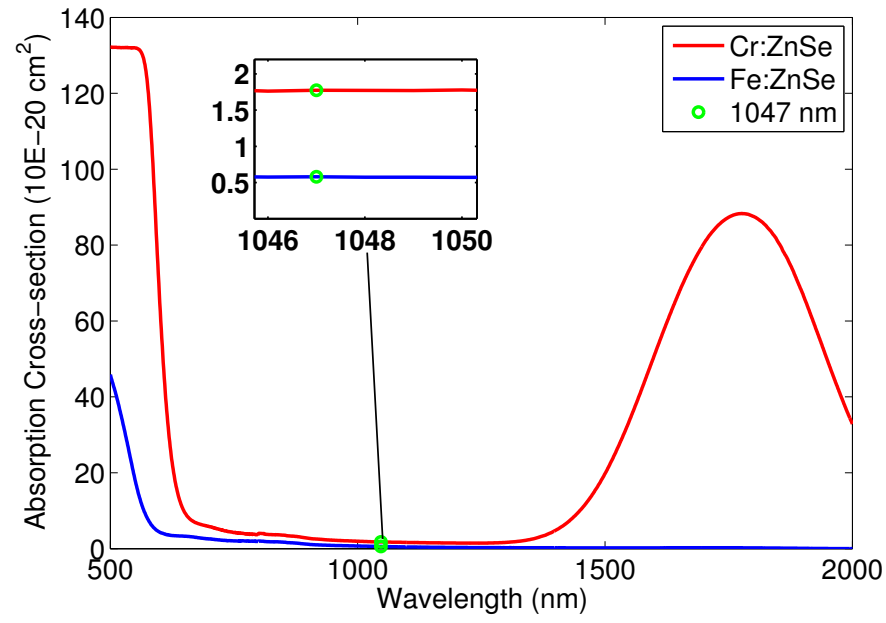

Figure 6.3: Measured absorption cross-sections of Cr:ZnSe and Fe:ZnSe as a function of wavelength. The cross-section was calculated with the quoted sample concentration. 
The samples, obtained from IPG Photonics, were doped with $8.2 \times 10^{18} \mathrm{~cm}^{-3}$ of chromium and $1.4 \times 10^{19} \mathrm{~cm}^{-3}$ of iron ions respectively. The samples lengths were $9 \mathrm{~mm}$ for the $\mathrm{Cr}: \mathrm{ZnSe}$ sample and $10.1 \mathrm{~mm}$ for the Fe:ZnSe sample. The inscription laser's wavelength is denoted by the circle on the graph. Upon closer examination of the transmission spectra, it can be seen that Cr:ZnSe has a much higher absorption cross-section at $1047 \mathrm{~nm}$ than Fe:ZnSe. The difference in absorption can explain the difference in the pulse energy required to produce modification due to the linear absorption loss of the inscription beam propagating through the sample. Additionally, the loss in pulse energy from the linear absorption also affects the nonlinear interactions induced in the sample. It should be noted that the required pulse characteristics for inscription in Fe:ZnSe follow the parameters required for inscription of undoped $\mathrm{ZnSe}$ more closely than the parameters required for inscription in Cr:ZnSe. The similarity of inscription parameters for undoped material and Fe:ZnSe indicates that the presence of chromium in the sample is the cause of the difference in the inscription parameters between Fe:ZnSe and Cr:ZnSe.

\subsubsection{Experimental Setup}

Optimal operation parameters for Cr:ZnSe and Fe:ZnSe have been investigated by many authors $[72,73,74,75]$. These results were taken into consideration to obtain optimal operation of the waveguide lasers. Unfortunately, Fe:ZnSe only operates efficiently in a $\mathrm{CW}$ configuration at cryogenic temperatures due to phonon assisted quenching of the radiative lifetime [76]. In early demonstrations of TM waveguide lasers, laser mirror coatings were kept on external optics to allow versatility for testing $[31,30]$ and evaluation of perforamce by techniques such as Findlay-Clay [77] and Rigrod analysis[78]. However, building upon previous research, we can determine the optimal operating parameters for each material (i.e. optimal outcoupler reflectivity, operating temperature, tolerable losses etc.) $[5,31]$. Knowledge of the ideal operating parameters allows for minimization of free-space optics to prevent unnecessary losses from external optics. Laser cavity mirrors were 
directly coated onto the end facets of the samples, thus making the sample the entire optical cavity. Figure 6.4 shows the cavity configurations for $\mathrm{Cr}: \mathrm{ZnSe}$ and Fe:ZnSe waveguide power scaling efforts. For Fe:ZnSe, L1 and L2 were $4 \mathrm{~cm}$ focal length broadband $(2-5 \mu \mathrm{m})$ anti-reflective (AR) lenses, W1 and W2 were broadband $(2-5 \mu m)$ AR coated windows, and the solid and dotted lines represent the dichroic incoupler and outcoupler coatings on the end face of the crystal. The incoupler coating was designed to be AR at $2.94 \mu \mathrm{m}$ and HR at $4-4.2 \mu m$. The outcoupler coating was designed to be $40 \%$ reflective at $4-4.2 \mu \mathrm{m}$. The Fe:ZnSe crystal was wrapped in indium and placed in a vacuum dewar cooled by liquid nitrogen to $77 \mathrm{~K}$. Similarly, the Cr:ZnSe setup consisted of dichroic incoupler and outcoupler coatings in addition to the broadband AR $10 \mathrm{~cm}$ focal length lenses L1 and L2. The incoupler coating was designed to be AR at $1.9-2.1 \mu \mathrm{m}$ and HR at $2.3-3 \mu m$. The outcoupler was designed to be AR at $1.9-2.1 \mu m$ and $70 \%$ reflective at $2.3-3 \mu m$. The Cr:ZnSe sample was wrapped in indium to improve thermal contact with the heatsink. The heatsink was chilled to $11^{\circ} \mathrm{C}$, which was slightly above the point where water vapor started to condense on the sample. The pump sources used for pumping of Cr:ZnSe and Fe:ZnSe were a thulium fiber laser operating at $1.9 \mu \mathrm{m}$ and a erbium fiber laser operating at $2.9 \mu \mathrm{m}$. The samples were mounted on a 5-axis translation stage capable of pitch and yaw control in addition to XYZ translation. The 5-axis stage allowed for fine adjustment of the input pump coupling into the waveguide to maxamize the laser output. Nominally, the pump laser was focused to a $1 / e^{2}$ diameter of $80 \mu m$ for the Cr:ZnSe waveguide and $50 \mu m$ for the Fe:ZnSe waveguide. The focal spot sizes were kept smaller than the waveguide diameter to prevent interaction of the pump beam with the cladding structure, which could cause increased laser losses due to absorption or scattering from the cladding. Small adjustments were made to the distance between the pump focusing lens and the sample to maximize the output power from the laser. 

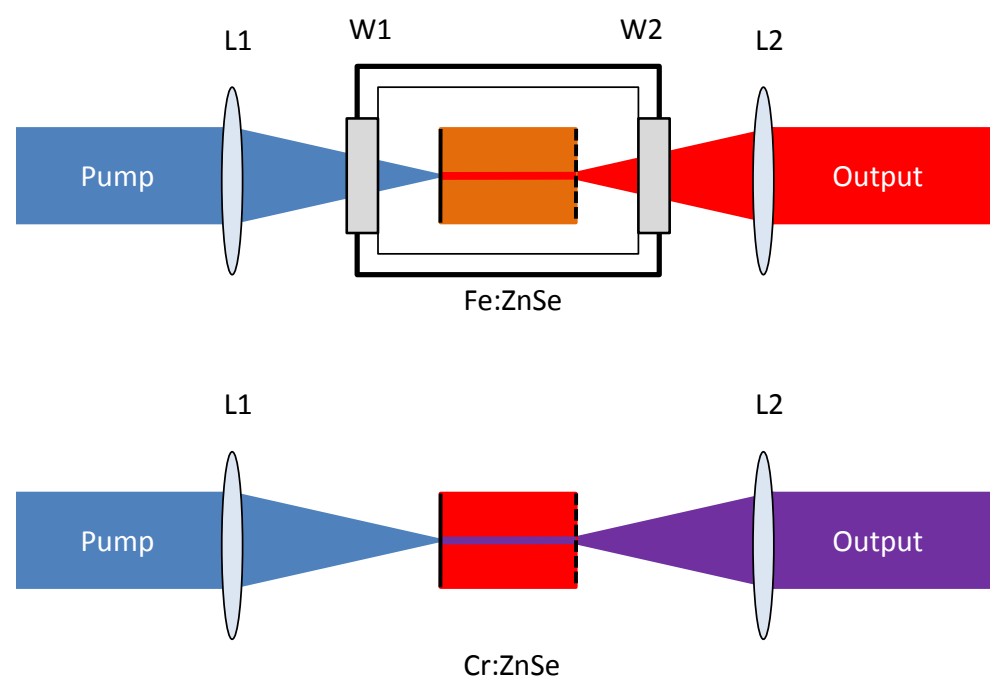

Figure 6.4: Cavity configurations for Fe:ZnSe and Cr:ZnSe waveguide lasers.

\subsubsection{Special Considerations}

For high power $\mathrm{CW}$ operation, several factors must be taken into consideration when applying focused, laser power to the end facet of a crystal. First, the intensity must be kept below the damage threshold of the coating material. However, coating manufacturers rarely have $\mathrm{CW}$ damage threshold values for their optical coatings. The main cause of failure for optical coatings under $\mathrm{CW}$ irradiation is typically thermal proceeses [79], thus thermal management additionally helps prevent coating damage. Following Equation 6.1, the intensity of a given spot size increases linearly as a function of power for a given spot size, where $P$ is the incident power, $I$ is the intensity, and $r$ is the radius of the pump spot. With a conservative estimate of $200 \mathrm{~kW} / \mathrm{cm}^{2}$ damage threshold for CW operation of the optical coatings, pump laser power has to be kept under $\approx 2 W$ for a spot size of $80 \mu m(\mathrm{Cr}: \mathrm{ZnSe})$ and $\approx 10 W$ for a spot size of $50 \mu m(\mathrm{Fe}: \mathrm{ZnSe})$. In practice, these values will be less due to localized heating and differential thermal expansion of the thin film coating and the bulk 
laser sample.

$$
I=\frac{P}{\pi r^{2}}
$$

Additionally, we must consider the effect of the waveguide diameter on the threshold for laser operation. Equation 6.2 shows the threshold pump power for a simple four-level laser [80], where $\mathrm{h}$ is Plank's constant, $\nu$ is the frequency of the pump laser, $A$ is the area of the pump spot, $l$ is the passive losses for the resonator, $\eta$ is the conversion efficiency, $\tau$ is the upper state lifetime, and $\sigma$ is emission cross-section of the material. The graph shown in Figure 6.5 shows an approximation of the pump power required to obtain lasing from samples of $\mathrm{Cr}$ and $\mathrm{Fe}: \mathrm{ZnSe}$. Published values for the cross-section and lifetime were used $[4,81]$, Table 6.3. In addition, a equivalent loss of approximately $1 \mathrm{~dB} / \mathrm{cm}$ was measured for both waveguide samples at a wavelength of $2.4 \mu \mathrm{m}$. Threshold pump values of $350 \mathrm{~mW}$ and $50 \mathrm{~mW}$ were estimated for Cr:ZnSe and Fe:ZnSe respectively.

$$
P_{t h}=\frac{h \nu A l}{\eta \tau \sigma}
$$

Table 6.3: Parameters used for calculation of the threshold pump power.

\begin{tabular}{|c|c|c|}
\hline & Cr:ZnSe & Fe:ZnSe \\
\hline$\sigma_{e m}$ & $90 \times 10^{-24} m^{2}$ & $70 \times 10^{-24} m^{2}$ \\
\hline$\tau$ & $6 \mu s$ & $60 \mu s$ \\
\hline$\nu$ & $1.5 \times 10^{14} H z$ & $1.2 \times 10^{14} H z$ \\
\hline$\eta$ & $77 \%$ & $71 \%$ \\
\hline$l$ & $20 \%$ & $20 \%$ \\
\hline
\end{tabular}




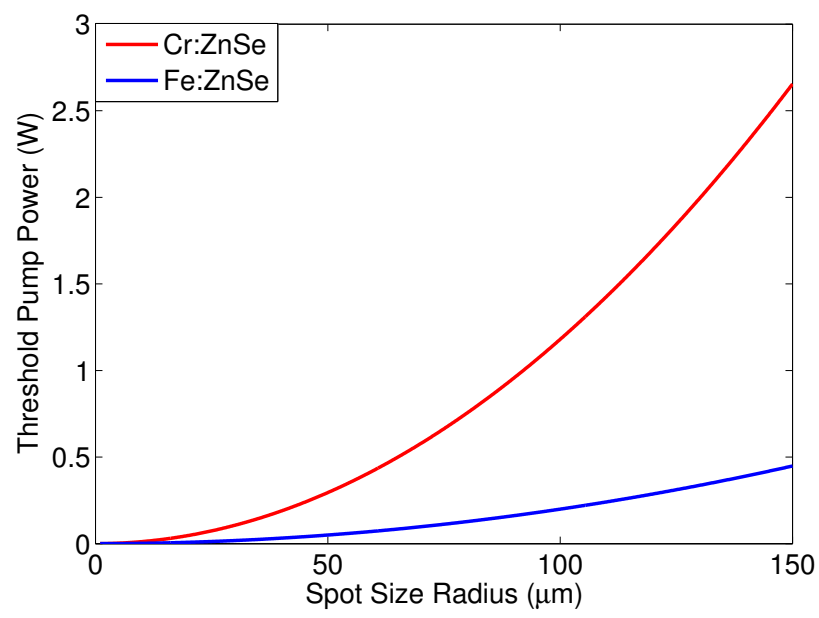

Figure 6.5: Calculation of the threshold pump power required for inversion in a material as a function of spot size.

\subsubsection{Cr:ZnSe Results}

Utilizing the setup shown in Figure 6.4, the Cr:ZnSe sample was pumped with a thulium fiber laser (IPG Photonics TLR-40-1910-LP). An Electrophysics PV-320 camera was used to align and maximize the pump light coupled into the waveguide structure. The sample was mounted on a 5axis translation stage to align and maximize the light coupled into the waveguide structure. Using a dichroic splitting mirror, AR at $1.9 \mu \mathrm{m}$ and HR at $2.3-3 \mu \mathrm{m}$ and for an angle of incidence of $45^{\circ}$, the fluorescence signal from the waveguide was maximized utilizing an extended range InGaAs detector. Lasing occurred around $400 \mathrm{~mW}$ pump for Cr:ZnSe. The sample was translated perpendicular to the pump beam direction. Light was coupled into adjacent waveguides to identify the best performing waveguide (i.e. the waveguide that produces the highest output power for a set input power). The adjacent waveguides had a variety of different inscription parameters including number of overscans, scan speed, pulse energy, and core size. Figure 6.7 shows the output of the best performing waveguide. A maximum output of $5.2 \mathrm{~W}$ was obtained from $11.95 \mathrm{~W}$ of input power, 
with a slope efficiency of $41 \%$. While not the highest reported slope efficiency from a Cr:ZnSe device [5], a slope efficiency of $41 \%$ is commonly achieved in literature [82, 73]. Additionally, Figure 6.7 shows no thermal rollover indicating that these waveguide structures have not reached the thermal limit of performance. It should be noted that the input power was referenced to the power incident on the front face of the crystal, not the absorbed power as commonly found in literature. A higher slope efficiency could be obtained if the absorbed power was used for the slope efficiency. Unfortunately, at $12.2 \mathrm{~W}$ of input power the sample coating was damaged, which rendered the waveguide inoperable, Figure 6.6.

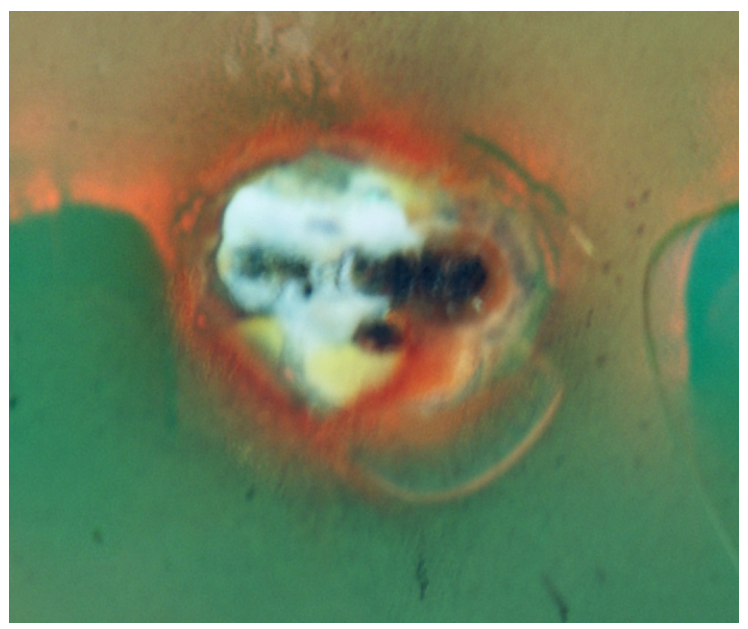

Figure 6.6: Facet damage that occurred during CW pumping of the Cr:ZnSe waveguide.

The Cr:ZnSe results obtained from this work can be compared to the work previously investigated by Berry et al.[30], which identified thermal quenching as the limiting factor in power scaling of Cr:ZnSe waveguide lasers. Several factors affect the internal temperature of the waveguide. The external cooling of the sample lowers the internal operating temperature of the waveguide allowing higher operating powers to be reached before thermal quenching occurs. Previously, the sample was cooled to $15^{\circ} \mathrm{C}$, while in this work the sample was cooled to $11^{\circ} \mathrm{C}$. The waveguide for this effort 
was also closer to the cooling facet. The waveguide was inscribed at a depth of $100 \mu m$ while the waveguide previously investigated by Berry et al. was inscribed at a depth of $200 \mu \mathrm{m}$. In addition, the extraction efficiency of the demonstrated work was increased $18 \%$ compared to the previous work. The increased rate of energy extraction from the gain medium also decreases the heat load on the waveguide.

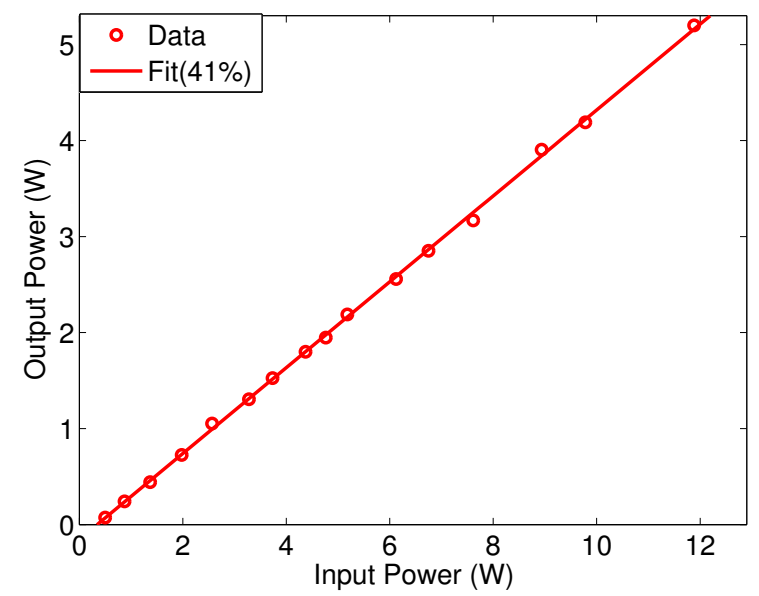

Figure 6.7: Optical performance of the $111 \mu m, \mathrm{Cr}: \mathrm{ZnSe}$ waveguide laser.

The output mode of the Cr:ZnSe waveguide can be seen in Figure 6.8(A), which was a scaled and resized image of the mode. From Figure 6.8(A), we can see that the output mode of the Cr:ZnSe waveguide laser was highly multimode, with some slight asymmetry. Translating the beam vertically out of the waveguide, Figure 6.8 (B), shows the unscaled, unguided spot from the pump laser transmitted through the same optics. The unguided beam shown in Figure 6.8(B) produced no laser output from the system due to the stability condition of the laser cavity. From direct measurement of output beam divergence, we can estimate the numerical aperature (NA) of the waveguide to be 0.37. For a symmetric, cylindrical waveguide the number of guided modes can be estimate by the 
normalized frequency of the waveguide

$$
\begin{gathered}
V=\frac{2 \pi a}{\lambda} \sqrt{n_{\text {core }}^{2}-n_{\text {clad }}^{2}}, \\
N=\frac{4 * V^{2}}{\pi^{2}} .
\end{gathered}
$$

In Equation 6.3, $V$ is the normalized frequency, $a$ is the radius of the core, $\lambda$ is the wavelength of the guided light, $\mathrm{N}$ is the number of guided modes, and $n_{\text {core }}$ and $n_{\text {clad }}$ are the indices of refraction for the core and cladding respectively. It should be noted that $\sqrt{n_{\text {core }}^{2}-n_{\text {clad }}^{2}}$ is equal to the NA of the waveguide. The number of guided modes of the waveguide, using 0.37 for the NA, was $\approx 1000$. In practice, this number is less due to the increased interaction with the cladding region for higher order modes and due to the asymmetry of the waveguide profile. The output spectrum of the Cr:ZnSe waveguide laser can be seen in Figure 6.9. The output wavelength was centered at $2522 \mathrm{~nm}$ with a spectral bandwidth of $20 \mathrm{~nm}$. The output of the waveguide laser is red-shifted due to typical bulk Cr:ZnSe. The cause of the red-shift is due to the decrease propagation losses at high wavelengths. Compared to typical bulk lasers with no wavelength selective optics (i.e. etalons, tuning elements, filters), the spectral bandwidth is decreased by approximately $30 \mathrm{~nm}$.

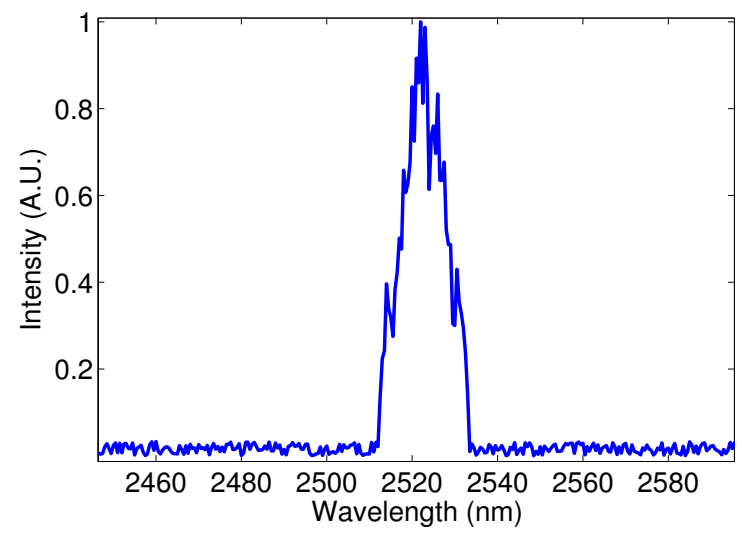

Figure 6.9: Output spectrum of the Cr:ZnSe waveguide laser centered at $2522 \mathrm{~nm}$ measured with an Acton SP2500 spectrometer with a $0.5 \mathrm{~nm}$ resolution. 


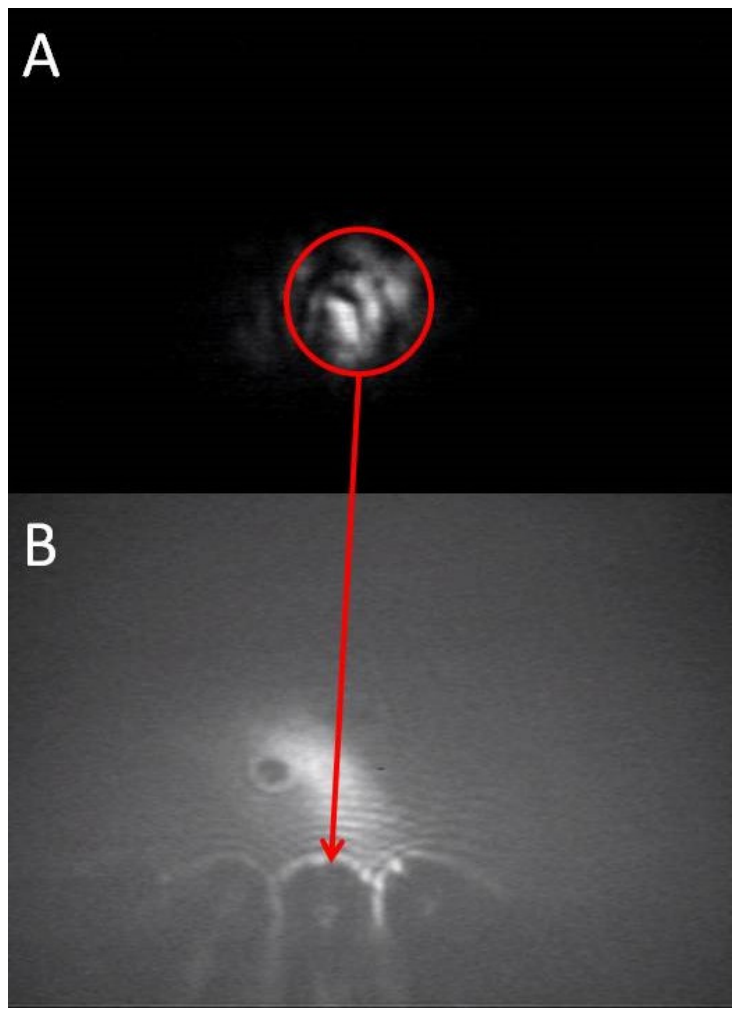

Figure 6.8: A) Output mode profile of the ULI waveguide in Cr:ZnSe at an output power of $1 \mathrm{~W}$. The photo has been scaled and resized for viewing. B) The unscaled image of the beam propagating through the bulk crystal. The waveguide structure can be seen below the beam and indicated by the red arrow.

In this case, damage occurred to the waveguide coating at an input pump power of $12.2 \mathrm{~W}$, which approximately corresponds to an intensity of $200 \mathrm{~kW} / \mathrm{cm}^{2}$ at a spot size of $80 \mu \mathrm{m}$. The difference in the specified coating damage threshold and the actual damage threshold is likely due to localized heating and differential thermal expansion of the coating and the sample. There are other techniques for creating high damage threashold coatings/configuration that could be employed in future efforts [5]. 


\subsubsection{Fe:ZnSe Results}

Utilizing the setup shown in Figure 6.4, the Fe:ZnSe sample was aligned to an erbium fiber laser operating at $2.94 \mu \mathrm{m}$ (Coractive ILM series) capable of $2 \mathrm{~W}$ of output power. The erbium fiber output was focused into the $80 \mu \mathrm{m}$ diameter Fe:ZnSe waveguide using a $4 \mathrm{~cm}$ focal length lens, which produced a spot size of $\approx 50 \mu \mathrm{m}$. A maximum output power of $995 \mathrm{~mW}$ was obtained at $1.9 \mathrm{~W}$ of input pump power producing a slope efficiency of $58 \%$, Figure 6.10 . The laser exhibited single mode output shown in the inset picture of Figure 6.10 with a $1 / e^{2}$ diameter of approximately $70 \mu m$ measured by a 90/10 knife edge measurement.

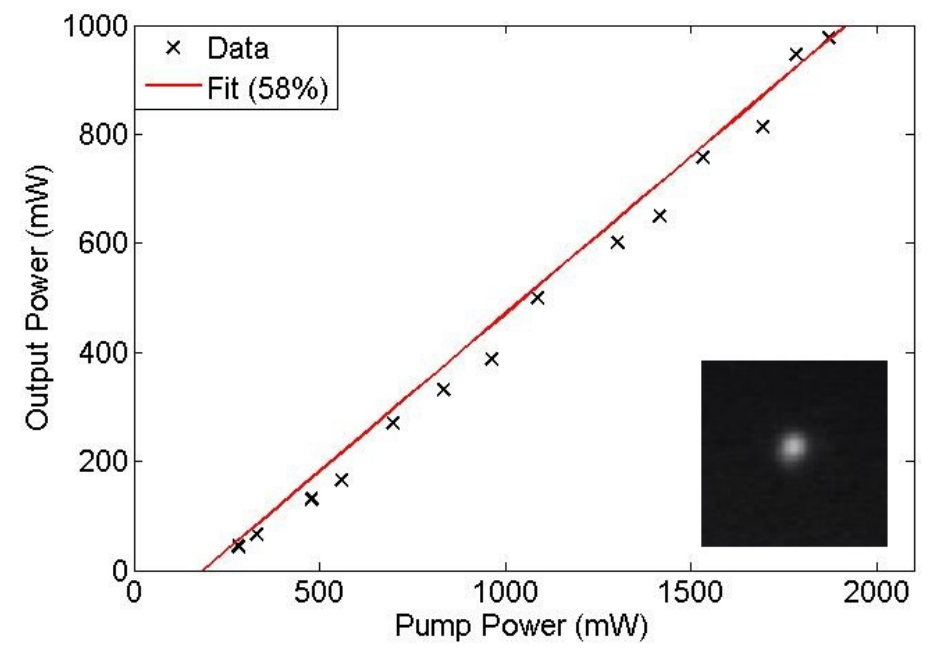

Figure 6.10: Slope efficiency obtained from the Fe:ZnSe waveguide laser. Inset picture shows the output mode of the Fe:ZnSe waveguide laser.

The output spectra of the Fe:ZnSe waveguide laser can be seen in Figure 6.11. The output of the laser was centered at $4070 \mathrm{~nm}$ with a spectral bandwidth of $70 \mathrm{~nm}$. The spectral output of this laser is broader than previously demonstrated Fe:ZnSe waveguide lasers [31]. This is likely due 
to decreased interaction with the cladding structure in this device as compared to previous devices. There is a general trend when moving towards larger diameter waveguides, that the spectral output of the laser broadens. This has been shown to occur in Cr:ZnSe lasers, moving from $2 \mathrm{~nm}$ [69] to $10 \mathrm{~nm}$ [30], and even greater in this work $(20 \mathrm{~nm})$, which corresponds to the largest diameter Cr:ZnSe waveguide laser. In addition, Fe:ZnSe was initially demonstrated with an asymmetric $151 \mu m \times 40 \mu m$ [31] cladding profile, which produced output spectra with a FWHM of $10 \mathrm{~nm}$. In this work, the cladding profile was increased to an $80 \mu m$ diameter quasi-symmetric profile, which increased the spectral bandwidth to $70 \mathrm{~nm}$. The Fe:ZnSe waveguide exhibited increased slope efficiency compared to the Cr:ZnSe waveguide. The increased performance is due to the increased upper-state lifetime of the Fe:ZnSe sample, which increases the gain of the system. Additionally, the longer wavelength laser emission reduces scattering losses proportional to $1 / \lambda^{4}$ and decreased waveguide diameter allow for increased overlap between the pump mode and the laser mode.

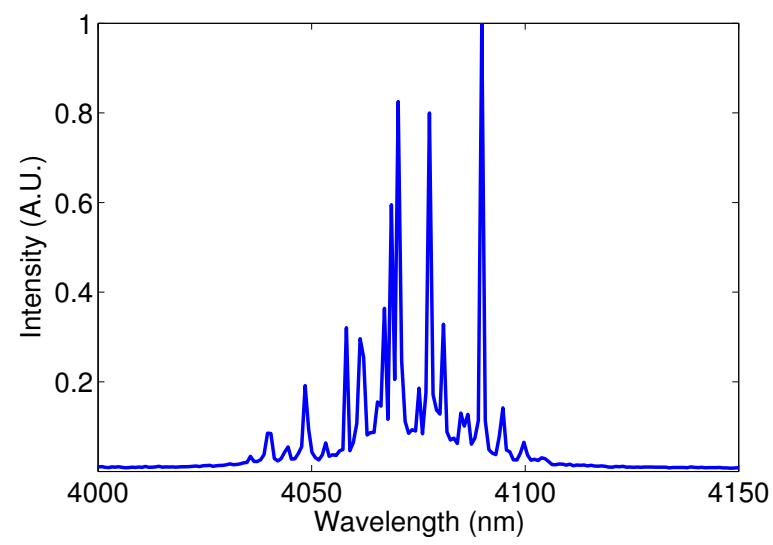

Figure 6.11: Output spectra from the Fe:ZnSe waveguide laser obtained using a Thorlabs OSA (Model OSA205). 


\subsection{Gain Switching}

In addition to pure $\mathrm{CW}$ operation, pulsed lasers in $\mathrm{Cr}: \mathrm{ZnSe}$ were also tested. Pulsed laser sources have a few key difference from CW sources, which make them ideal for specific application. Pulsed lasers have the ability to reach peak powers hundreds to thousands of times greater than the peak power obtainable from $\mathrm{CW}$ lasers. Nonlinear processes are all intensity driven mechanisms, then pulsed laser sources are usually required for efficient operation.

\subsubsection{Fabrication}

Again, guided-wave structures were fabricated in Cr:ZnSe using ultrafast laser inscription(ULI). The waveguide structures were directly written into an $8.5 \mathrm{~mm} \times 6.5 \mathrm{~mm} \times 2.1 \mathrm{~mm}(L \times W \times H)$ polycrystalline $\mathrm{Cr}: \mathrm{ZnSe}$ sample doped to $7 \times 10^{18} \mathrm{~cm}^{-3}$ and with waveguide propagation along the $8.5 \mathrm{~mm}$ length of the sample. The parameters used for inscription of these waveguides were $750 \mathrm{fs}$ pulses at $100 \mathrm{kHz}$ repetition rate. The beam was focused using a 0.68 NA objective. The waveguide was made up of several modified regions which were inscribed transversely to the direction of propagation of the waveguide. Each modified region was translated several times while inscribing the same region, often referred to multi-scan or overscans, which produces a reduction in refractive index. The decrease in refractive index of the tubular cladding constrains the guided mode to the core of the waveguide structure. The cross section of the inscribed waveguide can be seen in Figure 6.12. The dark regions are the modified cladding of the waveguide while the core remains unmodified. 


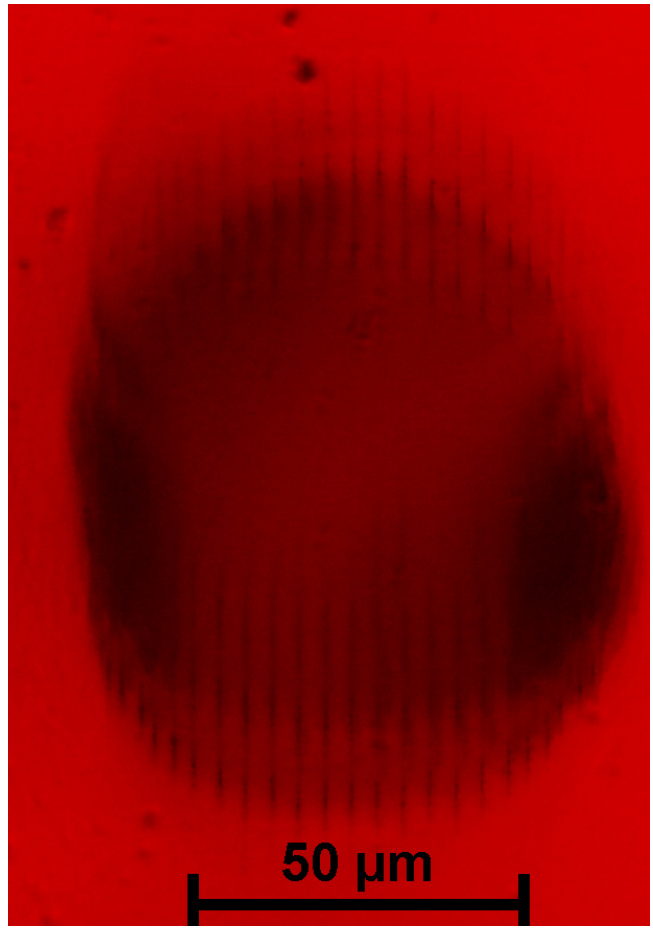

Figure 6.12: Inscribed waveguide structure showing modified cladding and unmodified waveguide core. The picture was taken looking into the direction of propagation of the waveguide. The waveguide core was measured from the edges of the modified region.

An end-pumped, Q-switched Ho:YAG laser was constructed, similar to the work presented previously [83, 84], was used to deliver pulsed output for gain-switched pumping of the Cr:ZnSe waveguide laser. This laser was capable of producing $5.5 \mathrm{~W}$ of output at $2.09 \mu \mathrm{m}$ with variable pulse widths from $400 \mathrm{~ns}$ to $60 \mathrm{~ns}$ and repetition rates from $50 \mathrm{kHz}$ to $500 \mathrm{~Hz}$. For this experiment, the laser was run at a repetition frequency of $15 \mathrm{kHz}$, with a pulse width of $\approx 400 \mathrm{~ns}$, in order to minimize the risk of coating damage. 


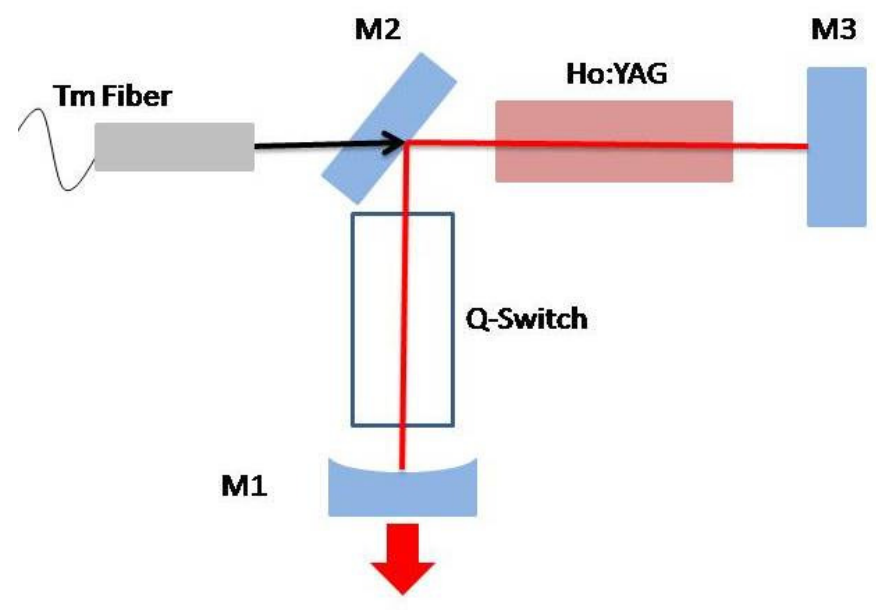

Figure 6.13: Ho:YAG laser setup.

The waveguide sample was AR coated on both ends for $1.9-3 \mu \mathrm{m}$. The sample was then affixed to a cooling block with thermal paste. The cooling block was capable of pitch and yaw adjustment along with translation (horizontal and vertical) perpendicular to the propagation direction. Figure 6.14 shows a diagram of the completed laser cavity where M1 was the incoupler (AR $2.1 \mu m$, HR $2.3-3 \mu m$ ), M2 was the outcoupler (variable precentage $2.3-3 \mu m$, AR $2.1 \mu m$ ), L1 and L2 were focusing and collimating optics and DI was a dichroic splitting mirror. L1 was a $5 \mathrm{~cm}$ focal length lens which produced a spot size of approximately $50 \mu \mathrm{m}$ diameter at the focal plane. The distance from L1 to the front of the sample was adjusted to maximize the output power from the waveguide. The waveguide core was approximately $40 \mu \mathrm{m}$ diameter. 


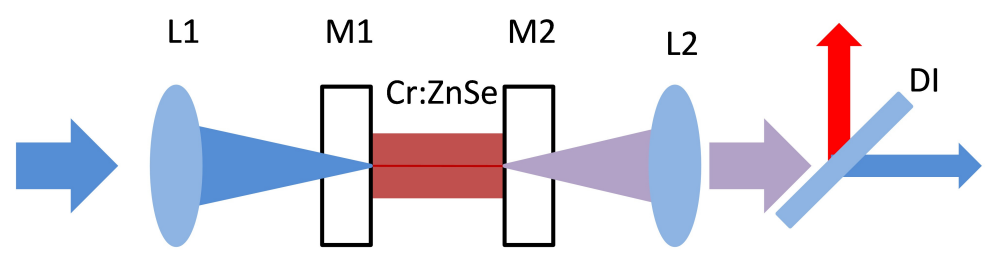

Figure 6.14: Experimental setup of the Cr:ZnSe pulsed waveguide laser, with cavity mirrors placed directly against the waveguide sample.

\subsubsection{Results}

Pulsed pumping utilizing the Ho:YAG laser produced pulsed lasing from the waveguide structure. In order to measure the power and pulse shape, two dichroic mirrors were used to separate the pump pulse from the chromium laser output pulse. The dichroic mirrors reflected $\approx 1 \%$ of the pump pulse. Thus, at full power, only $300 \mu W(20 n J)$ of pump energy was present at the detector. The output pulse shape closely followed the input pulse shape. Figure 6.15 shows the pump and output pulses. The pulses were normalized in energy such that the area under each pulse is proportional to the pump and output pulse energy. The pump and laser output pulses were fitted to a Gaussian pulse shape. The fitted FWHM of the Gaussian pulses were $402 n s$ for the output beam and $468 n s$ for the pump beam. 


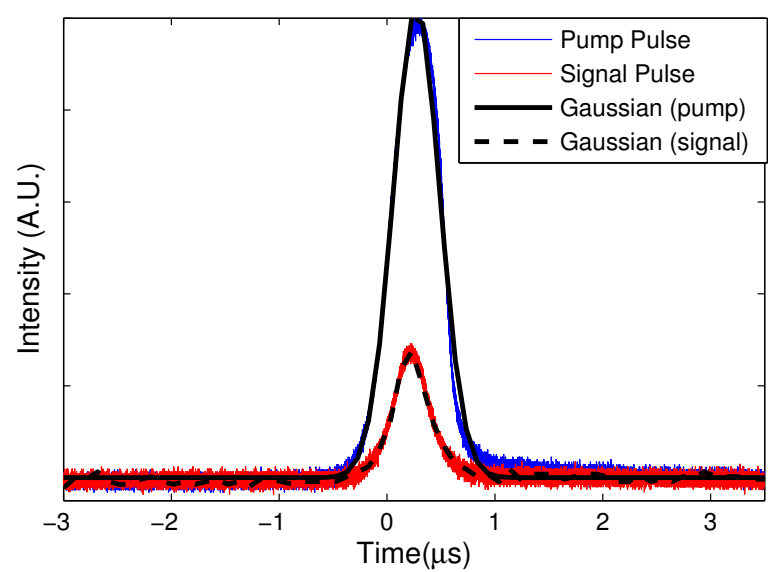

Figure 6.15: Normalized temporal output of the pump pulse(blue) and the signal output pulse(red). The pump and signal pulses were synchronized for comparison purposes. Unsynchronized pulses lead to a slight shift between pump and signal pulses due to the pulse buildup time of the resonator. However, the time shift between pump and signal pulses is small due to the short cavity length.

Average power of the pulsed pump laser was kept under $3 \mathrm{~W}$ as a precaution to prevent coating damage. The coatings were rated to have a damage threshold of $15 \mathrm{~J} / \mathrm{cm}^{2}$. At $250 \mu \mathrm{J}$ per pulse at a $50 \mu \mathrm{m}$ spot size, a fluence of $12.7 \mathrm{~J} / \mathrm{cm}^{2}$ was produced.

Figure 6.16 shows the slope efficiency of the waveguide laser with no signs of thermal rolloff at the highest pump power used $(3.3 \mathrm{~W})$. Based on calculations presented in Berry et al.[30], we would not expect to observe any thermal effects. Furthermore, the reduced duty cycle of pulse operation aids in the reduction of the thermal load on the system allowing thermal energy to dissipate between pulses. Using a 70\% reflective outcoupler, a maximum output energy of $12.1 \mu \mathrm{J}$ was achieved at $155 \mu \mathrm{J}$ of input energy producing a slope efficiency of $7.6 \%$ and an average power of $182 \mathrm{~mW}$. A $50 \%$ outcoupler was also tested in the same configuration. The $50 \%$ outcoupler enabled a maximum output energy of $10 \mu J$ at $112 \mu J$ of input energy. The $50 \%$ outcoupler yielded a slightly higher slope efficiency at $9.8 \%$. The threshold for lasing was approximately $7 \mu J$ for the $70 \%$ outcoupler and $19 \mu \mathrm{J}$ for the $50 \%$ outcoupler. The slope efficiencies in Figure 6.16 
were calculated using the absorbed energy in the crystal as the input energy. The error bars in Figure 6.16 were calculated using differential error analysis of the PRF (pulse repetition frequency) of both the pump and signal lasers. Using a LeCroy WaveRunner oscilliscope and an extended range InGaAs detector, the pump and signal pulses were observed. Taking advantage of the pulse arrival statistics, the standard deviation of the PRF was calculated. The PRF standard deviation and the average power standard deviation can then be used along with the mean PRF and average power to generate the measurement error. Simultaneous measurement of the input and output pulses revealed that the main contributing factor to the output pulse variation was fluctuations in the input pulses.

Previously demonstrated CW Cr:ZnSe waveguide lasers had slope efficiencies of 2.5\%[69], $21 \%[30]$, and $41 \%$ [29]. However, the absorption cross section of Cr:ZnSe at $2.1 \mu m$ is a factor of 4 smaller [4] than at $1.908 \mu m$ [30] or $1.928 \mu m$ [72] pump wavelengths used for CW Cr:ZnSe waveguide laser demonstrations. With a factor of 4 decrease in the absorption cross-section, $35 \%$ of the $2.1 \mu \mathrm{m}$ pump light incident on the front face of the $8.5 \mathrm{~mm}$ long Cr:ZnSe laser crystal was measured to be transmitted. The decreased absorption at $2.1 \mu \mathrm{m}$, as compared to $1.908 \mu \mathrm{m}$, will produce a decrease in slope efficiency. The incoupling and outcoupling losses from the cavity optics were approximately $10 \%$ and it can be assumed that waveguide losses were comparable to the previously published value of $0.7 \mathrm{~dB} / \mathrm{cm}$ [69]. Additionally, waveguide insertion losses could also cause decreased efficiency. The best performing pulsed waveguide had a $40 \mu \mathrm{m}$ core, which has a different NA than the CW waveguides. Performance could be improved by using a longer crystal, increasing the $\mathrm{Cr}^{2+}$ doping concentration, or changing to a shorter wavelength, pulsed, pump laser. Reduction of passive losses through improved waveguide fabrication, optimized mirror coatings and optimized mode overlap can also lead to improved lasing efficiency. 

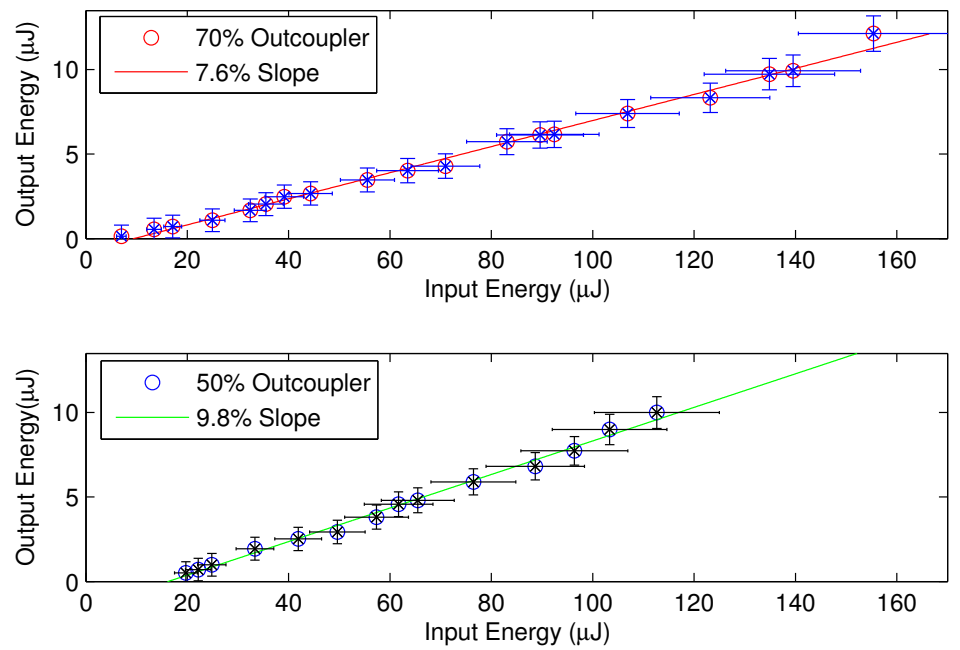

Figure 6.16: Slope efficiency of the Cr:ZnSe waveguide laser pumped by a pulsed Ho:YAG laser. The error bars represent pulse to pulse variations in the absorbed and output energies.

The output of the laser was directed into an Acton SP2750 monochromator, utilizing an SRS 830 lock-in amplifier to detect only signals with a $15 \mathrm{kHz}$ modulation, in order to investigate the output spectrum of the laser. The instrument utilized a $300 \mathrm{~g} / \mathrm{mm}$ grating blazed at $2 \mu \mathrm{m}$ with monochromator slit widths of $50 \mu \mathrm{m}$ producing a spectral resolution of $0.2 \mathrm{~nm}$. The laser output was centered around $2492 \mathrm{~nm}$ with a spectral width (FWHM) of $0.7 \mathrm{~nm}$ (see Figure 6.17). The output spectrum is narrower than the continuous-wave free-running output spectrum, which is typically between $10-50 \mathrm{~nm}$ wide[73]. The cause of the spectral narrowing is unknown but the waveguide structure demonstrates a wavelength selective characteristic which was also observed in the work of Macdonald et al.[69]. Both experiments used similar setups, which could cause both to observe the narrowing effect. As noted in chapter II, the inscription process produces HIP like conditions, where it has been shown to convert inhomogeneously broadened laser material into homogeneously broadened material. The increased interaction with HIP-like material could be a 
reason why the devices operate with a narrow output bandwidth. In general, it has been observed that smaller waveguides produce narrower output bandwidths from the laser.

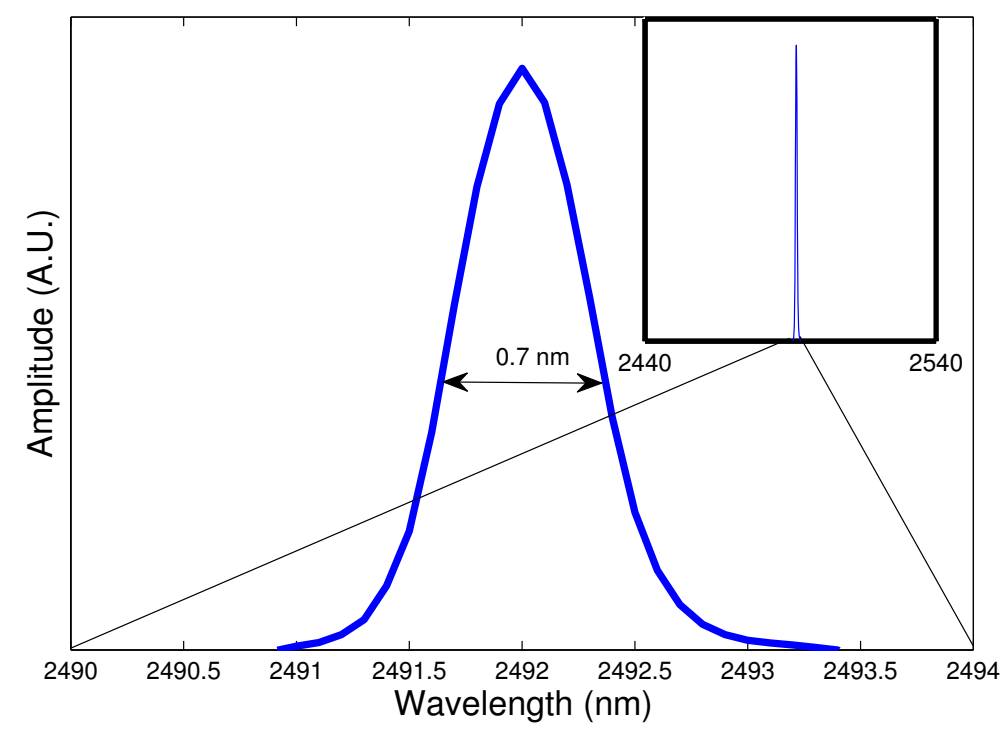

Figure 6.17: Output spectra of the Cr:ZnSe waveguide laser centered around $2492 \mathrm{~nm}$.

Utilizing a $7.5 \mathrm{~cm}$ lens to focus the output beam, an $\mathrm{M}^{2}$ measurement was performed on the laser output using a 90/10 knife-edge scan. The beam quality measurement, $\mathbf{M}^{2}$, was found by a least squares fit to the experimental data using

$$
w(z)=w_{0} \cdot \sqrt{1+M^{2}\left(\frac{z-z_{0}}{z_{R}}\right)^{2}}
$$

where $z_{R}$ is the Rayleigh range of the beam, $w_{0}$ is the beamwaist radius, $z$ is the distance from the lens and $z_{0}$ is the location of the beamwaist. An $\mathrm{M}^{2}$ value of 6.22 for the x-direction and 5.86 for the y-direction was found to fit the data from the output beam, which can be seen in Figure 6.18. Due to the non-optimal fit to Figure 6.18 , an $\mathrm{M}^{2}$ of 7 is best used as a qualitative measure of the multi-mode nature of the guided mode. 

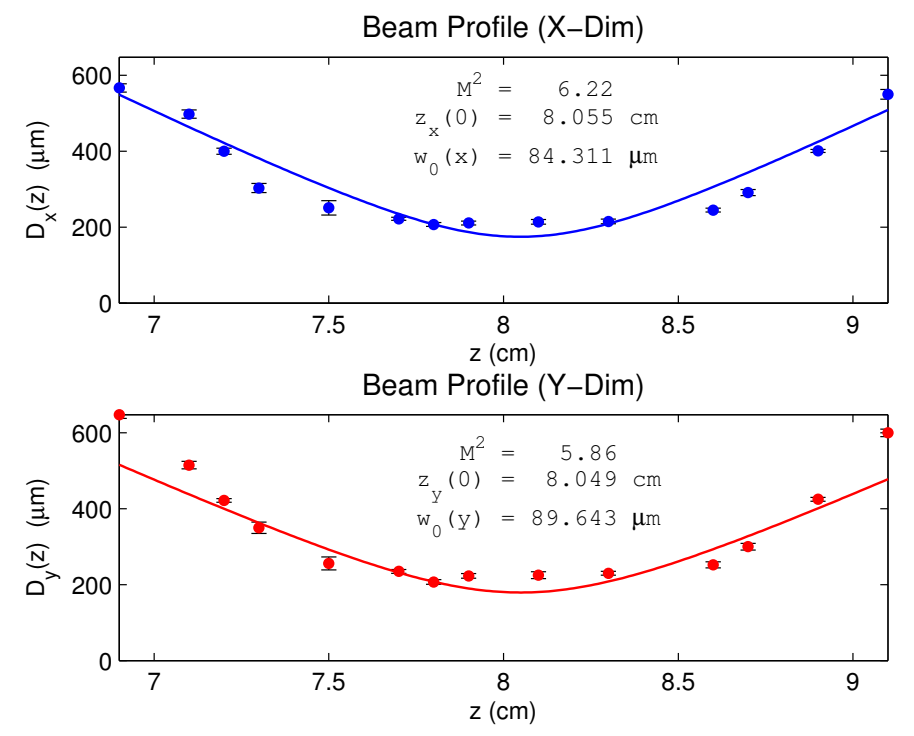

Figure 6.18: $\mathrm{M}^{2}$ measurement of the pulsed waveguide output using 90/10 knife edge scan.

The $\mathrm{M}^{2}$ value indicates multimode operation of the waveguide laser. The waveguide was sufficiently large to support multimode operation at the lasing wavelength. An Electrophysics PV320 camera was used to image the output of the waveguide (Figure 6.19), which shows the multimode transverse output profile. Additionally, Figure 6.19 shows the case of a misaligned waveguide. In the misaligned configuration, no lasing action was observed from the device. 


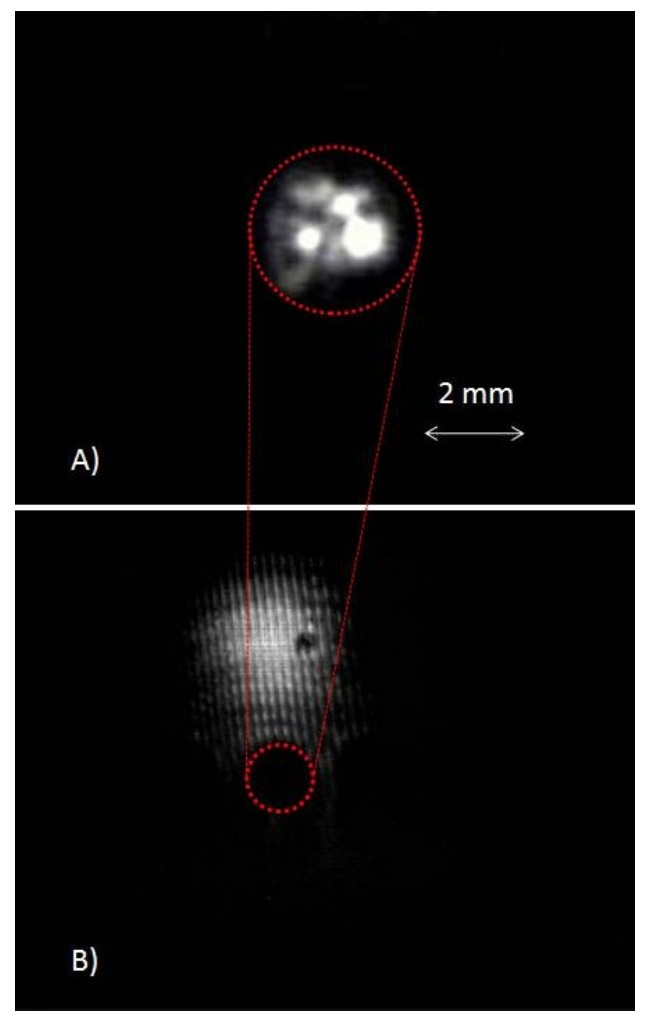

Figure 6.19: A) Output mode shape measured by a Electrophysics PV320 camera after collimation and $30 \mathrm{~cm}$ of propagation. B) Image of the vertically translated waveguide structure showing the unguided pump beam.

\subsection{Bragg Waveguides}

The principal of operation of a Bragg reflector is that for the desired wavelength, the optical path length difference between reflections at subsequent interfaces is half of the wavelength. The reflection components interfere constructively to produce a strong reflection of which the reflectivity is determined by the length of the grating and the refractive index contrast. The basic operation of a Bragg grating can be seen in Figure 6.20. Generation of Ultrafast Laser Inscribed (ULI) gratings have been shown using highly complex techniques that rely on synchronization of an acousto-optic modulator and stage movements [85, 86, 87]. 


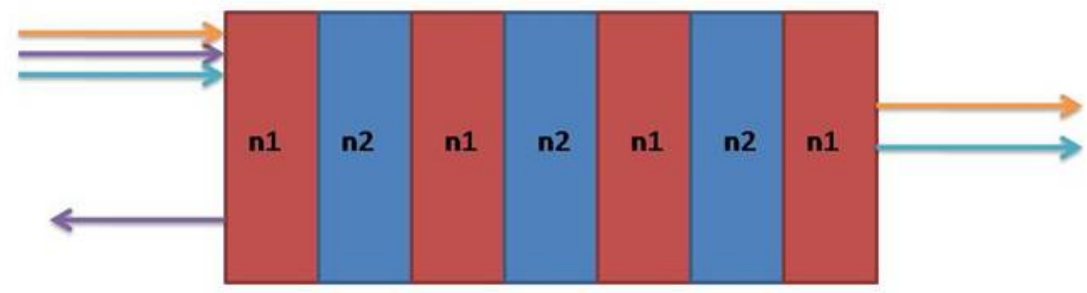

Figure 6.20: Bragg reflector for reflecting a single wavelength from several wavelengths.

A more simplistic approach would be to directly write the Bragg grating by varying the movement of the stage while traveling in a helical pattern, Figure 6.21. In Figure 6.21, the sample is translated with periodically varying speed in the z-direction while the sample moves in a circular pattern in the $\mathrm{x}$ and $\mathrm{y}$-direction. In theory, this technique could be used for a large variety of Bragg structures including chirped gratings, long period (high order) Bragg gratings and multi-period Bragg gratings. 


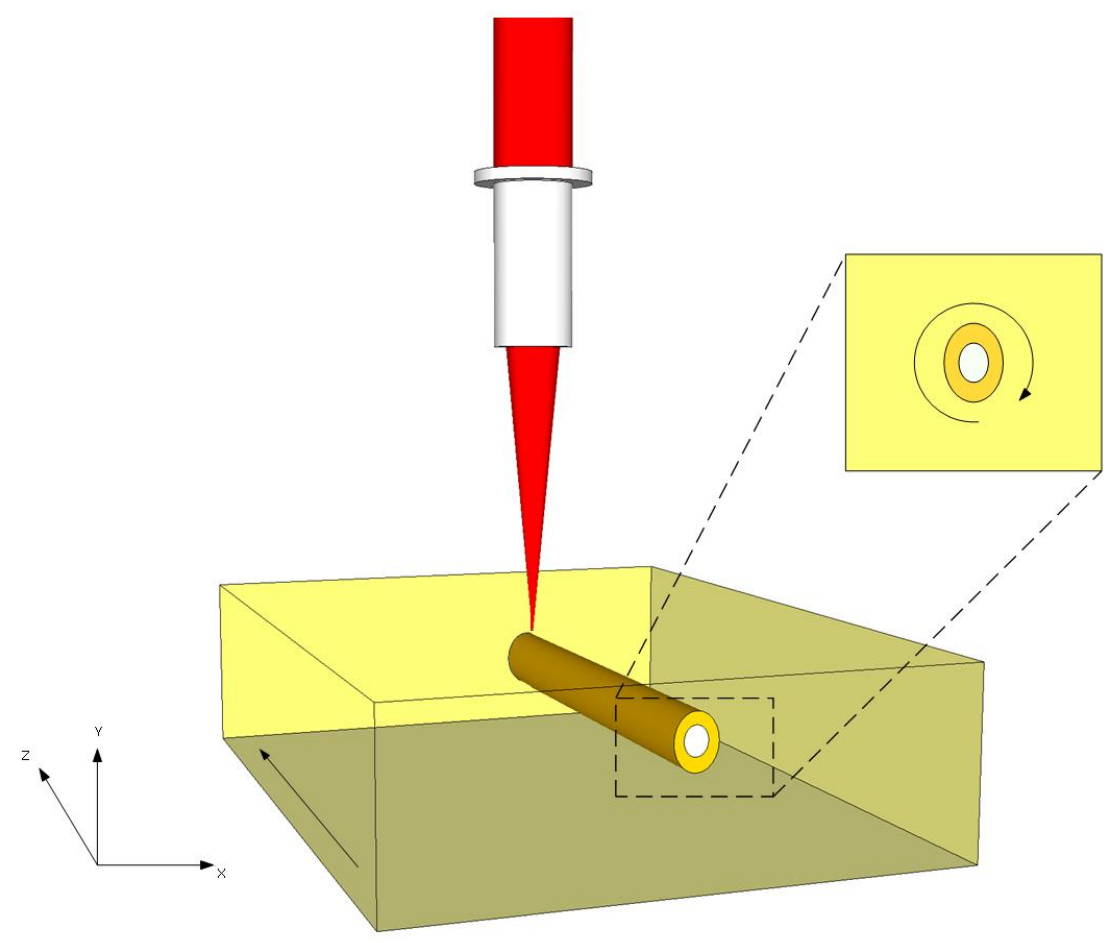

Figure 6.21: Helical inscription of waveguide structures

From Figure 6.22, it can be seen that a Bragg reflector can be used as a mirror to reflect a certain wavelength. Extrapolating this idea, it can be utilized to create a laser cavity out of two Bragg reflectors and a gain section. A visual interpretation of this idea is seen in Figure 6 with the dark red sections being the grating and the two light red sections representing a waveguide structure in the material. In addition, the Bragg structure can be utilized throughout the length of the waveguide to produce a distributed feedback (DBR) type structure. 


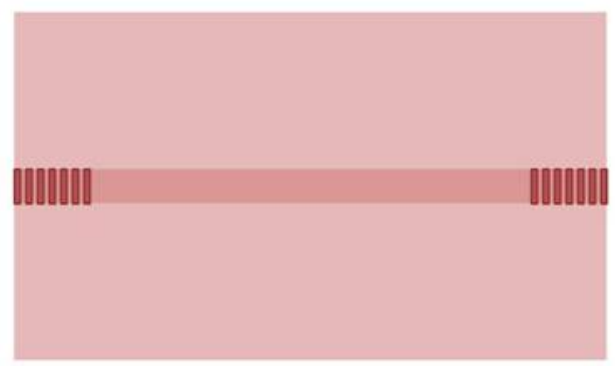

Figure 6.22: Bulk sample of Cr:ZnSe with Bragg reflectors on either side of the waveguide structure.

By varying the inscription speed, periodic changes in the effective index of the waveguide can be obtained. An example of this index change can be seen in . Figure 6.23 shows an example of the waveguide produced by helical inscription in $\mathrm{ZnSe}$. This process can be duplicated in doped materials such as Cr:ZnSe and Fe:ZnSe.

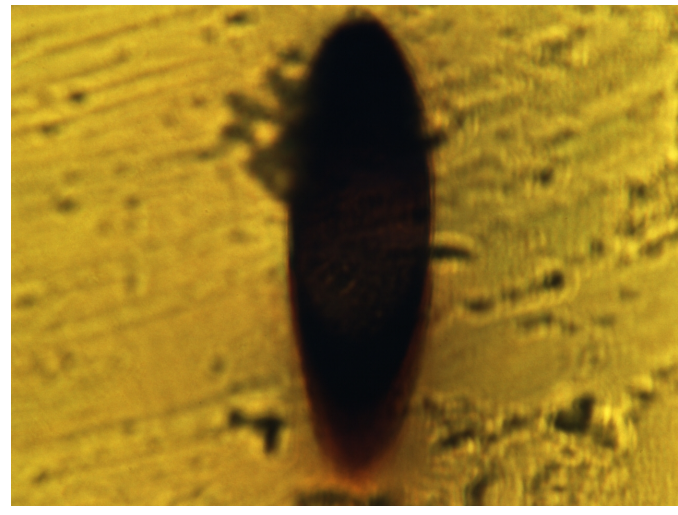

(a) Propagation direction

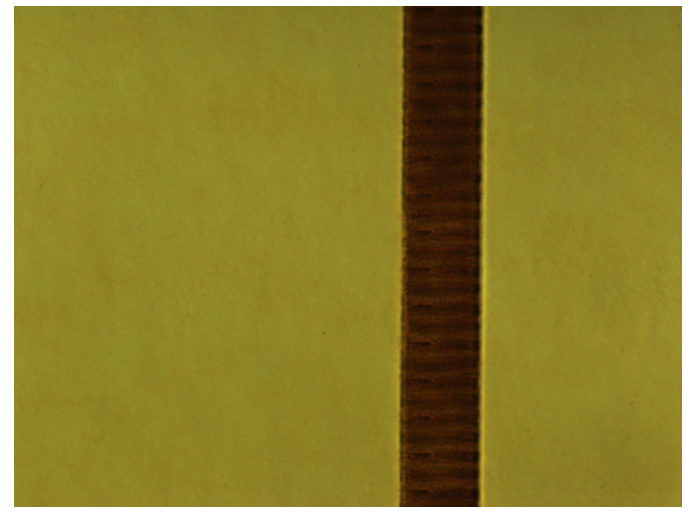

(b) Top down

Figure 6.23: Example of Bragg structures inscribed in ZnSe. (a) Endface image of the helical waveguide (b) Top-down image of the helical waveguide.

The structure shown in Figure 6.23 was created in ZnSe with the parameters shown in Table 6.4. In addition, the Bragg structuring was created using a 8th order grating, found from the following 
equation, $\Lambda=m \lambda /\left(2 n_{e f f}\right)$. In this equation, $\Lambda$ is the grating period, $\mathrm{m}$ is the integer order of the grating, $\lambda$ is the wavelength of light, and $n_{e f f}$ is the effective index of the waveguide. For this case, $\Lambda$ was calculated to be approximately $4 \mu \mathrm{m}$. An 8 th order grating spacing was chosen because the inscription laser can only produce feature sizes on the order of $1 \mu \mathrm{m}$. It should be noted that the reflectivity of the Bragg grating will decrease for higher order grating periods, but the overall length of the device can be increased to compensate for the drop in reflectivity.

Table 6.4: Parameters used for inscription of Bragg structured cladding waveguides.

\begin{tabular}{|c|c|}
\hline Power & $110 \mathrm{~mW}$ \\
\hline Translation Speed & $10 \mathrm{~mm} / \mathrm{s}$ \\
\hline Repetition Frequency & $100 \mathrm{kHz}$ \\
\hline Pulse Width & $800 \mathrm{fs}$ \\
\hline
\end{tabular}

The waveguides shown in Figure 6.23 were reproduced in a sample of $\mathrm{Cr}: \mathrm{ZnSe}$. The sample had waveguides with both Bragg structures and without Bragg structures to allow for direct comparison of the effects of the periodic structuring. Additionally, input and output optics were directly coated onto the end facets of the sample The incoupler coating was AR at $1.9 \mu m$ to $2.1 \mu m$ and HR from $2.3 \mu \mathrm{m}$ to $3 \mu \mathrm{m}$. The outcoupler coating was AR at $1.9 \mu \mathrm{m}$ to $2.1 \mu \mathrm{m}$ and $70 \%$ reflective from $2.3 \mu \mathrm{m}$ to $3 \mu \mathrm{m}$.The setup utilized is shown in Figure 6.24, where L1 and L2 were focusing and collimating lenses with focal lengths of $5 \mathrm{~cm}$. 


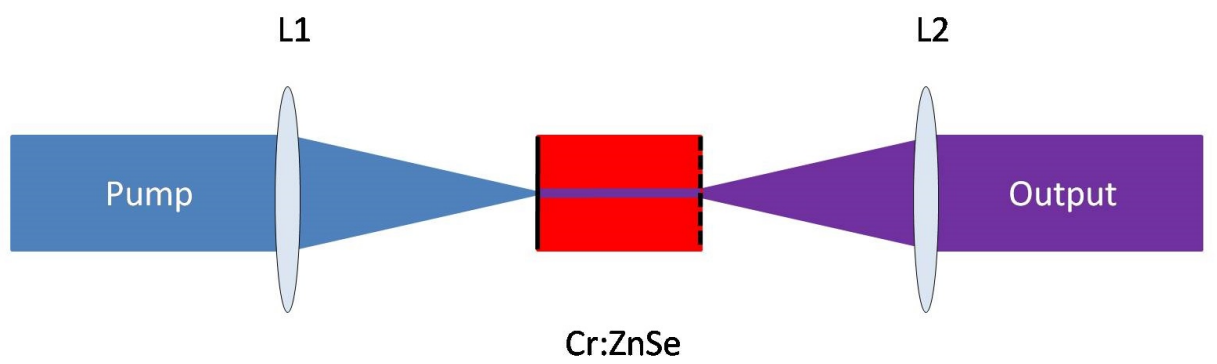

Figure 6.24: Experimental setup for testing of the Bragg structured waveguides.

Preliminary testing of the waveguides shows that the Bragg structuring does have an effect on the output of the waveguide, Figure 6.25. The top graph in Figure 6.25 shows the theoretical reflectivity spectrum of a multimode waveguide with supported modes varying from $n_{\text {eff }}=2.44$ to $n_{\text {eff }}=2.37$. The bottom graph in Figure 4 shows the output spectrum from two identical waveguides. The only difference between the two waveguides is that one was structured with a varying refractive index profile similar to that seen in Figure 6.23. 

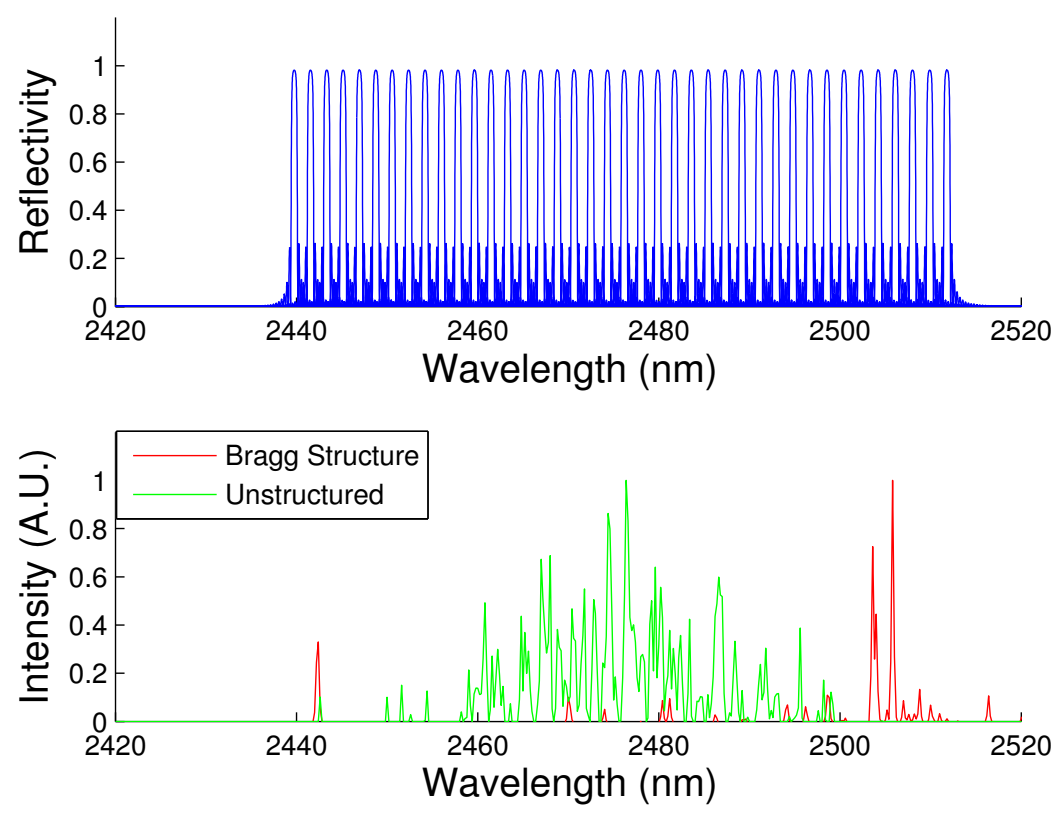

Figure 6.25: Top: Theoretical reflectivity a waveguide Bragg grating with 40 evenly spaced, supported modes. Bottom: Output spectrum of identical waveguides with and without Bragg structuring.

The results in Figure 6.25 show potential for this technique to produce quality single frequency laser sources. However, there are still a few unknowns about the Bragg structuring, such as index difference between the high and low index areas and the exact number of supported modes. 


\section{CHAPTER VII}

\section{HO:YAG ULI LASERS}

\subsection{Introduction}

Direct laser sources operating around the $2 \mu \mathrm{m}$ range have seen a recent growth in utility. $2 \mu \mathrm{m}$ laser sources share many commonalities with organic absorption lines and are widely considered "eye-safe" due to their high absorption coefficient in intraocular fluids and tissues. Ho:YAG ULI waveguides are an ideal solution for creating a compact laser source, which could be suitable for medical [88], dental [89] and as scientific laser sources [84, 90]. Typically, holmium ions in a garnet host, such as YAG, are operated in one of two regimes: either by in-band pumping of the holmium

ions $[91]{ }^{5} \mathrm{I}_{7}-{ }_{-}^{5} \mathrm{I}_{8}$ transition or by energy transfer from other rare earth ions, in a co-doped crystal [92]. For both regimes, the laser operates on the holmium ${ }^{5} \mathrm{I}_{7}-{ }^{5} \mathrm{I}_{8}$ transition producing $2.09 \mu \mathrm{m}$ radiation in a YAG crystal host at room temperature [93].

As previously discussed, guided wave operation of laser sources offers several advantages to bulk lasers such as increased interaction length, compact size, and increased reliability. One approach to guided-wave operation of Ho:YAG lasers is through doping of crystalline YAG fibers $[94,95]$. However, the difficulty of producing these fibers has slowed the adaptation of this technology as a way of making laser sources. A more popularized approach is holmium doping or co-doping of holmium and thulium of ZBLAN fibers[96, 97], which is a mature technology. A maximum output of $6.6 \mathrm{~W}$ has been experimentally shown for a Ho:ZBLAN fiber [98]. Tm, Ho and 
co-doped Tm-Ho silica fiber lasers have been created with output powers well in excess of $10 \mathrm{~W}$ [99] and are commercially available. Finally, techniques such as adhesive free bonded waveguides have shown promise for producing efficient laser sources [100, 101].

Direct creation of waveguides in bulk material using ULI is an approach that was successfully used in YAG [102] and has produced low power waveguide lasers in Tm:YAG [103] and Tm:ZBLAN[104]. However, a ULI waveguide laser has not been reported in Ho:YAG previous to this work.

\subsection{Fabrication}

ULI has been shown to be a viable technique for creating waveguides inside of a variety of materials including glass [16], chalcogenides [28], and various crystalline hosts [105]. Inscription of waveguides in single crystal material can be difficult. As a starting point, test waveguides were inscribed in 0.5 at.\% holmium doped YAG sample with dimensions of $5 \mathrm{~mm} \times 5 \mathrm{~mm} \times 14 \mathrm{~mm}$. A set of twenty waveguides were inscribed at a repetition rate of $100 \mathrm{kHz}$ with an average power of $150 \mathrm{~mW}$ and a pulse width of $800 \mathrm{fs}$. The structure produced by these parameters can be seen in Figure 7.1. 


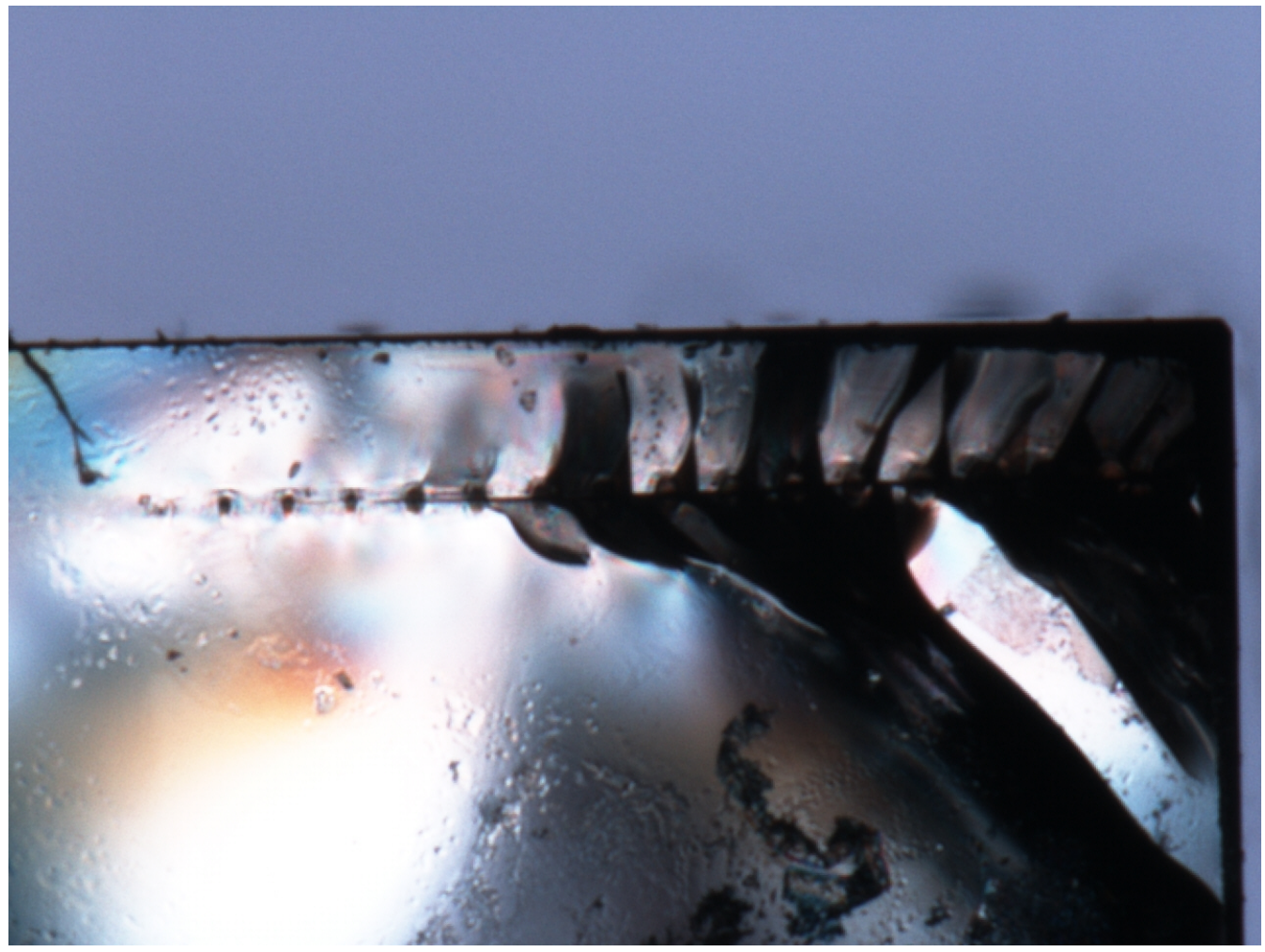

Figure 7.1: Cracking observed in Ho:YAG during initial testing of ULI parameters. The crack propagated laterally through all ULI structures, rendering the sample unusable. The picture was taken looking into the direction of propagation of the waveguide.

As can be seen from Figure 7.1, the sample experienced severe cracking. The cracking was due to high stress in the crystal. The ULI process introduces defects to the crystal structure. These defects stress the crystal, such that a stress induced break can occur. In addition, the ULI process also produces a large amount of heat at the focus of the ULI beam. The heat load coupled with the defects makes cracking an issue with waveguide creation in single crystal materials. Several other sets of waveguides were tested in the Ho:YAG sample. The parameters used and the quality of the resultant structure can be seen in Table 7.1. 
Table 7.1: Basic summary of inscription parameters and outcome of the inscription process in Ho:YAG.

\begin{tabular}{ccccc}
\hline Rep. Rate & Power & Write Speed & Pulse Width & Outcome \\
\hline $100 \mathrm{kHz}$ & $200 \mathrm{~mW}$ & $10 \mathrm{~mm} / \mathrm{s}$ & $800 \mathrm{fs}$ & crack \\
$100 \mathrm{kHz}$ & $100 \mathrm{~mW}$ & $10 \mathrm{~mm} / \mathrm{s}$ & $800 \mathrm{fs}$ & crack \\
$100 \mathrm{kHz}$ & $50 \mathrm{~mW}$ & $10 \mathrm{~mm} / \mathrm{s}$ & $600 \mathrm{fs}$ & no crack \\
$200 \mathrm{kHz}$ & $100 \mathrm{~mW}$ & $10 \mathrm{~mm} / \mathrm{s}$ & $600 \mathrm{fs}$ & crack \\
$200 \mathrm{kHz}$ & $50 \mathrm{~mW}$ & $10 \mathrm{~mm} / \mathrm{s}$ & $600 \mathrm{fs}$ & no crack \\
$500 \mathrm{kHz}$ & $100 \mathrm{~mW}$ & $10 \mathrm{~mm} / \mathrm{s}$ & $600 \mathrm{fs}$ & crack \\
$500 \mathrm{kHz}$ & $50 \mathrm{~mW}$ & $10 \mathrm{~mm} / \mathrm{s}$ & $600 \mathrm{fs}$ & no crack \\
\hline
\end{tabular}

From Table 7.1, it can be seen that relatively low power inscription is needed to prevent the Ho:YAG sample from cracking. However, slightly higher power levels can be used if the overlap between each element comprising the waveguide structure is minimized (i.e. the number of filamentation regions in each waveguide). Utilizing the IMRA $\mu$ Jewel D1000, several sets of waveguides were inscribed in a 0.5 at.\% holmium doped YAG sample. The Ho:YAG sample had dimensions of $5 \mathrm{~mm} \times 5 \mathrm{~mm} \times 14 \mathrm{~mm}$. The waveguides were inscribed at two different repetition frequencies: $100 \mathrm{kHz}$ for the multimode waveguides and $500 \mathrm{kHz}$ for the single mode waveguides. Pulse energies of $1.2 \mu J$ were used to inscribe the multimode waveguides, while pulse energies of $300 \mathrm{~nJ}$ were used for the single mode waveguides. In addition, both sets of waveguides were inscribed using a $0.68 \mathrm{NA}$ lens with a focal length of $4.1 \mathrm{~mm}$ producing a focal spot of $1 \mu \mathrm{m}$. The sample was translated using an XYZ translation stage (Aerotech Model:A3200) moving with a maximum velocity of $10 \mathrm{~mm} / \mathrm{s}$. The resultant inscribed cladding structure for the multimode waveguides can be seen in Figure 7.2. The waveguides produced were uniform in the direction of propagation and were slightly nonuniform in the transverse directions. The nonuniformities arise from slight changes in the focusing of the beam due to the depth inside of the sample and change in the overlap with surrounding modified regions. The dark circles are damage regions created by the ULI process. 
Waveguiding occurs inside of these dark rings. Similar structures have been demonstrated previously in this work and by other authors $[28,27]$. The ULI device was designed to have a number of waveguides with different core diameters to demonstrate a wide variety of operating parameters. The core sizes varied between $30 \mu \mathrm{m}$ and $120 \mu \mathrm{m}$. As core diameter increased, the number of inscription elements also increased. For example, the $50 \mu \mathrm{m}$ was composed of 60 individual inscription elements and the $80 \mu \mathrm{m}$ waveguide was composed of 80 elements. Note that as the size increases, fewer inscription elements are used to prevent cracking. The two different sets of parameters were used for creation of single and multimode waveguides respectively. The difference in the two recipes arose from creating a larger index difference between the core and the cladding, thus creating a strongly guiding waveguide. The optical propagation losses for these samples can vary from waveguide to waveguide. A maximum loss of $1.5 \mathrm{~dB} / \mathrm{cm}$ was measured using the technique outlined previously in this work.

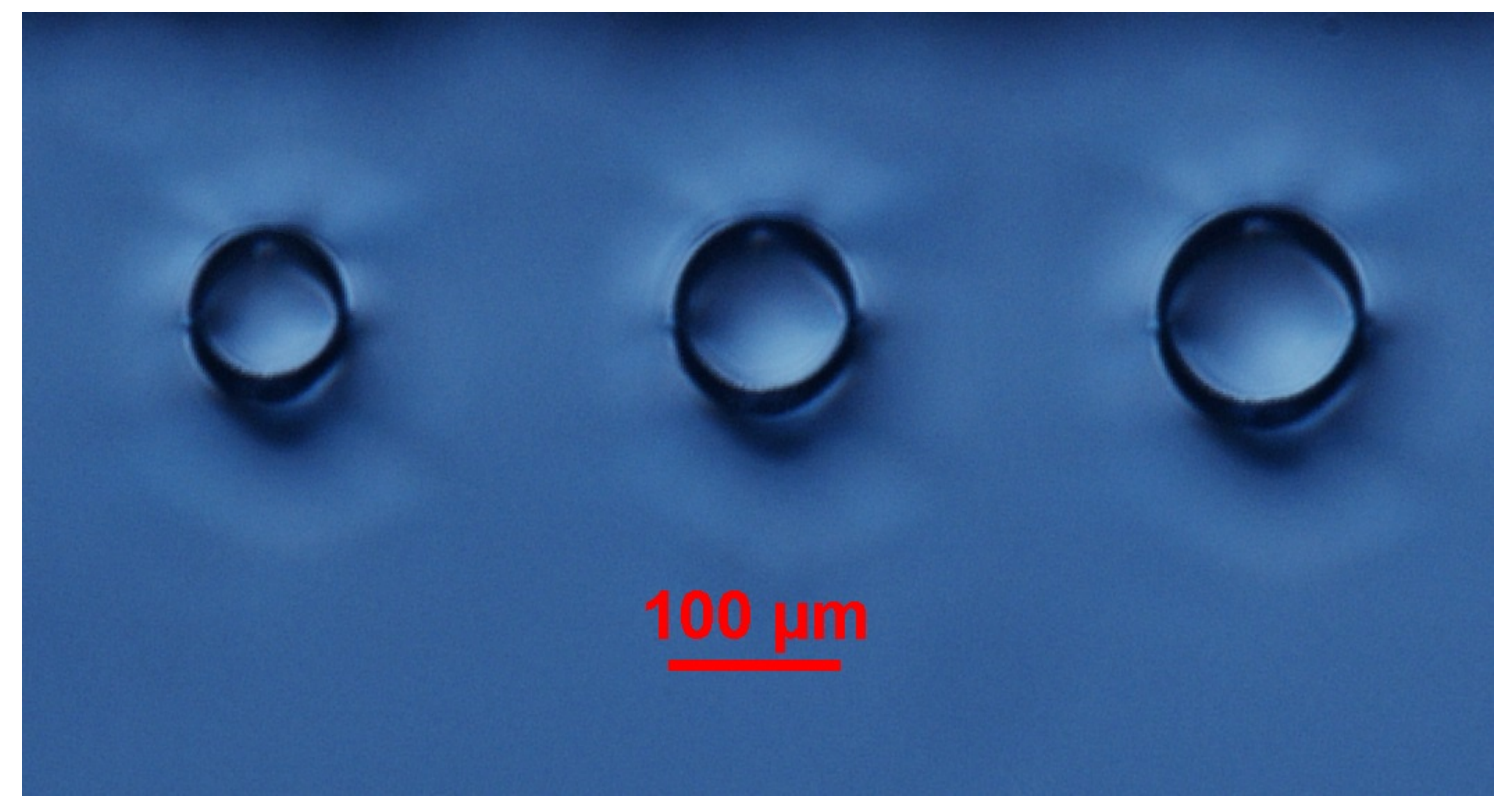

Figure 7.2: Waveguides inscribed in a 0.5 at.\% Ho:YAG sample. The picture was taken looking into the direction of propagation of the waveguide. 


\subsection{Experimental Setup}

To test the performance of the ULI waveguide devices, the waveguide sample was placed on a water cooled mount attached to a 5-axis adjustable stage. The cooling block was kept at $15^{\circ} \mathrm{C}$. Figure 7.3 shows the cavity configuration used for testing of the waveguide laser devices. Mirror M1 was AR coated for $1.9-2 \mu m$ and HR for 2.05-2.2 $\mu \mathrm{m}$. Mirror M2 was AR for $1.9-2 \mu m$ in addition to acting as the outcoupler for $2.05-2.2 \mu \mathrm{m}$. Mirror M2 was replaced with optics that had reflectivities from $30 \%$ to $97 \%$ reflective. For the setup in Figure 7.3, lenses L1 and L2 were broadband AR coated $(2-3 \mu \mathrm{m})$ with a focal length of $5 \mathrm{~cm}$. The pump source for the Ho:YAG crystal was a CW thulium fiber laser (IPG model TLR-1908-LP), which produced a maximum output power of $12 \mathrm{~W}$. Lens L1 focused the pump to a spot size of $\sim 50 \mu \mathrm{m}$, which was also used to couple the pump laser into the Ho:YAG sample. Furthermore, both entrance and exit facets of the sample were uncoated. While various waveguide sizes were manufactured and evaluated, only the best performing multimode and single mode waveguide results are presented here.

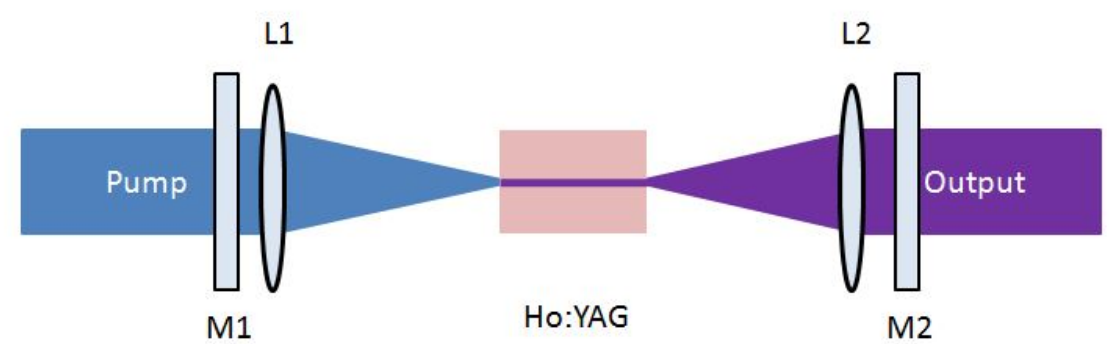

Figure 7.3: Cavity configuration used for testing of the multimode waveguides. 


\subsection{Multimode Operation}

Under in-band pumping conditions at $1.9 \mu \mathrm{m}$, the sample shown in Figure 7.2 demonstrated lasing on the ${ }^{5} \mathrm{I}_{7}-{ }^{5} \mathrm{I}_{8}$ transition. Figure 7.4 shows the laser performance of an $80 \mu \mathrm{m}$ diameter multimode waveguide. From measuring the diverging light from the waveguide, we estimate that an index difference of $\Delta n=0.01$ was obtained. This guide produced the highest output power given the pump conditions and setup shown in Figure 7.3. The slope efficiency was obtained for several output coupler reflectivities ranging from $30 \%$ to $90 \%$. It should be noted that for this measurement the pump power is recorded as the power incident on the crystal. Using a $50 \%$ reflective outcoupler, a maximum output power of $1.88 \mathrm{~W}$ with a slope efficiency of $29 \%$ was obtained. As the outcoupler reflectivities deviated from the 50\%/70\% level, output power and sloped efficiency decreased. Furthermore, the threshold powers for the $90 \%, 70 \%, 50 \%$, and $30 \%$ outcouplers were $2.1 \mathrm{~W}, 2.5$ $\mathrm{W}, 3.2 \mathrm{~W}$ and $3.6 \mathrm{~W}$ respectively. Table 7.2 reports the slope efficiency and output power obtained from each outcoupler reflectivity. At incident pump powers greater than $10 \mathrm{~W}$, thermal rollover in the output power was observed. While not investigated here, thermal rollover could be mitigated by reducing the duty cycle of the pump or by decreasing the temperature of the cooling block.

Table 7.2: Slope efficiencies and maximum output powers of Figure 7.4.

\begin{tabular}{ccc}
\hline Reflectivity & Output Power & Slope Efficiency \\
\hline $90 \%$ & $0.91 \mathrm{~W}$ & $11 \%$ \\
$70 \%$ & $1.88 \mathrm{~W}$ & $27 \%$ \\
$50 \%$ & $1.88 \mathrm{~W}$ & $29 \%$ \\
$30 \%$ & $1.12 \mathrm{~W}$ & $17 \%$ \\
\hline
\end{tabular}




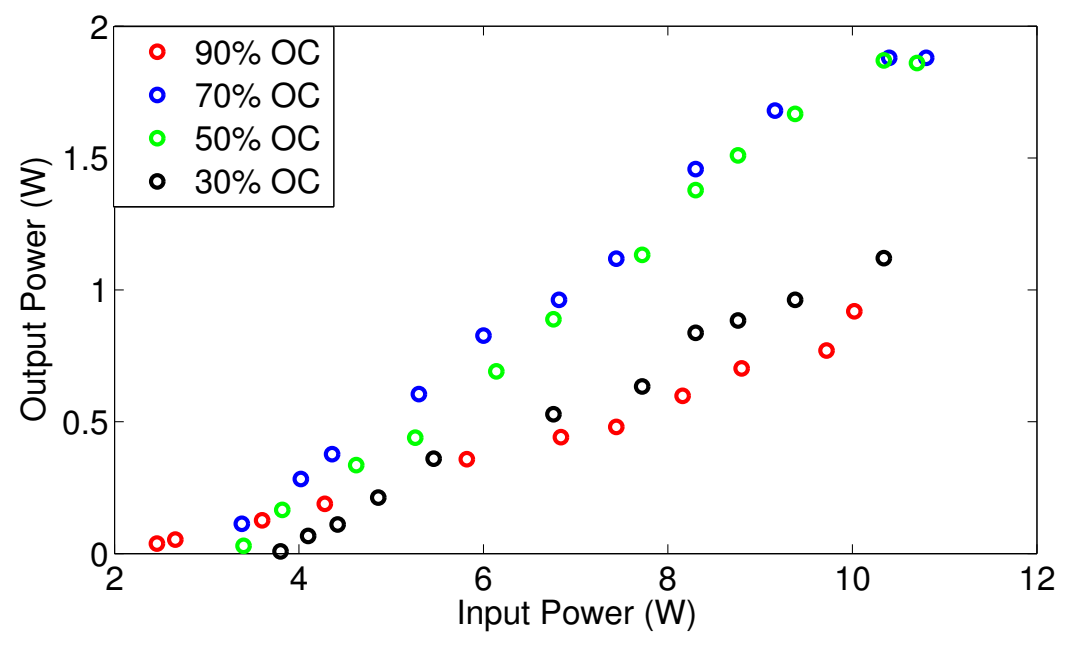

Figure 7.4: Laser performance for various output coupler reflectivities for the $80 \mu \mathrm{m}$ multimode Ho:YAG waveguide.

The spectral characteristics of the waveguide lasers were characterized using a Yokogawa optical spectrum analyzer (OSA) with a resolution of $0.02 \mathrm{~nm}$ (AQ6375). Figure 7.5 shows the spectral output of the multimode waveguides. It can be seen that the output wavelength depended on the reflectivity of the outcoupler. For the $30 \%, 50 \%$ and $70 \%$ reflective outcouplers, the output wavelength was centered at $2090 \mathrm{~nm}$. For the $90 \%$ outcoupler, the wavelength shifted to $2120 \mathrm{~nm}$. This shift is due to the increased round-trip losses associated with the lower reflectivity outcoupler and increased reabsorption losses due to overlap of the emission and absorption cross-sections [106]. The lasing wavelength shifts to the wavelength where the threshold power is the lowest, which for higher roundtrip losses, forces the laser to run at the peak of the emission cross-section at $\approx 2090 \mathrm{~nm}$. 


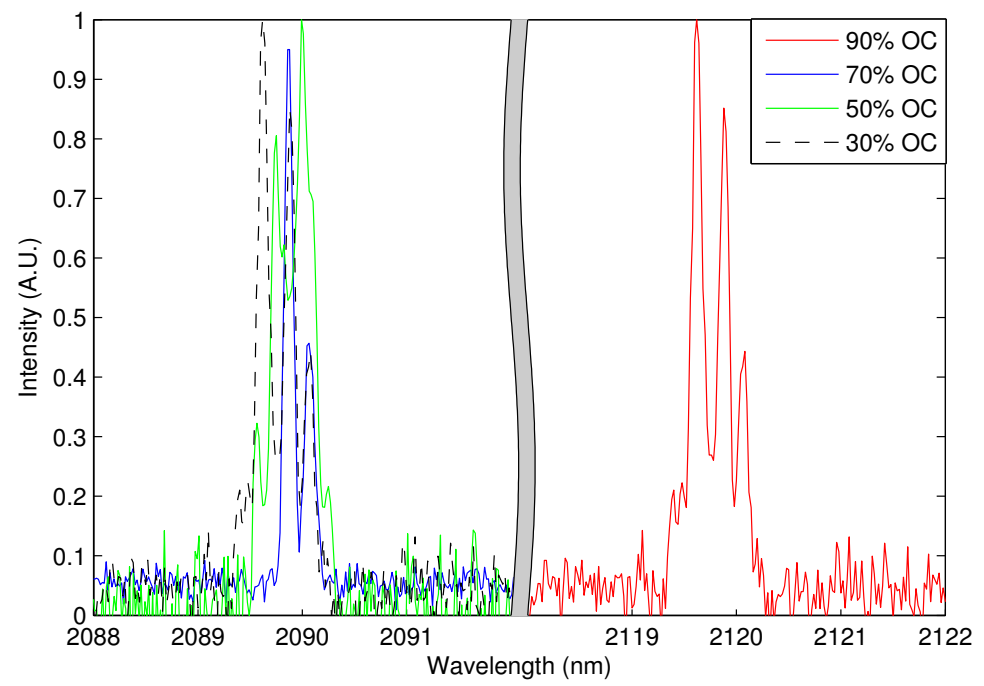

Figure 7.5: Spectral output of the $80 \mu$ m waveguide dependent on output coupler reflectivity.

\subsection{Single Mode Operation}

Single mode waveguides were tested under similar conditions as described above. The $40 \mu \mathrm{m}$ diameter waveguide was pumped using a $1.9 \mu \mathrm{m}$ thulium fiber laser. From measuring the diverging light from the waveguide, we estimate that an index difference of $\Delta n=0.001$ was obtained. Similar index differences have been observed by other authors [101] from adhesive free bonding. Figure 7.6 shows the laser performance of the single mode waveguides for various output coupler reflectivities. It can be seen that the $50 \%$ reflective outcoupler produced the best performance with a maximum output power of $440 \mathrm{~mW}$ and a slope efficiency of $16 \%$. Table 7.3 reports the results for the single mode waveguides. Using the methods previously discussed, a propagation loss of approximately $2 \mathrm{~dB} / \mathrm{cm}$ was estimated. It should be noted that other authors have shown single mode waveguides in Ho:YAG with higher efficiency and output power [107]. 


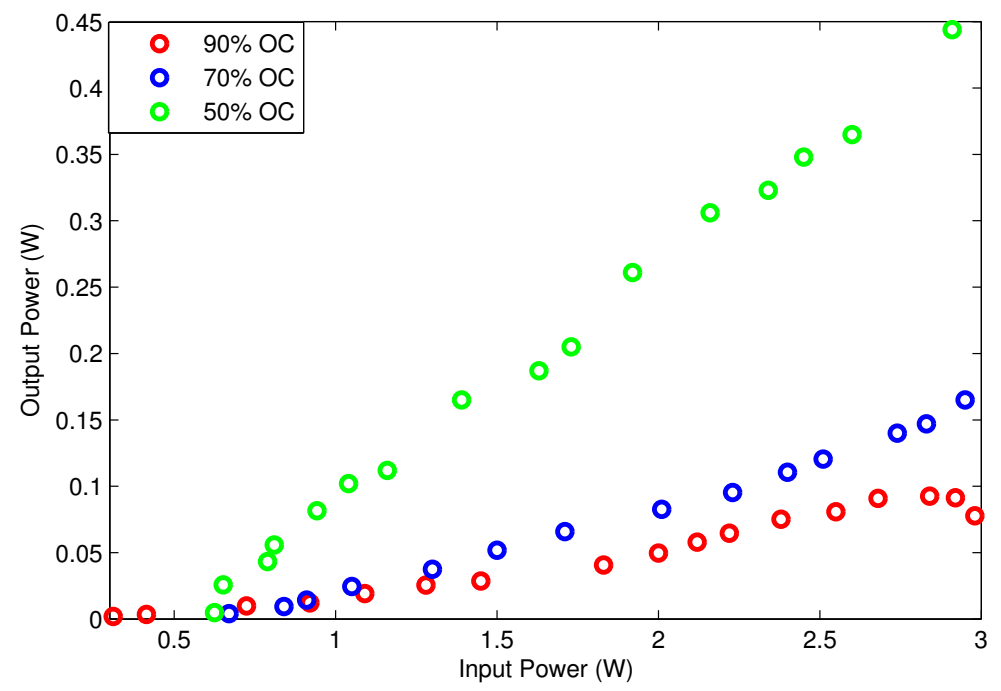

Figure 7.6: Slope efficiency for the single mode waveguides.

Additionally, the output spectra from the single mode waveguide was measured. The spectral performance was almost identical to that obtained by the multimode waveguides, with the $70 \%$ and $50 \%$ reflective outcouplers lasing at a wavelength of $2090 \mathrm{~nm}$ and the $90 \%$ reflective outcouplers lasing at $2120 \mathrm{~nm}$

Table 7.3: Slope efficiencies and output powers of Figure 7.6

\begin{tabular}{ccc}
\hline Reflectivity & Output Power & Slope Efficiency \\
\hline $90 \%$ & $92 \mathrm{~mW}$ & $3 \%$ \\
$70 \%$ & $165 \mathrm{~mW}$ & $8 \%$ \\
$50 \%$ & $444 \mathrm{~mW}$ & $16 \%$ \\
\hline
\end{tabular}

For comparison, single and multimode waveguides in Tm:YAG [103] present similar behavior to the results discussed above. In general, the multimode waveguides for both Tm and Ho:YAG exhibit higher slope efficiencies compared to the single mode waveguides. The multimode waveguides 
show promise for power scaling of these devices. However, this is at the cost of decreased mode quality.

\subsection{Mode Profiles}

The transverse mode profile of the $80 \mu \mathrm{m}$ multimode waveguide can be seen in subset picture in Figure 7.7. In order to characterize the beam quality of the laser, the $\mathrm{M}^{2}$ parameter was measured using a $10 \mathrm{~cm}$ lens to focus the output beam. The exact method used to measure and calculated the beam quality is outlined in the ISO standard 11146 . The $1 / \mathrm{e}^{2}$ beam diameter was measured at several positions and the $\mathrm{M}^{2}$, was found by a least squares fit to the experimental data using

$$
w(z)=w_{0} \cdot \sqrt{1+M^{2}\left(\frac{z-z_{0}}{z_{R}}\right)^{2}}
$$

where $z_{R}$ is the Rayleigh range of the beam, $w_{0}$ is the beamwaist radius, $z$ is the distance from the lens and $z_{0}$ is the location of the beamwaist. For the multimode waveguide laser, an $\mathrm{M}^{2}$ value of 4.9 was calculated, which can be seen in Figure 7.7. It should be noted that both vertical and horizontal directions had similar values for $\mathrm{M}^{2}$. 


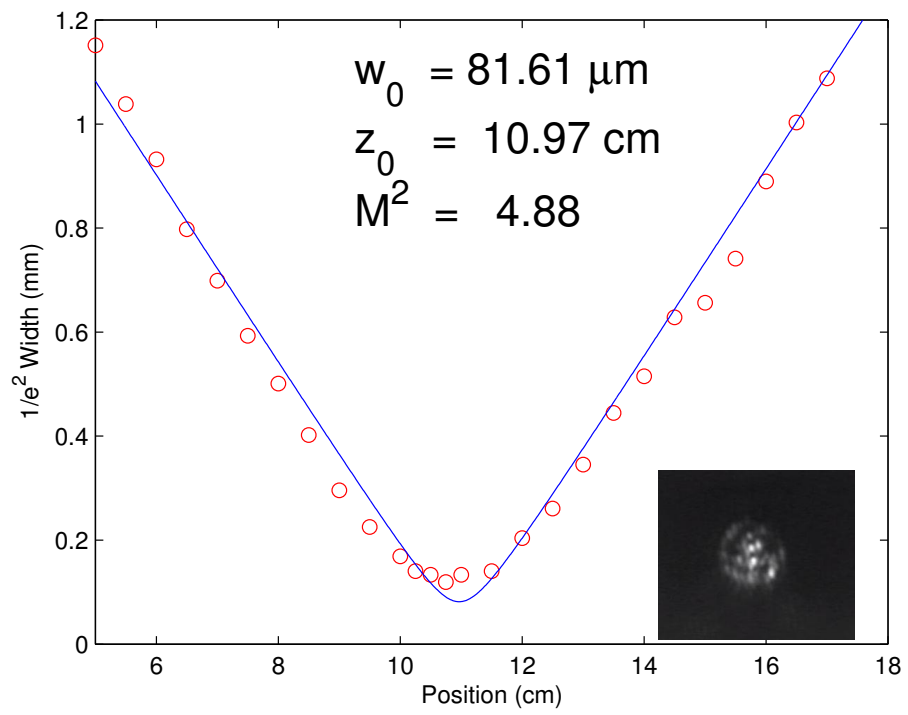

Figure 7.7: $\mathrm{M}^{2}$ measurement for the multimode waveguide. The $1 / \mathrm{e}^{2}$ width was plotted as a function of distance when focused using a $10 \mathrm{~cm}$ focal length lens. Subset picture shows the far field output mode profile in a $250 \mu m \times 250 \mu m$ image.

The $\mathrm{M}^{2}$ measurement was then repeated for the single mode waveguide. The measured beam widths and subsequent fit can be seen in Figure 7.8. From the fit, the single mode waveguides produced a beam with an $\mathrm{M}^{2}$ value of 1.41 . 


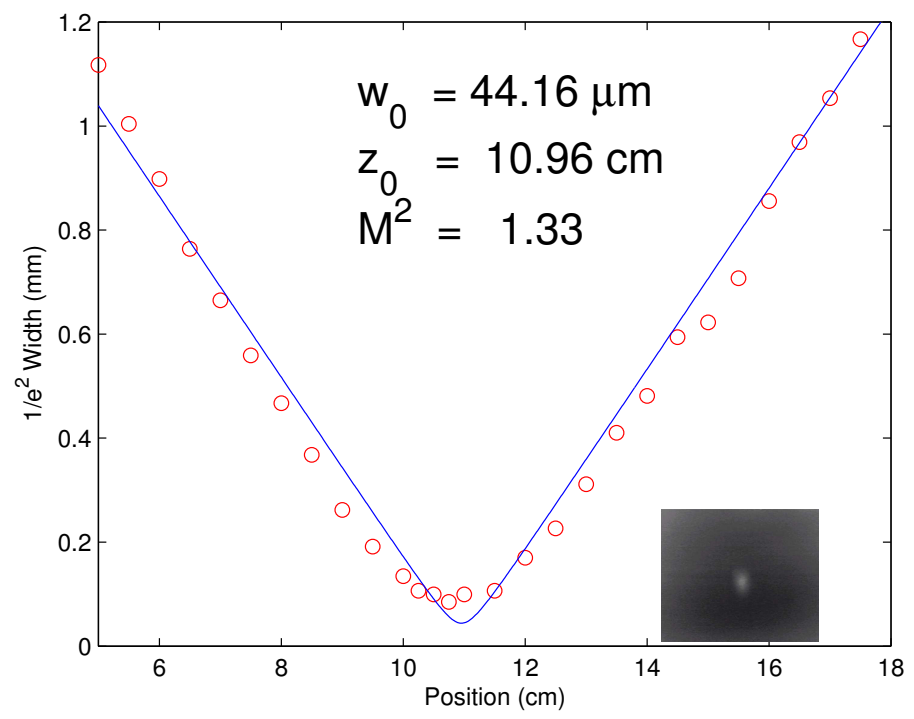

Figure 7.8: $\mathbf{M}^{2}$ measurement for the single mode waveguide. The $1 / e^{2}$ width was plotted as a function of distance when focused using a $10 \mathrm{~cm}$ focal length lens. Subset picture shows the far field output mode profile in a $120 \mu m \times 120 \mu m$ image.

\subsection{Co-Doped Waveguides}

With the goal of creating efficient holmium based Mid-IR waveguide lasers, the next step is to leverage existing fiber coupled diode sources to pump the waveguide lasers. Unfortunately, $1908 \mathrm{~nm}$ diodes are not a mature technology forcing the use of either a cascaded pumping scheme or an additional dopant for energy transfer. It has been previously shown that flashlamp pumping of $\mathrm{Yb}: \mathrm{Ho}$ :YAG is more efficient that using a thulium, or similar, sensitizer[108]. Fortunately, $\mathrm{Yb}^{3+}$ has a strong absorption at $940 \mathrm{~nm}$, which corresponds to a readily available diode wavelength. A diode pumped $2 \mu m$ laser can be realized by pumping the $\mathrm{Yb}^{3+}$ ion with a $940 \mathrm{~nm}$ diode laser, which then non-resonantly transfers to the $\mathrm{Ho}^{3+}$ ion $[109,110]$. The basic energy level diagram for this pumping configuration can be seen in Figure 7.9. The $\mathrm{Yb}^{3+}$ acts as a sensitizer for the $\mathrm{Ho}^{3+}$ ions by absorbing the $940 \mathrm{~nm}$ radiation on the ${ }^{2} \mathrm{~F}_{7 / 2} \longrightarrow{ }^{2} \mathrm{~F}_{5 / 2}$ transition. The energy then transfers to the 
$\mathrm{Ho}^{3+}$ ion via non-resonant energy transfer. Finally, through spontaneous decay from the ${ }^{5} \mathrm{I}_{6} \longrightarrow{ }^{5} \mathrm{I}_{7}$, the upper laser level of the $2 \mu m$ transition is pumped. The basic premise of this pumping scheme relies on the excited state lifetime of the ${ }^{5} \mathrm{I}_{6}$ to be much shorter than the ${ }^{5} \mathrm{I}_{7}$ as to not bottleneck the $2 \mu m$ laser transition. The relative lifetimes of these two transitions are approximately $400 \mu s$ and $7.8 \mathrm{~ms}$ respectively [111], thus bottlenecking won't occur on the laser transition. In addition, the energy exchange rate has previously been measured to be $45.3 \times 10^{3} / \mathrm{s}$ [111].

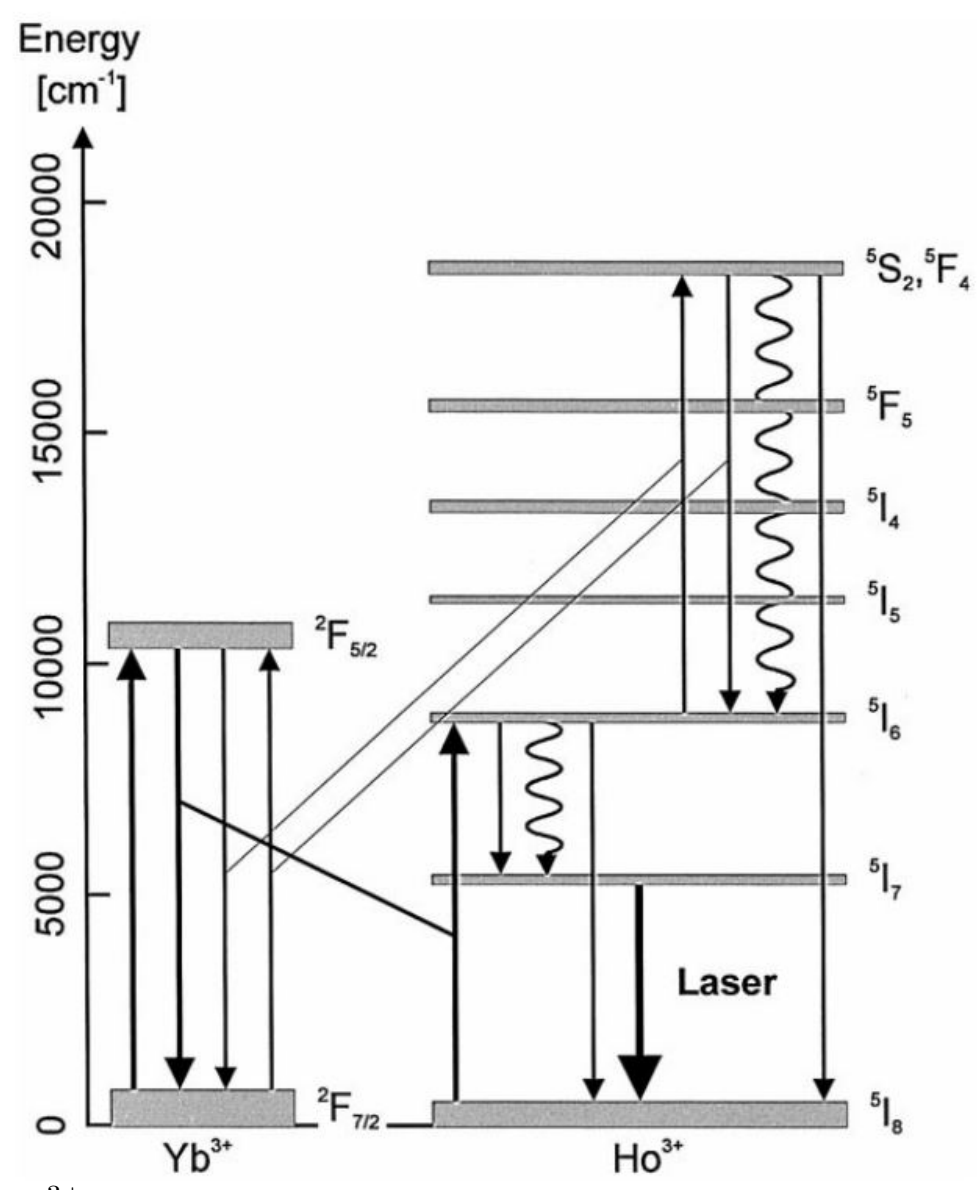

Figure 7.9: $\mathrm{Yb}^{3+} / \mathrm{Ho}^{3+}$ energy level diagram illustrating the pumping scheme of the co-doped material. The diagram has been reproduced from Rothacher et al. [109].

To this end, a sample of Yb:Ho:YAG was doped with 2at.\% holmium and 15at.\% ytterbium and inscribed with a variety of waveguides for testing. The parameters used for inscribing waveguides 
into this sample were identical to those used for the multimode waveguides. However, due to the presence of $\mathrm{Yb}^{3+}$ ions, the sample was prone to cracking during inscription due to linear absorption of the $1045 \mathrm{~nm}$ inscription laser. Note that this IMRA inscription laser is a Yb:fiber laser. Figure 7.10 represents the best waveguides inscribed in the co-doped sample. The crack formation propagates out from the laser induced damage region, thus allowing light propagation in the waveguide core. Many of the waveguides inscribed in the co-doped sample formed cracks propagating inward from the region modified by the laser. The presence of cracking in the core of the waveguide significantly increased losses and prevented guiding in most of the waveguides that were tested.

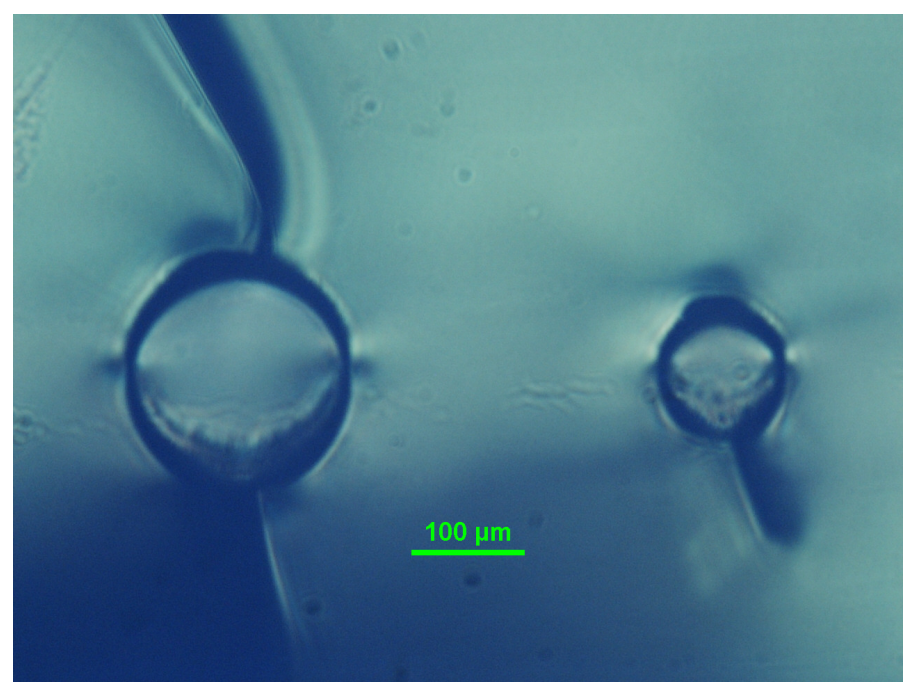

Figure 7.10: Outward radial cracking of waveguides inscribed in the Yb:Ho:YAG sample.

To initially test the viability of the co-doped waveguides, we first in-band pumped the sample with a thulium fiber laser operating at $1908 \mathrm{~nm}$ using the setup shown in Figure 7.3. The optimum performance obtained from the $80 \mu m$ waveguide can be seen in Figure 7.11. The waveguide had a maximum output power of $325 \mathrm{~mW}$ with a slope efficiency of $9 \%$ using a $70 \%$ reflective outcoupler. The spectral output of the waveguide was also measured and closely followed that of 
the multimode waveguides. The $50 \%$ and $70 \%$ reflective outcouplers had an operating wavelength of $2090 \mathrm{~nm}$. The $90 \%$ reflective outcoupler shifted the operating wavelength to $2120 \mathrm{~nm}$. The laser performance of the co-doped sample is highly decreased from the unitary doped sample. The sample had a measured loss of $1.4 \mathrm{~dB} / \mathrm{cm}$, which is approximately the same loss as the unitary doped sample.

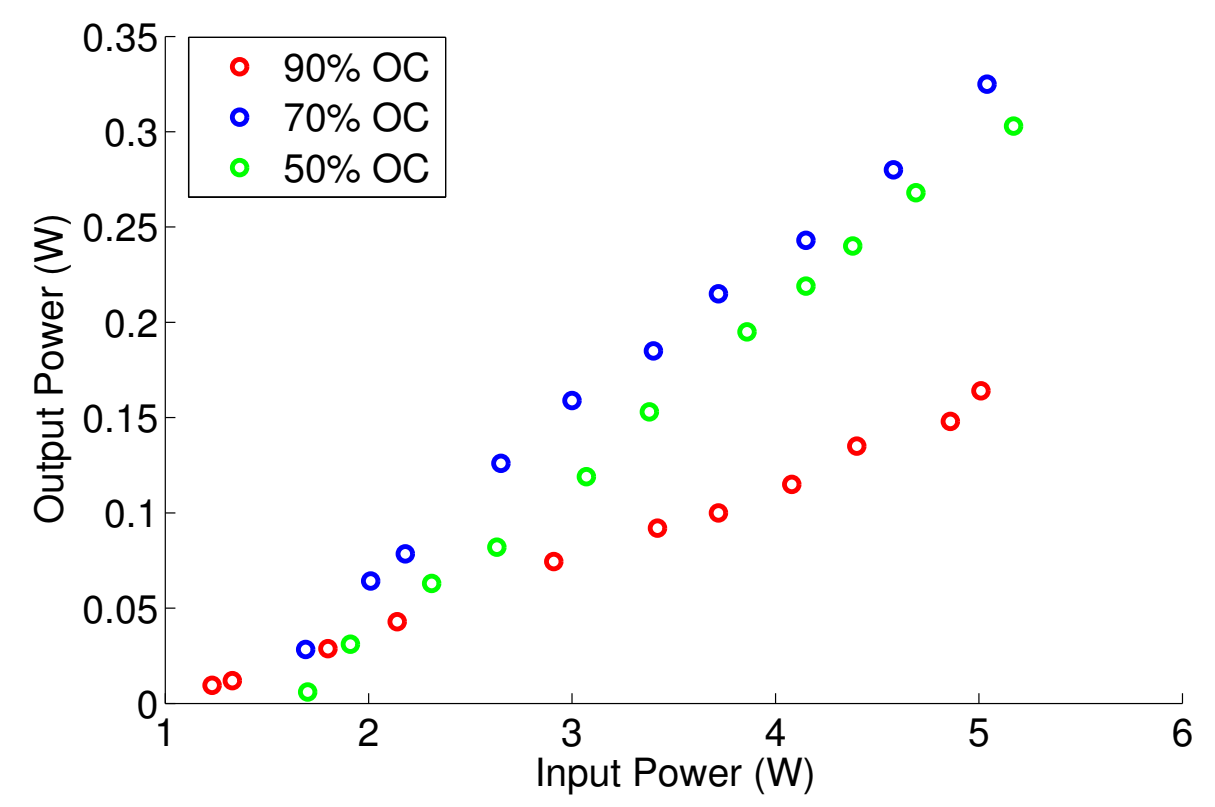

Figure 7.11: Output power for in-band pumping of the Yb:Ho:YAG waveguide.

Transitioning to diode pumping of the Yb:Ho:YAG sample required changing many of the cavity optics described in Section 7.3. However, the basic overall geometry remained the same as seen in Figure 7.12. In Figure 7.12, M1 was a $45 \mathrm{deg}$ incoupler that was AR coated for $940 \mathrm{~nm}$ and HR for $2 \mu \mathrm{m}, \mathrm{M} 2$ was a variable precentage outcoupler, M3 was an HR mirror for $2 \mu \mathrm{m}$ and L1\&L2 were uncoated $\mathrm{CaF}_{2}$ lens with a focal length of $5 \mathrm{~cm}$. The co-doped sample was mounted on a water cooled heatsink and kept at a temperature of $15^{\circ} \mathrm{C}$. 


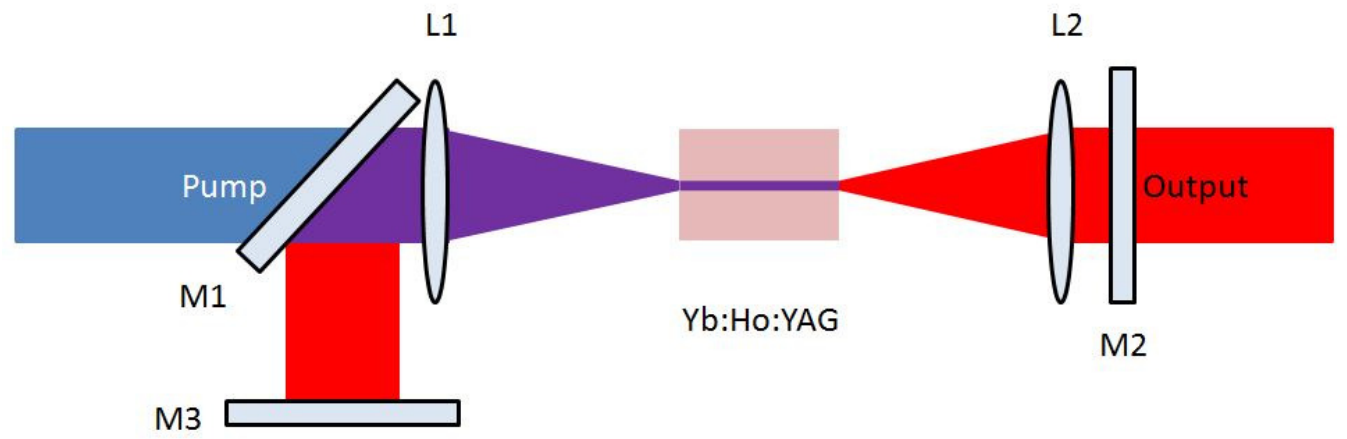

Figure 7.12: Cavity configuration used for $940 \mathrm{~nm}$ pumping of the Yb:Ho:YAG waveguide sample.

Utilizing a DILAS $40 \mathrm{~W}, 940 \mathrm{~nm}$ pump diode and a $200 \mu \mathrm{m}$ diameter, 0.22 NA multimode fiber, several attempts were made to produce output from the co-doped sample. However, no lasing action was observed. A large amount of upconversion was observed from the sample, Figure 7.13. While sample exhibited strong $2 \mu m$ florescence and exhibited excellent guiding properties, the crystal cracked under the stress of the thermal load, destroying all available waveguides. Future research will focus on optimization of the ULI parameters to minimize the stress inside of the codoped material. In addition, thermal annealing of the sample will be investigated as a technique to decrease crystal stress. 


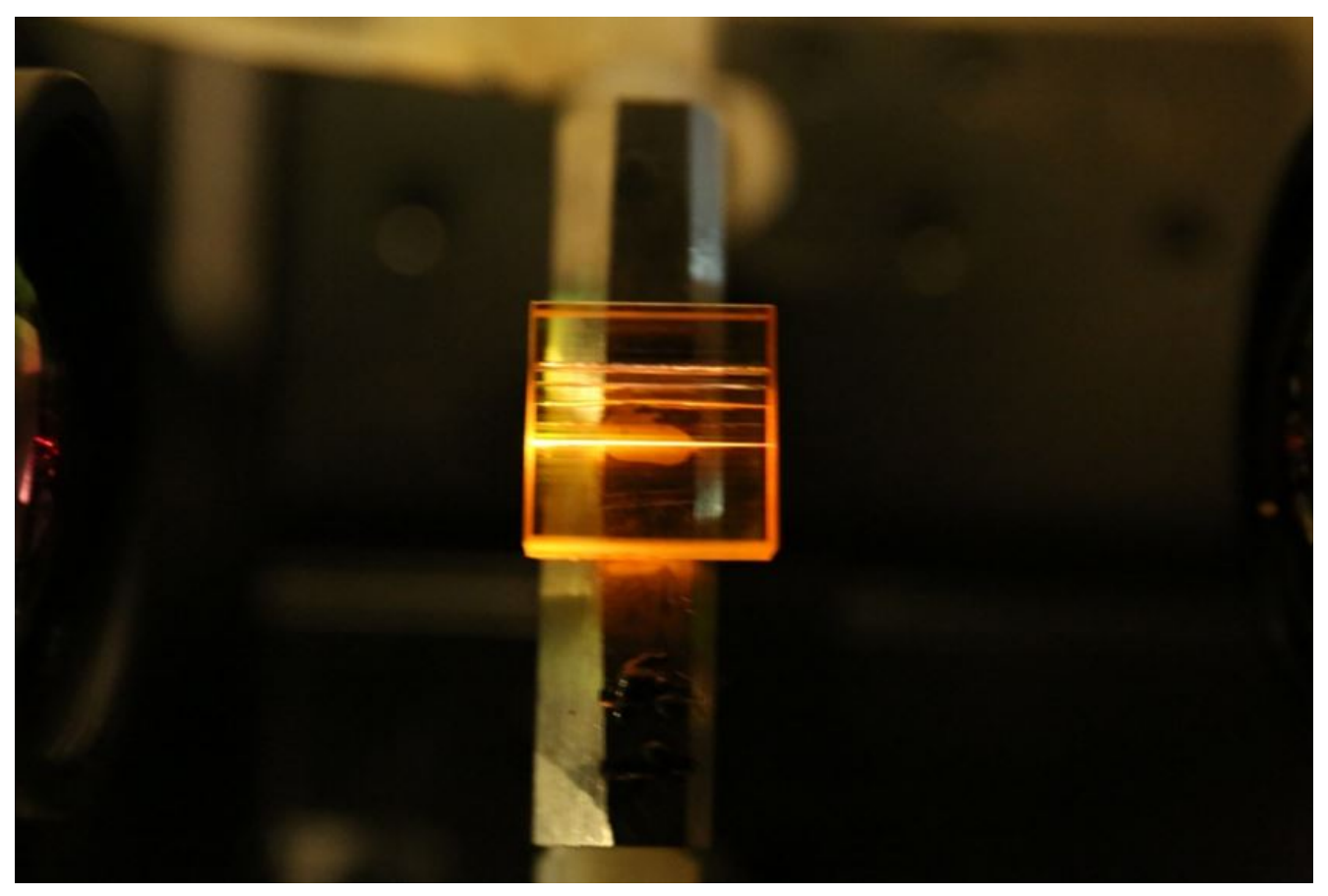

Figure 7.13: Upconversion in the Yb:Ho:YAG sample pumped by $940 \mathrm{~nm}$ diodes.

\subsection{Conclusion}

In conclusion, single and multimode waveguides in Ho:YAG have been demonstrated using two different inscription recipes. The single mode waveguides were created in a 0.5 at. $\%$ doped sample with a core diameter of $50 \mu \mathrm{m}$. Single mode operation of the waveguide laser produced a maximum output of $1.77 \mathrm{~W}$ with a slope efficiency of $16 \%$. The multimode waveguides were also inscribed in a $0.5 \mathrm{at} . \%$ doped sample, but had a larger core diameter of $80 \mu \mathrm{m}$. The multimode waveguides produced a maximum output power of $1.89 \mathrm{~W}$ with a slope efficiency of $29 \%$. Additionally, a co-doped sample of Ho:Yb:YAG was inscribed with waveguides. The waveguides produced lasing action at $2 \mu m$ using in-band pumping, albeit with decreased efficiency compared to the single and multimode waveguides. Direct diode pumping of the sample at $940 \mathrm{~nm}$ showed strong guiding and florescence at $2 \mu \mathrm{m}$, but no lasing was observed. 


\section{CHAPTER VIII}

\section{MODELING}

For solid state lasers, many authors have shown the validity of modeling transisition metals as 4-level laser systems [82]. The main assumption with modeling transition metal lasers, such as Cr:ZnSe or Fe:ZnSe, as a 4-level laser system is due to the fast vibronic relaxation of the crystal field split energy levels. The fast vibronic relaxation allows the treatment of the total number of excited ions in the upper laser level and lower laser level to be summed as the total laser population, thus allowing numerical modeling of these materials as a 2-level system. This assumption is valid only if the intraband transition lifetime is much faster than the upper-state lifetime of the laser source. In addition, due to the waveguide nature of the device, we do not have to account for the change in the beam profile as the beam propagates through the gain medium, thus there is no intensity change due to focusing. We can now set forth a basic configuration for modeling these laser devices. First, we can assume a 2-band picture for the rate equations, Figure 8.1.

In Figure 8.1, we denote the absorption and emission cross sections as $\sigma_{01}$ and $\sigma_{10}$, populations as $n_{0}$ and $n_{1}$ and the excited state lifetime as $\tau_{10}$. We can utilize this picture to formulate a set of differential equations to model the laser action of the device. The change in the population of level $N_{e}$ and $N_{g}$ as a function of time can be written as:

$$
\begin{gathered}
\frac{d n_{1}}{d t}=\sigma_{01}(P) \frac{I_{P}}{h \nu_{P}} n_{0}+\sigma_{01}(L) \frac{I_{L}}{h \nu_{L}} n_{0}-\sigma_{10}(P) \frac{I_{P}}{h \nu_{P}} n_{1}-\sigma_{10}(L) \frac{I_{L}}{h \nu_{L}} n_{1}-\frac{1}{\tau_{10}}, \\
\frac{d n_{0}}{d t}=-\sigma_{01}(P) \frac{I_{P}}{h \nu_{P}} n_{0}-\sigma_{01}(L) \frac{I_{L}}{h \nu_{L}} n_{0}+\sigma_{10}(P) \frac{I_{P}}{h \nu_{P}} n_{1}+\sigma_{10}(L) \frac{I_{L}}{h \nu_{L}} n_{1}+\frac{1}{\tau_{10}} .
\end{gathered}
$$




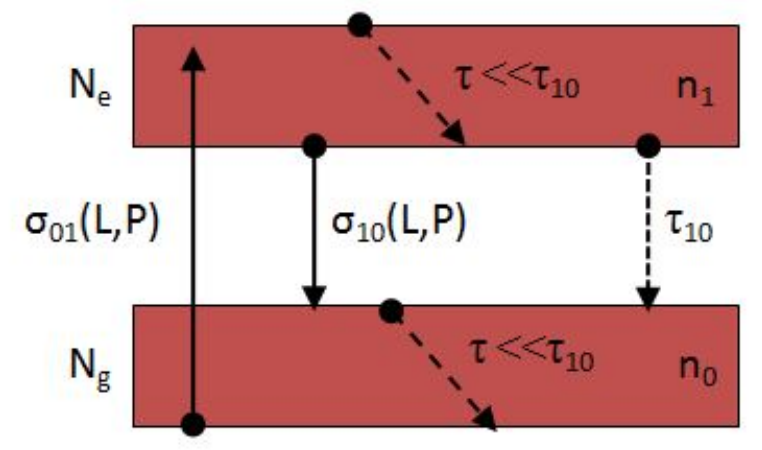

Figure 8.1: Basic picture of a 2-level laser band structure.

Noting that under CW pumping, the rate of change of the excited state $\left(N_{e}\right)$ will equal zero $\left(\frac{d n_{1}}{d t}=0\right)$, thus we can separate Equation 8.1 such that $n_{0}$ and $n_{1}$ are separable:

$$
n_{0}\left(\sigma_{01}(P) \frac{I_{P}}{h \nu_{P}}+\sigma_{01}(L) \frac{I_{L}}{h \nu_{L}}+\frac{1}{\tau_{10}}\right)=n_{1}\left(\sigma_{10}(P) \frac{I_{P}}{h \nu_{P}}+\sigma_{10}(L) \frac{I_{L}}{h \nu_{L}}-\frac{1}{\tau_{10}}\right) .
$$

Rearranging the solution, the ground state population $\left(n_{0}\right)$ can be formulated as a function of $n_{1}:$

$$
n_{0}=\frac{n_{1}\left(\sigma_{10}(P) \frac{I_{P}}{h \nu_{P}}+\sigma_{10}(L) \frac{I_{L}}{h \nu_{L}}-\frac{1}{\tau_{10}}\right)}{\left(\sigma_{01}(P) \frac{I_{P}}{h \nu_{P}}+\sigma_{01}(L) \frac{I_{L}}{h \nu_{L}}+\frac{1}{\tau_{10}}\right)} .
$$

Noting that the total population is the sum of the ground state $\left(n_{0}\right)$ and excited state populations $\left(n_{1}\right)$, the solution can be formulated in terms of the total number of ions:

$$
n_{0}=\frac{n_{T}\left(\sigma_{10}(P) \frac{I_{P}}{h \nu_{P}}+\sigma_{10}(L) \frac{I_{L}}{h \nu_{L}}-\frac{1}{\tau_{10}}\right)}{\left(\sigma_{01}(P) \frac{I_{P}}{h \nu_{P}}+\sigma_{01}(L) \frac{I_{L}}{h \nu_{L}}+\frac{1}{\tau_{10}}+\sigma_{10}(P) \frac{I_{P}}{h \nu_{P}}+\sigma_{10}(L) \frac{I_{L}}{h \nu_{L}}\right)} .
$$

With the intensity dependent populations formulated, we need to define the differential equations in terms of distance. This is easily done by noting that the distance dependent intensity is 
related to the intensity at that given point in the gain medium, the cross section and the current number of carriers in the laser levels:

$$
\begin{gathered}
\frac{d I_{P}}{d z}=-I_{P}\left(-\sigma_{01}(P) n_{0}-\sigma_{10}(P) n_{1}\right) \\
\frac{d I_{L}}{d z}=I_{L}\left(\sigma_{10}(L) n_{1}-\sigma_{01}(L) n_{0}\right) .
\end{gathered}
$$

The equations shown in Equation 8.5 coupled with Equation 8.4 will allow the implementation of an ordinary differential equation solver to produce a solution for arbitrary lengths of gain media. If we assume the parameters listed in Table 8.1, a basic test of the model can be conducted in a single pass amplifier configuration.

Table 8.1: General parameters for testing the differential equations shown in Equation 8.5.

\begin{tabular}{|c|c|c|c|c|}
\hline Pump Power $(\mathrm{W})$ & Signal Power $(\mathrm{W})$ & Pump Radius $(\mu \mathrm{m})$ & Signal Radius $(\mu \mathrm{m})$ & Gain Length $(\mathrm{cm})$ \\
\hline 1 & 0.2 & $60 \mu \mathrm{m}$ & $60 \mu \mathrm{m}$ & 1 \\
\hline
\end{tabular}

The single pass amplifier result can be seen in Figure 8.2. The model predicts a maximum output power of $0.78 \mathrm{~W}$ with an extraction efficiency of approximately $60 \%$. These results are typical of this material and other researchers have shown similar results [82]. 


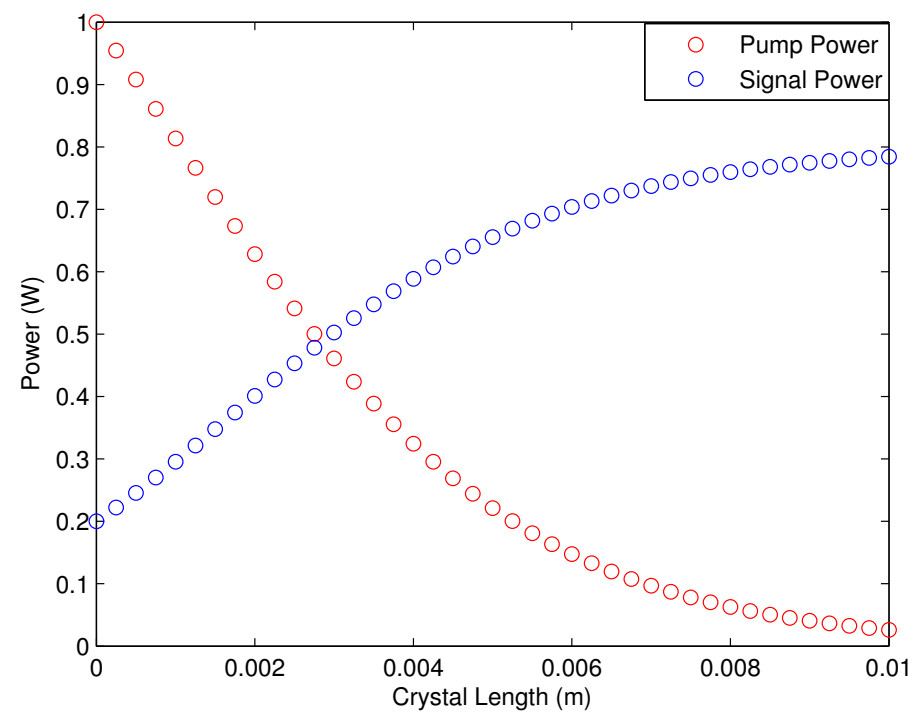

Figure 8.2: Modeled output from a single pass amplifier using the rate equations set forth in Equation 8.5. The model predicted a maximum output of $0.78 \mathrm{~W}$ through a $1 \mathrm{~cm}$ amplifier with $200 \mathrm{~mW}$ of seed power and $1 \mathrm{~W}$ of pump power.

Now, the model can be applied to a resonator. The physics behind the rate equations is identical, however the solution is now iterated over several crystal propagation distances. The advantage of the waveguide configuration is that the pump and signal beam diameters can be treated as constant across the entire sample lengths, thus focusing can be neglected. In order to simulate actual resonator dynamics, a signal power of $1 \times 10^{-35} W$ was used. After solving one cavity length, the signal is multiplied by the outcoupler reflectivity then propagated though the cavity twice to represent a round-trip. The output power is then compared to the power of the previous iteration. If the gain is greater than unity, representing the small signal gain, then the solution keeps iterating until a gain of unity is reached. Unity gain represents a steady-state laser oscillation such that the gain per round trip matches the gain of the cavity. Figure 8.3 shows the round-trip gain of the system as a function of round-trip iterations. After 23 iterations, the round-trip gain of the cavity has dropped to from the small signal gain of approximately 200 to a round-trip gain of 1. 


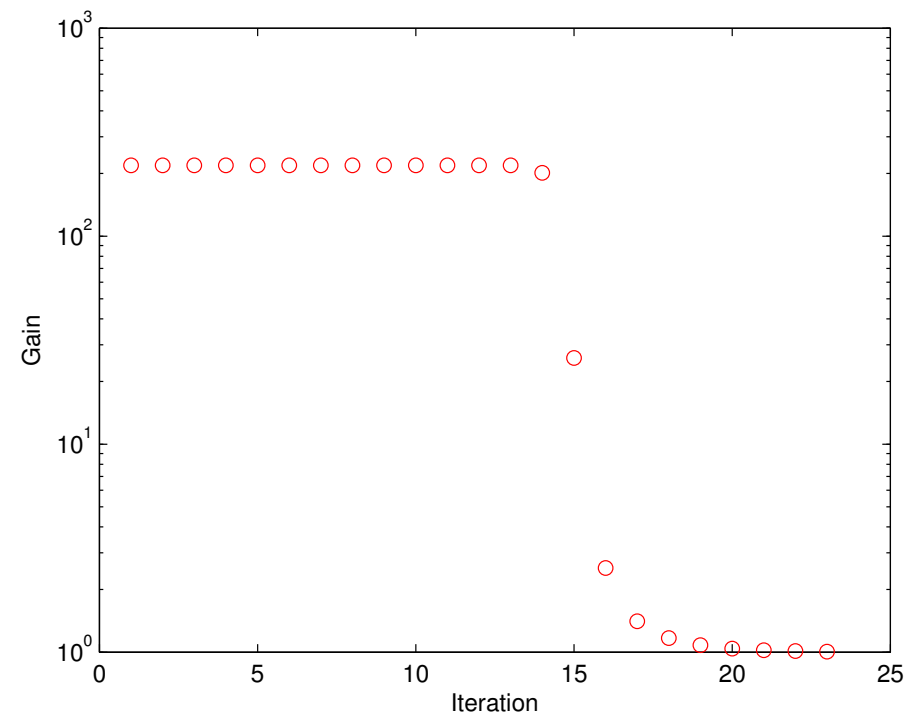

Figure 8.3: Round-trip gain of a simulated waveguide laser based on Cr:ZnSe. The iteration number represents the number of cavity round-trips.

This method can now be applied to several different input powers, thus the efficiency for the laser can be extracted from the model, Figure 8.4. The model predicts a maximum output of $7.7 \mathrm{~W}$, which is the quantum limit for $1.9 \mu \mathrm{m}$ pumping of $\mathrm{Cr}: \mathrm{ZnSe}$ emitting at $2.45 \mu \mathrm{m}$. 


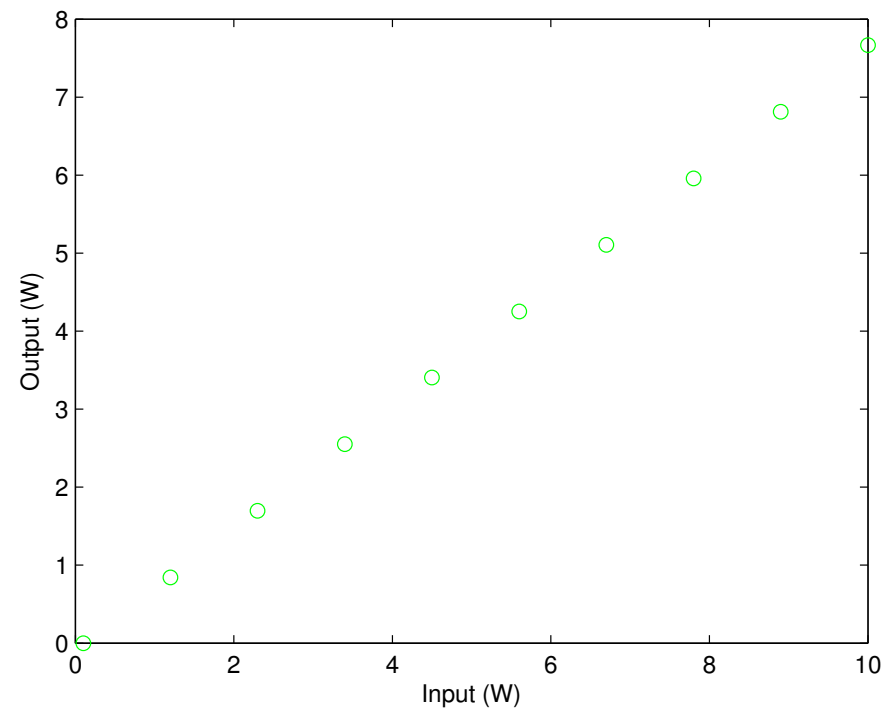

Figure 8.4: Modeled slope efficiency for a Cr:ZnSe laser with a $70 \%$ reflective outcoupler and no loss or thermal effects and the parameters from Table 8.1.

However, this model does not include loss, other than the loss from the mirrors. In order to incorporate losses, an additional term needs to be subtracted from the rate equations, Equation 8.6.

$$
\begin{gathered}
\frac{d I_{P}}{d z}=-I_{P}\left(\sigma_{01}(P) n_{0}-\sigma_{10}(P) n_{1}\right)-\gamma_{P} \\
\frac{d I_{L}}{d z}=I_{L}\left(\sigma_{10}(L) n_{1}-\sigma_{01}(L) n_{0}\right)-\gamma_{L}
\end{gathered}
$$

The terms $\gamma_{P}$ and $\gamma_{L}$ are the loss per unit length of the waveguide, which has been measured in terms of propagation loss, $d B / \mathrm{cm}$. The typical value for propagation loss for active ULI waveguide lasers is approximately $1 \mathrm{~dB} / \mathrm{cm}$, which corresponds to a loss of $20 \% / \mathrm{cm}$. Note that the loss values are just a constant offset to the solution of the differential equations, which affects both the laser threshold and the slope efficiency. Noting the approximate loss for ULI waveguides, a table of modeled output values can be assembled. Table 8.2 shows the tabulated results for a $40 \mu \mathrm{m}$ diameter waveguide assuming no loss, no thermal effects and $10 \mathrm{~W}$ of pump power. 
Table 8.2: Modeled output power from ULI waveguides.

\begin{tabular}{|c|c|c|c|}
\hline Material & Input Power & Output Power & Slope Efficiency \\
\hline Cr:ZnSe & $10 W$ & $7.7 W$ & $77 \%$ \\
\hline Fe:ZnSe & $10 W$ & $6.9 W$ & $69 \%$ \\
\hline Ho:YAG & $10 W$ & $9.1 W$ & $91 \%$ \\
\hline
\end{tabular}

The values in Table 8.2 follow exactly with theory. The same model can now be utilized to determine the output with a loss value of $1 \mathrm{~dB} / \mathrm{cm}$ and reflectivities from $20 \%$ to $90 \%$. The values obtained from the model match closely from the optimal outcoupler reflectivities found in literature $[84,112,113]$

Table 8.3: Output for various outcoupler reflectivities for Cr:ZnSe, Fe:ZnSe and Ho:YAG materials. Experimentally obtained output powers and slope efficiencies are included for comparison.

\begin{tabular}{|c|c|c|c|c|c|}
\hline Material & $\% \mathrm{R}$ & Output Power & Modeled Slope & Exp. Output Power & Exp. Slope \\
\hline Cr:ZnSe & $20 \%$ & $N / A$ & $N / A$ & $N / A$ & $N / A$ \\
\hline & $50 \%$ & $4.78 W$ & $35 \%$ & $1.1 W[30]$ & $21 \%[30]$ \\
\hline & $70 \%$ & $5.01 W$ & $58 \%$ & $5.2 W$ & $41 \%$ \\
\hline & $90 \%$ & $2.10 W$ & $39 \%$ & $0.3 W[30]$ & $9 \%[30]$ \\
\hline Fe:ZnSe & $18 \%$ & $1.20 W$ & $35 \%$ & $60 \mathrm{~mW}[31]$ & $15 \%[31]$ \\
\hline & $40 \%$ & $5.35 W$ & $65 \%$ & $1 W$ & $58 \%$ \\
\hline & $80 \%$ & $3.86 W$ & $45 \%$ & $80 \mathrm{~mW}[31]$ & $11 \%[31]$ \\
\hline & $90 \%$ & $1.95 W$ & $37 \%$ & $40 \mathrm{~mW}[31]$ & $7 \%[31]$ \\
\hline Ho:YAG & $20 \%$ & $N / A$ & $N / A$ & $N / A$ & $N / A$ \\
\hline & $50 \%$ & $4.24 W$ & $46 \%$ & $1.88 W$ & $29 \%$ \\
\hline & $70 \%$ & $3.66 W$ & $34 \%$ & $1.88 W$ & $27 \%$ \\
\hline & $90 \%$ & $1.91 W$ & $23 \%$ & $0.91 W$ & $11 \%$ \\
\hline
\end{tabular}

From Table 8.3, it can be seen that the experimental values obtained in this work closely match the theoretical values calculated using the rate equation model. For comparison where experimental data was not available, values from other authors were used for comparison purposes. Value from other authors differ dramatically from the modeled values, which can be explained by higher 
propagation losses, non-optimal coupling, or unoptimized laser parameters. This can be used in conjunction with the next section to determine the performance of the waveguide lasers.

\subsection{Theoretical Limit}

Lasing operation is expected to reduce the heat load but at a certain point of pumping the situation will become unstable. A small increase in temperature will lead to an increased rate of non-radiative relaxation which in turn leads to decreased lasing and greater temperature rise. This feedback can continue until lasing stops completely. An approximate calculation of the abovethreshold pump power at which lasing ceases can be done by recalling:

$$
P_{\text {out }}=S\left(P_{\text {in }}-P_{\text {th }}\right)
$$

where $P_{\text {out }}$ is the laser output power, $P_{\text {in }}$ is the laser pump power, $P_{t h}$ is the laser threshold power and $S$ is the slope efficiency. For a four-level laser,

$$
P_{t h}=\kappa\left(P_{\text {sat }}\right)
$$

where $P_{\text {sat }}$ is the saturation power dependent only on the physics of the transition metal ions or rare earth ions, $A$ is the area of the pump beam and $\kappa$ is a proportionality constant that depends upon details of a specific laser resonator setup. The saturation power in our case depends upon temperature since emission lifetime depends on temperature, i.e.,

$$
P_{\text {sat }}(T)=\frac{A h \nu_{e}}{\left(\sigma_{e}+\sigma_{a}\right) \tau(T)}
$$

Where $h$ is Plancks constant, $\nu_{e}$ is the frequency of the emitted photon, $\sigma_{e}$ is the emission cross section and $\tau(T)$ is the emission lifetime. Note that for transition metal ions, as we increase pump power we expect to rapidly decrease as the temperature goes above $300 \mathrm{~K}$. That means that $I_{\text {sat }}$ and $P_{t h}$ increase. Eventually output power will no longer increase with increasing pump power at a 
critical pump power so that

$$
0=\frac{d P_{\text {out }}}{d P_{\text {in }}}=S\left(1-\frac{d P_{\text {th }}}{d P_{\text {in }}}\right)
$$

where the right side of Figure 8.9 comes from differentiating Equation 8.7 with respect to input power. Solving for $\frac{d P_{t h}}{d P_{\text {in }}}$ and using:

$$
\frac{d P_{t h}}{d P_{\text {in }}}=\kappa \frac{d P_{\text {sat }}}{d T} \frac{d T}{d P_{\text {in }}}
$$

an equation, which is dependent on measurable experimental conditions can be formulated:

$$
\frac{\kappa A h \nu_{e}}{\sigma_{e}} \frac{d(1 / \tau)}{d T} \frac{d T}{d P_{\text {in }}}=1
$$

Measured lifetimes from Deloach et al. [4] were fit to an 8th order polynomial for convenience in order to calculate the temperature derivative of $\frac{1}{\tau}$.

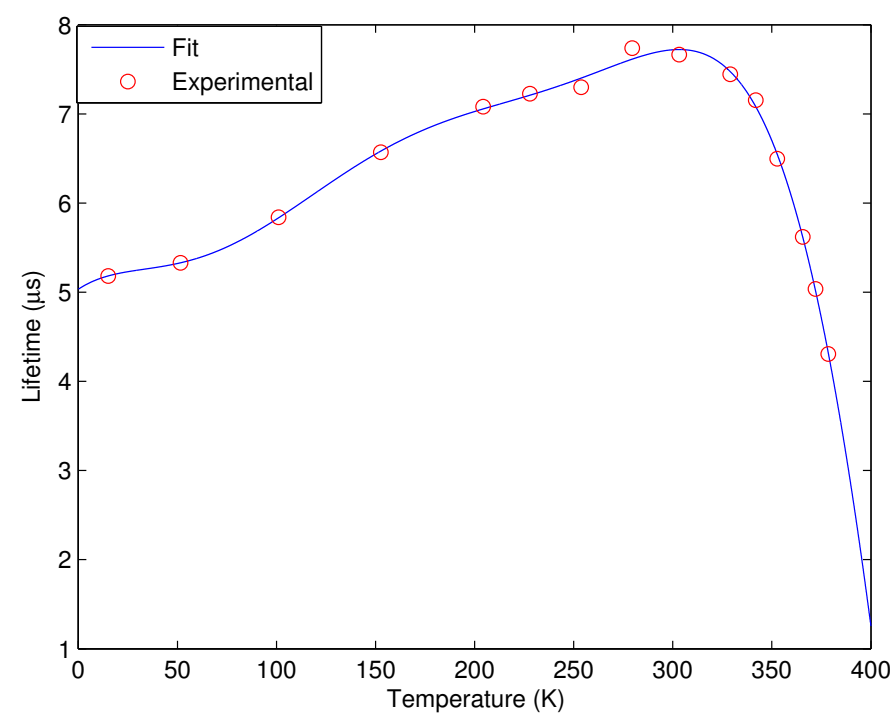

Figure 8.5: 8th order polynomial fit to the experimental data of Deloach et al. [4].

With a theoretical basis formed for quantifying the performance of waveguide devices, a COMSOL model was created to model the temperature rise inside of the waveguide. The model was 
based on the steady state thermal profile induced by CW pumping of the waveguide sample. Several simulation geometries were utilized in order to determine the laser performance as a function of waveguide depth. These geometries are shown in Figure 8.6.

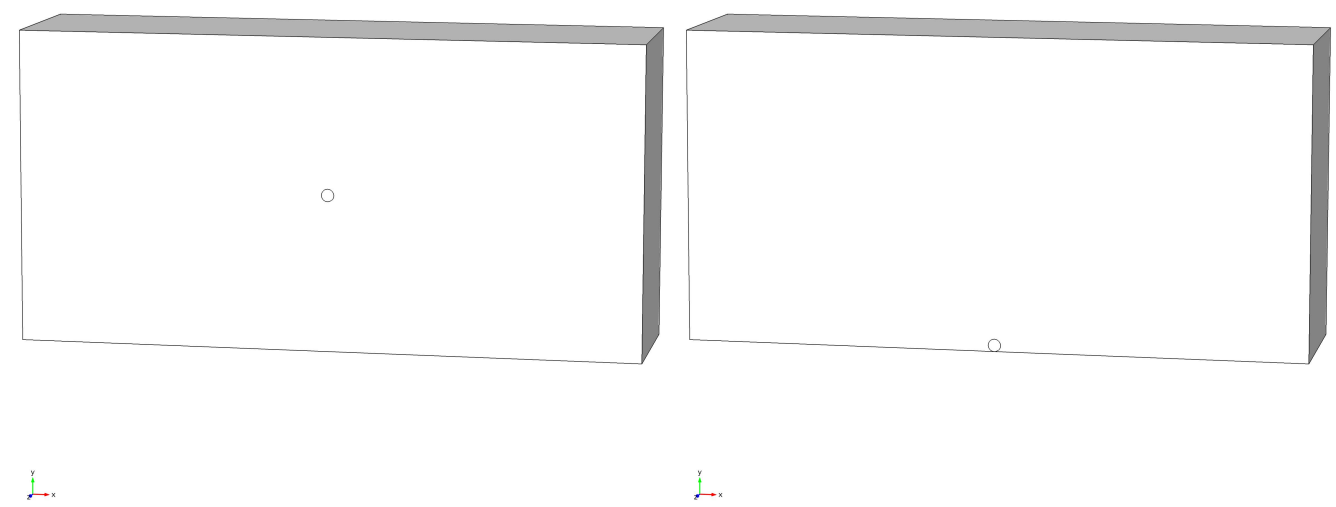
(a) Geometry 1
(b) Geometry2

Figure 8.6: (a) Geometry of a $40 \mu \mathrm{m}$ diameter waveguide structure $500 \mu \mathrm{m}$ away from the cooling surface at $y=0$. (b) Geometry of a $40 \mu \mathrm{m}$ diameter waveguide structure centered $20 \mu \mathrm{m}$ away from the cooling surface at $y=0$.

The geometries shown in Figure 8.6 represent the the extremes of the parametric sweep for waveguide depth. The simulation was run for waveguide depths varying from $500 \mu \mathrm{m}$ from the cooling surface to $20 \mu \mathrm{m}$ to the cooling face. Figure 8.10 shows the simulation results for several different pump powers, ranging from $1 W$ to $10 W$. The thermal load on the waveguide was calculated from the absorption of the gaussian beam incident on the waveguide. The thermal load due to a gaussian beam can be found by

$$
P_{d}(x, y)=\frac{2 \alpha P}{\pi r^{2}} e^{\left(\frac{-2\left(x^{2}+y^{2}\right)}{r^{2}}-\alpha z\right)} .
$$


Equation 8.13 allows the power deposited into the waveguide core to be modeled. The thermal model predictions are shown in Figure 8.7 and Figure 8.8.

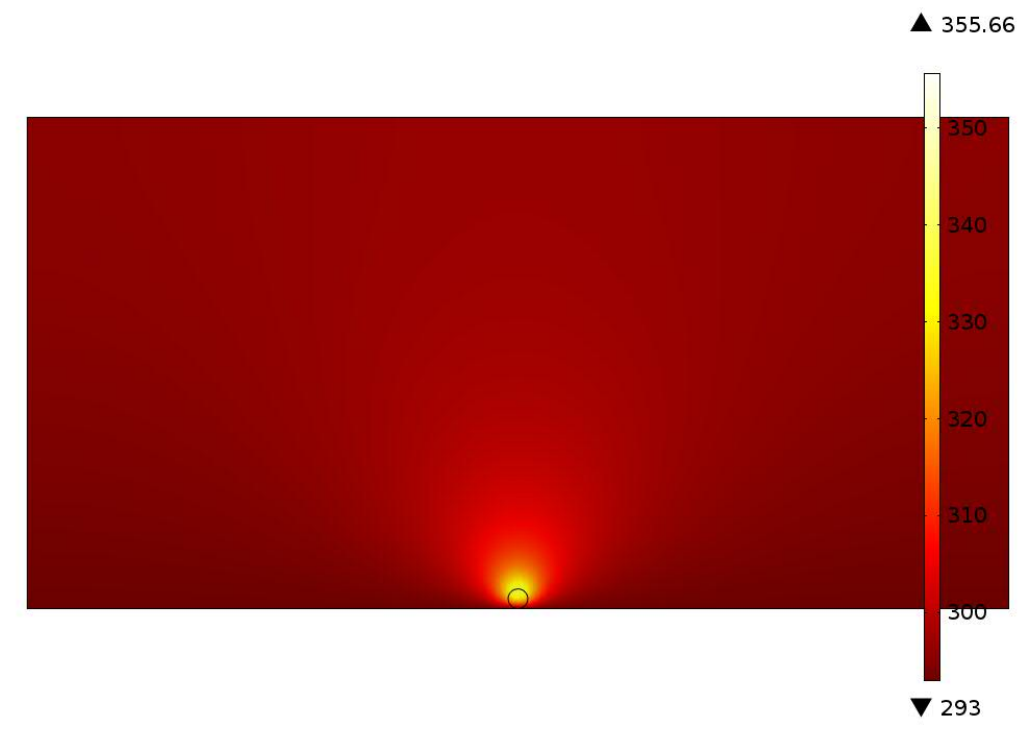

Figure 8.7: Modeled results for a $10 \mathrm{~W}$ heat source centered in a $40 \mu \mathrm{m}$ waveguide located $20 \mu \mathrm{m}$ from the cooling face. 


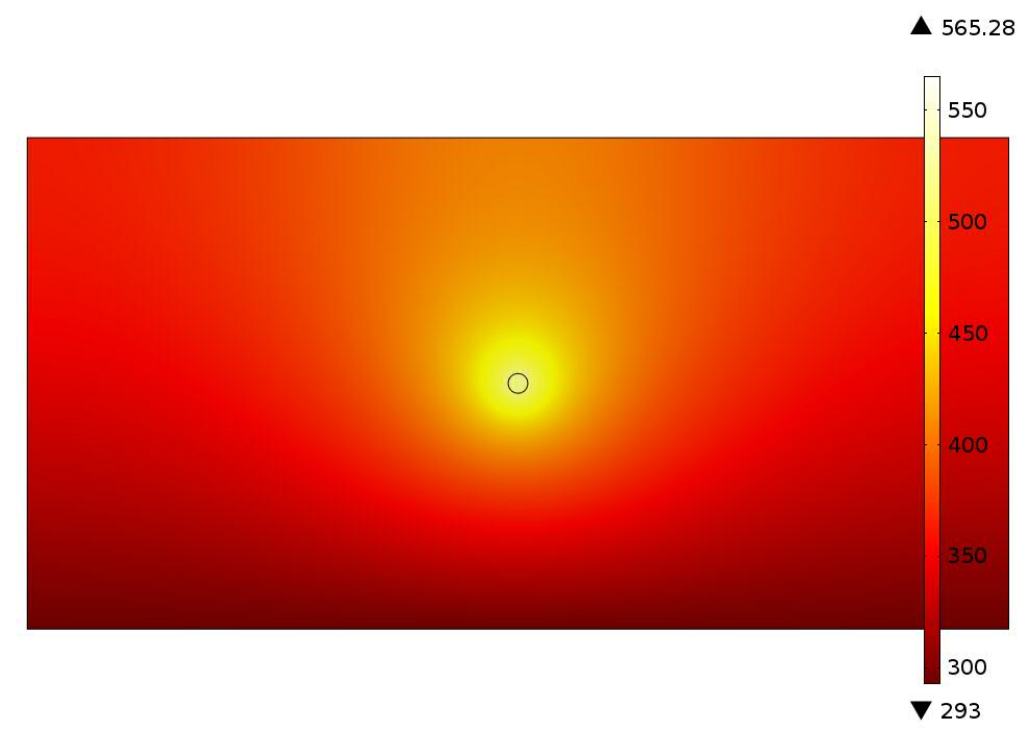

Figure 8.8: Modeled results for a $10 \mathrm{~W}$ heat source centered in a $40 \mu \mathrm{m}$ waveguide located $500 \mu \mathrm{m}$ from the cooling face.

The $\frac{d T}{d P_{\text {in }}}$ factor is the change in the core temperature as a function of input power. The COMSOL model predicts an approximate value of $\frac{d T}{d P_{i n}}=27.4 \mathrm{~K} / \mathrm{W}$ for a distance of $500 \mu \mathrm{m}$ and $\frac{d T}{d P_{\text {in }}}=6.3 \mathrm{~K} / \mathrm{W}$ for a distance of $20 \mu \mathrm{m}$. Below lasing threshold $\mathrm{dT} / \mathrm{dPin}=\mathrm{H}$. Above threshold, there is less heating since there is less chance for non-radiative relaxation; so dT/dPin $=(1-\mathrm{S}) \mathrm{H}$. 


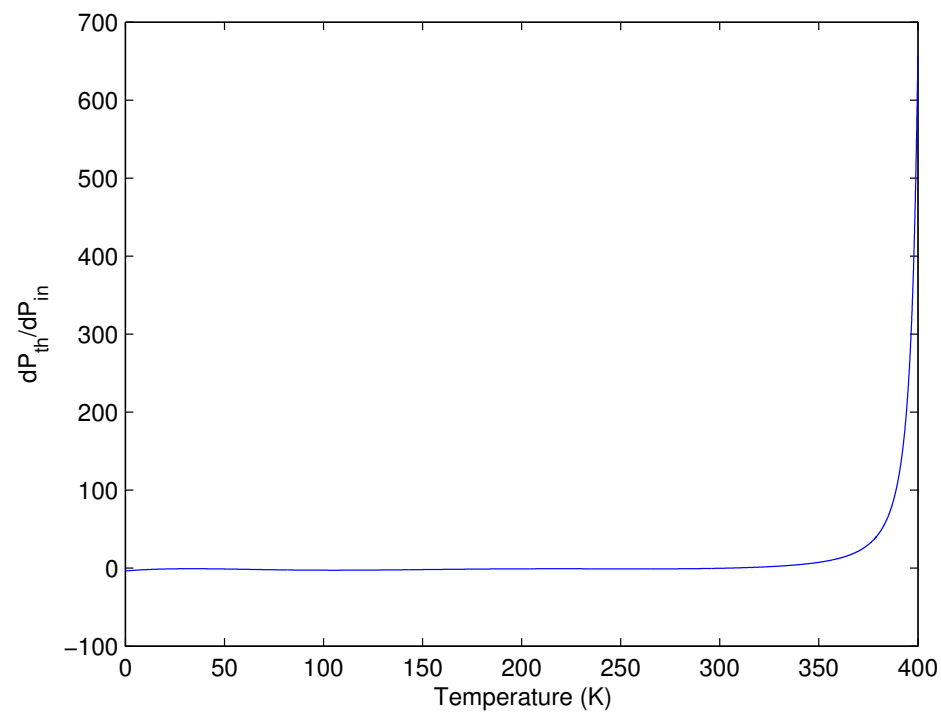

Figure 8.9: Calculated change in the threshold pump power as a function of input pump power.

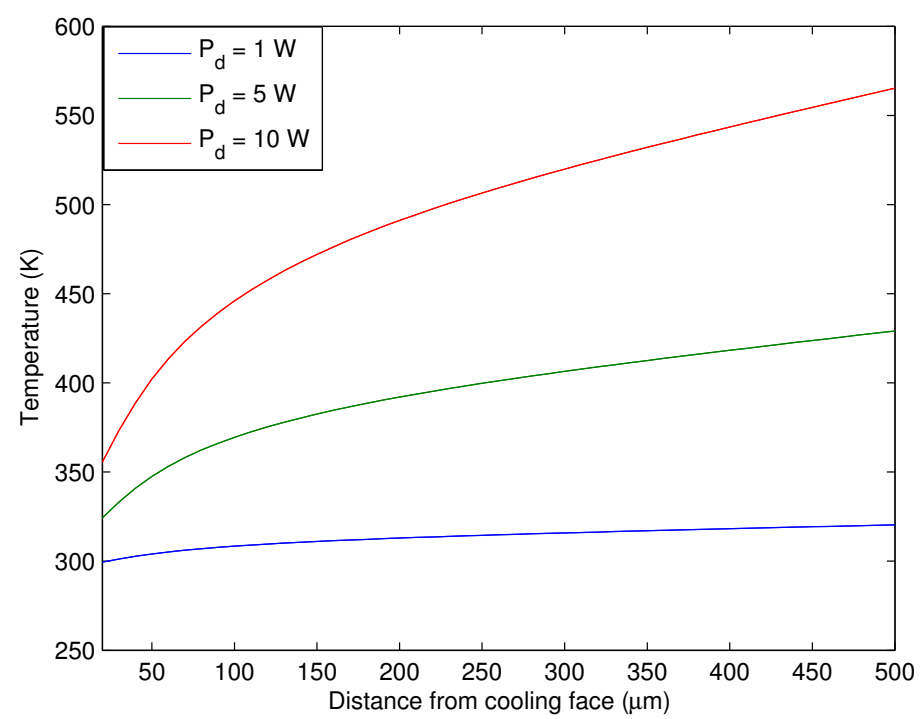

Figure 8.10: Simulated temperature as a function of waveguide depth for $\mathrm{Cr}: \mathrm{ZnSe}$. 
We have a solution of Equation 8.12 at a critical temperature of $400 \mathrm{~K}$. The critical change in temperature $\Delta T_{\text {crit }}=T_{\text {crit }}-T_{o}$ where $T_{o}$ is the starting temperature and the critical power is given in terms of input power by

$$
\Delta T_{\text {crit }}=\frac{d T}{d P_{\text {in }}}+(1-S) \frac{d T}{d P_{\text {in }}}\left(P_{\text {crit }}-P_{t h}\right)
$$

Equation 8.14 can then be solved for $P_{\text {crit }}$, yielding

$$
P_{c r i t}=\frac{\Delta T_{c r i t}-S P_{t h} \frac{d T}{d P_{i n}}}{(1-S) \frac{d T}{d P_{i n}}}
$$

Table 8.4: Summary of relevant material parameters.

\begin{tabular}{|c|c|c|c|c|}
\hline Material & $\sigma_{a}$ & $\sigma_{e}$ & $P_{t h}$ & $S$ \\
\hline Cr:ZnSe [114] & $1.1 \times 10^{-18} \mathrm{~cm}^{-2}$ & $1.2 \times 10^{-18} \mathrm{~cm}^{-2}$ & $0.1 \mathrm{~W}$ & $77 \%$ \\
\hline Fe:ZnSe [115] & $0.96 \times 10^{-18} \mathrm{~cm}^{-2}$ & $2.3 \times 10^{-18} \mathrm{~cm}^{-2}$ & $0.1 \mathrm{~W}$ & $69 \%$ \\
\hline Ho:YAG [116] & $1.2 \times 10^{-20} \mathrm{~cm}^{-2}$ & $1 \times 10^{-19} \mathrm{~cm}^{-2}$ & $0.1 \mathrm{~W}$ & $91 \%$ \\
\hline
\end{tabular}

If we assume a best case scenario for an $40 \mu \mathrm{m}$ diameter waveguide at a distance of $500 \mu \mathrm{m}$ from the cooling face of the crystal, $S=0.77$ (quantum limit for pumping with $1.9 \mu \mathrm{m}$ and lasing at $2.45 \mu \mathrm{m}), P_{t h}=0.1 \mathrm{~W}$ (best reported for Cr:ZnSe waveguides), $\Delta T_{\text {crit }}=19.7 \mathrm{~K}$ and $\frac{d T}{d P_{\text {in }}}=$ $27.4 \mathrm{~K} / \mathrm{W}$, we can calculate a critical incident pump power of approximately $2.8 \mathrm{~W}$. Given this pump power, we can expect at most $2.1 \mathrm{~W}$ of laser output from the waveguide. Note, that this is the critical power for the front region of the waveguide. Regions further downstream will reach critical temperature at slightly higher powers. This provides a maximum theoretical output attainable from the waveguides. For the waveguides demonstrated in [29], a maximum slope efficiency of $S=0.41$ was obtained with a threshold of $0.1 \mathrm{~W}$. With a slope efficiency of $41 \%$, the maximum incident pump power would be approximately $1.2 \mathrm{~W}$ with an expected output of approximately $0.45 \mathrm{~W}$. 
The calculated critical power is only for the front surface of the waveguide. Distances further away from the front facet will reach the critical power at higher pumping conditions, which accounts for the difference in predicted performance versus actual performance.

These numbers change quite dramatically as the waveguide is moved closer to the cooling facet of the crystal, Figure 8.11. Again, if we assume the best case scenario using the parameters previously stated, except the waveguide center was moved from $500 \mu \mathrm{m}$ away from the cooling face to $20 \mu \mathrm{m}$ away, a critical incident power of $25 \mathrm{~W}$ was obtained. In addition, moving the waveguide closer to the cooling face changes the values of $\Delta T_{\text {crit }}$ and $\frac{d T}{d P_{\text {in }}}$ to $37.9 \mathrm{~K}$ and $6.4 \mathrm{~K} / \mathrm{W}$ respectively. If the slope efficiency is then lowered to match experimentally obtained data, we can find a critical incident power of $10 \mathrm{~W}$, which approximately matches the performance of previously demonstrated waveguides.

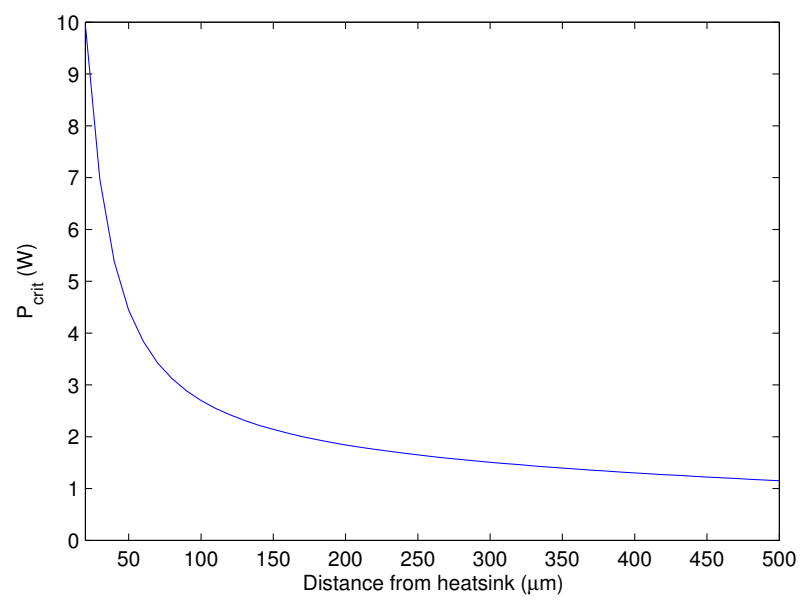

Figure 8.11: Critical pump power required for the temperature change to decrease the excited state lifetime of the laser material.

In addition, we can examine the effects of cryogenic cooling the waveguide sample. In theory, this should drop the steady-state operating temperature of the waveguide laser allowing it to reach 
higher pump powers before it thermally quenches. The same material parameters were used for these simulations. It should be noted that the emission and absorption cross-sections for this material can change as a function of temperature. Figure 8.12 and Figure 8.13 show the decreased operating temperature as compared to Figure 8.7 and Figure 8.8 .

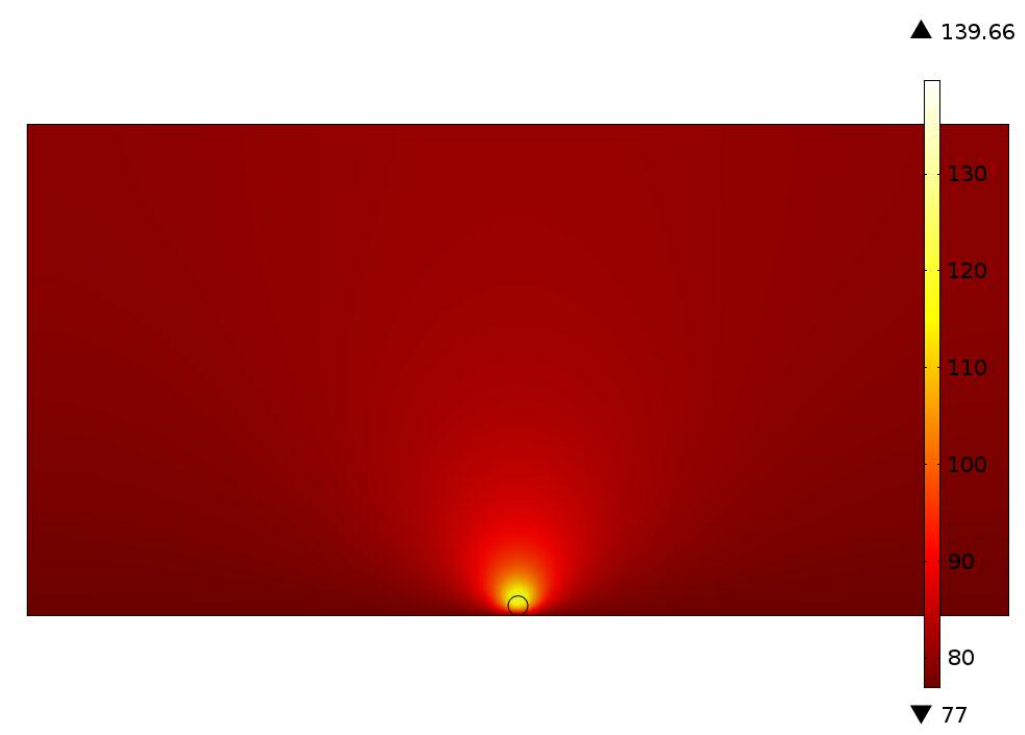

Figure 8.12: Modeled results for a $10 \mathrm{~W}$ heat source centered in a $40 \mu \mathrm{m}$ waveguide located $20 \mu \mathrm{m}$ from the cryogenically cooled face. 


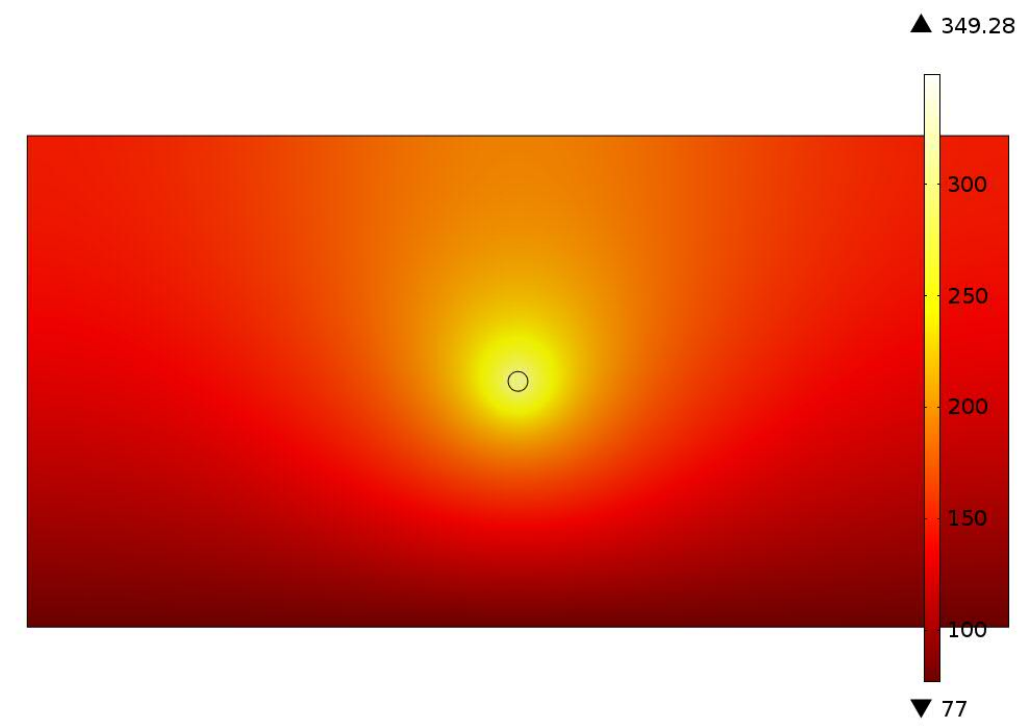

Figure 8.13: Modeled results for a $10 \mathrm{~W}$ heat source centered in a $40 \mu \mathrm{m}$ waveguide located $500 \mu \mathrm{m}$ from the cryogenically cooled face.

The effect of the decreased temperature is that the critical power level before the emission efficiency decreases is much higher, Figure 8.14. From Figure 8.14, it can be seen that under the thermal conditions produced by a $1 W$ thermal load, the critical power is never reached. For the conditions produced by $5 \mathrm{~W}$ of pump power, the critical power is reached at a depth of $100 \mu \mathrm{m}$. Finally, for $10 \mathrm{~W}$ of pump power, the critical power is achieved for all modeled depths. 


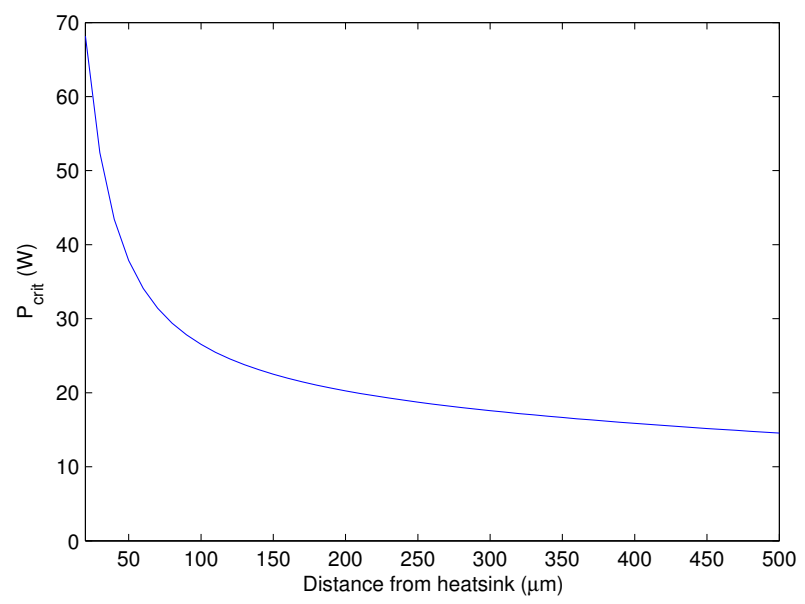

Figure 8.14: Critical pump power required for the temperature change to decrease the excited state lifetime of the laser material modeled for cryogenic cooling.

For Fe:ZnSe, efficient $\mathrm{CW}$ laser operation is only achieved at near cryogenic temperatures. Lifetime data from Adams et al. [81] was fit in order to calculate the derivative of $\frac{1}{\tau}$, Figure 8.15.

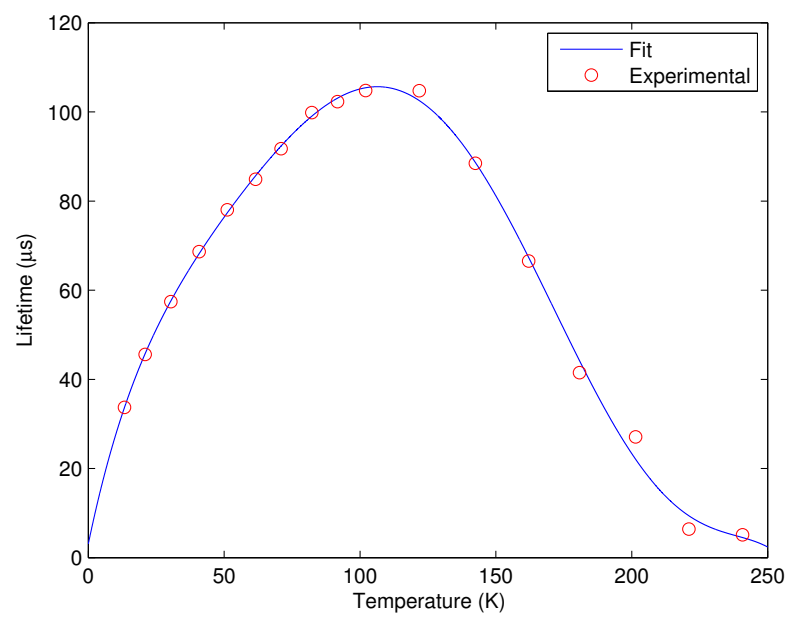

Figure 8.15: 6th order polynomial fit to the experimental data of Adams et al. 
The same modeling as discussed previously was used for modeling of the Fe:ZnSe waveguides. If we now utilize the solution of Equation 8.12 at a critical temperature of $250 \mathrm{~K}$, which is the temperature when the $\mathrm{Fe}^{2+}$ lifetime drops below $1 \mu \mathrm{s}$, we can estimate the performance of Fe:ZnSe waveguides as a function of depth, Figure 8.16.

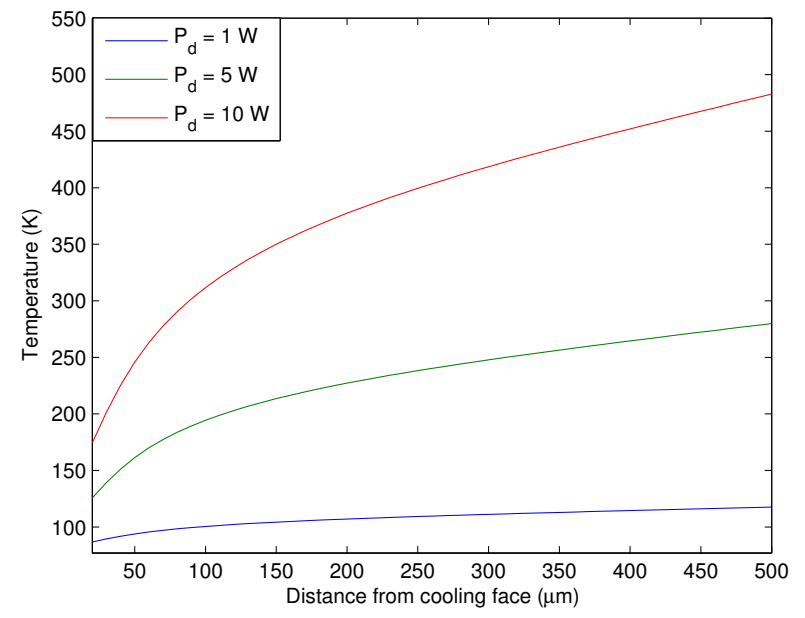

Figure 8.16: Simulated temperature as a function of waveguide depth for Fe:ZnSe.

Figure 8.17 shows the critical power as a function of waveguide depth for a cryogenically cooled Fe:ZnSe waveguide. It can be seen that for a depth of $500 \mu m$, a critical power of $3.8 \mathrm{~W}$ was obtained. Moving to a depth of $20 \mu \mathrm{m}$ increases the critical power to approximately $25 \mathrm{~W}$. 


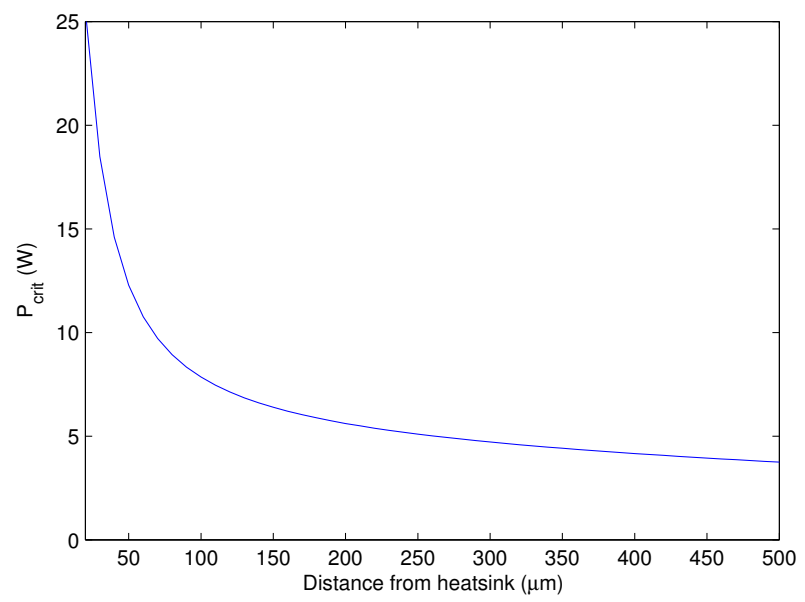

Figure 8.17: Critical power for Fe:ZnSe under cryogenic cooling.

\subsection{Conclusion}

In conclusion, a theoretical model has been established to predict the performance of ULI waveguides. The model calculates the critical power at which the excited state lifetime of the laser medium collapses due to phonon assisted transitions. Table 8.5 summarizes the results of the thermal model for waveguide laser operation. From these results, it can be concluded that waveguides inscribed near the cooling face will reach a critical temperature under higher pumping conditions, thus producing more output power before thermal quenching occurs. For the experimental conditions represented in this work, a critical power was not achieved due to the available pump power. The results presented in Table 8.5 represent the bounds to the experimental data. In addition, several test cases were modeled for larger waveguides up to $200 \mu \mathrm{m}$, with the notion that larger surface area may lead to lower temperature in the core of the waveguide. However, the peak core temperature only dropped by approximately $1 K$. The limiting factor is the poor thermal conductivity of $\mathrm{ZnSe}$, which limits the ability for heat to be extracted from the active region of the waveguide. 
Table 8.5: Summary of modeling results for varying operating temperature and waveguide depth.

\begin{tabular}{|l|c|c|c|c|}
\hline Material & Waveguide Diameter & Operating Temperature & Critical Power & Depth \\
\hline Cr:ZnSe & $40 \mu m$ & $293 \mathrm{~K}$ & $2.1 \mathrm{~W}$ & $500 \mu m$ \\
\hline Cr:ZnSe & $40 \mu m$ & $293 \mathrm{~K}$ & $25 \mathrm{~W}$ & $20 \mu m$ \\
\hline Cr:ZnSe & $40 \mu m$ & $77 \mathrm{~K}$ & $14.5 \mathrm{~W}$ & $500 \mu m$ \\
\hline Cr:ZnSe & $40 \mu m$ & $77 \mathrm{~K}$ & $68 \mathrm{~W}$ & $20 \mu m$ \\
\hline Fe:ZnSe & $40 \mu m$ & $77 \mathrm{~K}$ & $3.7 \mathrm{~W}$ & $500 \mu m$ \\
\hline Fe:ZnSe & $40 \mu m$ & $77 \mathrm{~K}$ & $25.5 \mathrm{~W}$ & $20 \mu m$ \\
\hline
\end{tabular}




\section{CHAPTER IX}

\section{CONCLUSIONS AND FUTURE WORK}

In conclusion, several advancements have been made to the state-of-the-art abilities of ULI devices. ULI was demonstrated using a new algorithm for creating arbitrary geometries in materials. The algorithm used CAD to generate a physical model of the desired waveguide structure. From the CAD drawing, an .stl file can be exported to be used with the algorithm, which generates the physical stage movement. Several complex geometries were inscribed using the algorithAppendix C, including a waveguide splitter and a linear array coupler. ULI waveguide lasers in Cr:ZnSe and Fe:ZnSe were demonstrated utilizing ULI. The waveguides produced record powers of $5 \mathrm{~W}$ for Cr:ZnSe with a slope efficiency of $41 \%$ and $1 W$ for Fe:ZnSe with a slope efficiency of $58 \%$.

In conjunction with the experimental results, a theoretical basis was set forth for determining the maximum possible power attainable from these devices. A thermal model, coupled with a rateequation model, provided the proper theoretical basis for this determination. The determination made from the modeling results was that moving the waveguide closer to the cooling surface of the sample always yielded a higher critical power. For Cr:ZnSe, if the sample was cooled with liquid nitrogen and the $40 \mu \mathrm{m}$ diameter waveguide was inscribed $20 \mu \mathrm{m}$ from the cooling face, a maximum critical pump power of $68 \mathrm{~W}$ was obtained. If a quantum limited slope efficiency of $77 \%$ was assumed, a maximum output power of $\approx 52 \mathrm{~W}$ could be obtained. Likewise, for Fe:ZnSe, a maximum pump power of $25.5 \mathrm{~W}$ was obtained for a $40 \mu \mathrm{m}$ diameter waveguide was inscribed 
$20 \mu \mathrm{m}$ from the cooling face. In practice, these pump powers are well above the cooling capacity of most liquid nitrogen dewars.

Additionally, the first demonstration of a Ho:YAG waveguide laser using ULI was demonstrated. Both single and multimode waveguides were demonstrated, producing output powers of $444 \mathrm{~mW}$ and $1.88 \mathrm{~W}$. Waveguides were also inscribed in a co-doped sample of Yb:Ho:YAG with the goal of direct diode pumping the sample to obtain $2 \mu \mathrm{m}$ emission. The sample produced laser emission under in-band pumping using a $1.9 \mu \mathrm{m}$ laser, but damaged under $940 \mathrm{~nm}$ diode pumping due to thermal stresses.

Future research will be focused on several aspects of this research. Overall, the losses associated with the passive devices need to be decreased in order for the passive devices to be practical. Inscription of Bragg structures inside of active materials can refined with careful control of the ULI parameters. Finally, ULI has shown promise for creating waveguide structures in a variety of nonlinear materials. The preliminary results shown in Appendix A, will be expanded on to generate nonlinear laser devices utilizing ULI technology. 


\section{BIBLIOGRAPHY}

[1] T. H. Maiman, “Stimulated optical radiation in ruby," Nature, vol. 187, no. 4736, pp. 493494, 1960.

[2] U. P. Schieß1 and J. Rohr, " $60{ }^{\circ} C$ lead salt laser emission near 5- $\mu m$ wavelength," Infrared Physics \& Technology”, vol. 40, no. 4, pp. 325 - 328, 1999.

[3] J. Faist, F. Capasso, D. L. Sivco, C. Sirtori, A. L. Hutchinson, and A. Y. Cho, "Quantum cascade laser," Science, vol. 264, no. 5158, pp. 553-556, 1994.

[4] L. DeLoach, R. Page, G. Wilke, S. Payne, and W. Krupke, "Transition metal-doped zinc chalcogenides: Spectroscopy and laser demonstration of a new class of gain media," IEEE J. Quantum Electron., vol. 32, no. 6, pp. 885-895, Jun 1996.

[5] S. McDaniel, D. Hobbs, B. MacLeod, E. Sabatino, P. Berry, K. Schepler, W. Mitchell, and G. Cook, "Cr:ZnSe laser incorporating anti-reflection microstructures exhibiting low-loss, damage-resistant lasing at near quantum efficiency," Opt. Mat. Express, vol. 4, no. 11, pp. 2225-2232, Nov 2014.

[6] A. Sennaroglu, U. Demirbas, N. Vermeulen, H. Ottevaere, and H. Thienpont, "Continuouswave broadly tunable $\mathrm{Cr}^{2+}$ :ZnSe laser pumped by a thulium fiber laser," Opt. Commun., vol. 268, no. 1, pp. 115 - 120, 2006.

[7] V. Fedorov, S. Mirov, A. Gallian, D. Badikov, M. Frolov, Y. Korostelin, V. Kozlovsky, A. Landman, Y. Podmar'kov, V. Akimov, and A. Voronov, "3.77-5.05 $\mu \mathrm{m}$ tunable solid-state lasers based on fe $\hat{2}+-$ doped znse crystals operating at low and room temperatures," Quantum Electronics, IEEE Journal of, vol. 42, no. 9, pp. 907 -917, sept. 2006.

[8] J. Rivera-Iratchet, M. A. de Orúe, and E. E. Vogel, "Vibronic coupling and the near-infrared spectrum of Fe in CdTe and ZnS," Phys. Rev. B, vol. 34, pp. 3992-4001, Sep 1986.

[9] S. B. Mirov, V. Fedorov, D. Martyshkin, I. Moskalev, M. Mirov, and S. Vasilyev, "High Average Power Fe:ZnSe and Cr:ZnSe Mid-IR Solid State Lasers," Advanced Solid State Lasers, p. AW4A.1, 2015. 
[10] D. Martyshkin, V. Fedorov, M. Mirov, I. Moskalev, S. Vasilyev, and S. Mirov, "High average power (35 W) pulsed Fe:ZnSe laser tunable over 3.8-4.2 um," Conference on Lasers and Electro-Optics (CLEO), p. SF1F.2, 2015.

[11] D. B. Keck, “Optical fiber spans 30 years," Lightwave, vol. 17, no. 8, pp. 78-82, 2000.

[12] P. K. Tien, "Light Waves in Thin Films and Ingtegrated Optics," Appl. Opt., vol. 10, pp. 2395-2413, 1970.

[13] H. Ishio, J. Minowa, and K. Nosu, "Review and status of wavelength-division-multiplexing technology and its application," Journal of Lightwave Technology, vol. 2, no. 4, pp. 448-463, 1984.

[14] D. D’Agostino, G. Carnicella, C. Ciminelli, P. Thijs, P. J. Veldhoven, H. Ambrosius, and M. Smit, "Low-loss passive waveguides in a generic inp foundry process via local diffusion of zinc," Opt. Express, vol. 23, no. 19, pp. 25 143-25 157, Sep 2015.

[15] D.-L. Zhang, F. Han, B. Chen, P.-R. Hua, D.-Y. Yu, and E. Y.-B. Pun, "Optical-damageresistant highly er-doped ti:er:linbo strip waveguide," J. Lightwave Technol., vol. 32, no. 1, pp. 135-140, Jan 2014.

[16] K. M. Davis, K. Miura, N. Sugimoto, and K. Hirao, "Writing waveguides in glass with a femtosecond laser," Opt. Lett., vol. 21, no. 21, pp. 1729-1731, Nov 1996.

[17] Optoscribe, "Home,” Optoscribe, Sep 2017. [Online]. Available: http://www.optoscribe.com/

[18] M. Beresna, M. Gecevičius, and P. G. Kazansky, "Polarization sensitive elements fabricated by femtosecond laser nanostructuring of glass," Opt. Mater. Express, vol. 1, no. 4, pp. 783795, Aug 2011.

[19] R. Thomson, S. Campbell, I. Blewett, A. Kar, and D. Reid, "Optical waveguide fabrication in z-cut lithium niobate $\left(\mathrm{LiNbO}_{3}\right)$ using femtosecond pulses in the low repetition rate regime," Applied Physics Letters, vol. 88, no. 11, pp. 111 109-111 109, 2006.

[20] R. R. Thomson, H. T. Bookey, N. D. Psaila, A. Fender, S. Campbell, W. N. Macpherson, J. S. Barton, D. T. Reid, and A. K. Kar, "Ultrafast-laser inscription of a three dimensional fan-out device for multicore fiber coupling applications," Optics Express, vol. 15, no. 18, pp. $11691-11697,2007$.

[21] P. Mitchell, G. Brown, R. R. Thomson, N. Psaila, and A. Kar, "57 channel(19x3) spatial multiplexer fabricated using direct laser inscription," Optical Fiber Communication Conference, p. M3K.5, 2014.

[22] M. Pospiech, M. Emons, A. Steinmann, G. Palmer, R. Osellame, N. Bellini, G. Cerullo, and U. Morgner, "Double waveguide couplers produced by simultaneous femtosecond writing," Opt. Express, vol. 17, no. 5, pp. 3555-3563, 2009. 
[23] D. Choudhury, J. R. Macdonald, and A. K. Kar, "Ultrafast laser inscription: perspectives on future integrated applications," Laser and Photonics Reviews, vol. 8, pp. 219-225, 2014.

[24] R. Mary, D. Choudhury, and A. K. Kar, "Applications of Fiber Lasers for the Development of Compact Photonic Devices,” J. Sel. Top. Quant. Electron., vol. 8, pp. 1-1, 2014.

[25] R. Osellame, S. Taccheo, M. Marangoni, R. Ramponi, P. Laporta, D. Polli, S. D. Silvestri, and G. Cerullo, "Femtosecond writing of active optical waveguides with astigmatically shaped beams," J. Opt. Soc. Am. B, vol. 20, pp. 1559-1567, 2003.

[26] S. Taccheo, G. D. Valle, R. Osellame, G. Cerullo, N. Chiodo, P. Laporta, O. Svelto, A. Killi, U. Morgner, M. Lederer, and D. Kopf, "Er:Yb- doped waveguide laser fabricated by femtosecond laser pulses," Optics Letters, vol. 29, pp. 2626-2628, 2004.

[27] A. G. Okhrimchuk, A. V. Shestakov, I. Khrushchev, and J. Mitchell, "Depressed cladding, buried waveguide laser formed in a YAG: $\mathrm{Nd}^{3+}$ crystal by femtosecond laser writing," Opt. Lett., vol. 30, no. 17, pp. 2248-2250, Sep 2005.

[28] J. R. Macdonald, R. R. Thomson, S. J. Beecher, N. D. Psaila, H. T. Bookey, and A. K. Kar, "Ultrafast laser inscription of near-infrared waveguides in polycrystalline ZnSe," Opt. Lett., vol. 35, no. 23, pp. 4036-4038, Dec 2010.

[29] S. A. McDaniel, A. Lancaster, J. W. Evans, A. K. Kar, and G. Cook, "Power scaling of ultrafast laser inscribed waveguide lasers in chromium and iron doped zinc selenide," Optics Express, vol. 24, no. 4, pp. 3502-3512, 2006.

[30] P. A. Berry, J. R. Macdonald, S. J. Beecher, S. A. McDaniel, K. L. Schepler, and A. K. Kar, "Fabrication and power scaling of a $1.7 \mathrm{~W}$ Cr:ZnSe waveguide laser," Opt. Mater. Express, vol. 3, no. 9, pp. 1250-1258, Sep 2013.

[31] A. Lancaster, G. Cook, S. A. McDaniel, J. Evans, P. Berry, J. D. Shephard, and A. K. Kar, "Mid-infrared laser emission from Fe:ZnSe cladding waveguides," Appl. Phys. Lett., vol. 107, no. 3, 2015.

[32] Femtosecond Laser Micromachining. Springer Science, 2012.

[33] C. Schaffer, A. Brodeur, and E. Mazur, "Laser-induced breakdown and damage in bulk transparent materials induced by tightly focused femtosecond laser pulses," Measurment Science and Technology, vol. 12, no. 11, p. 1784, 2001.

[34] J. R. Macdonald, P. A. Berry, K. L. Schelper, and A. Kar, "Directly written mid-infrared waveguides in zinc selenide,", pp. IF1A-3, 2012.

[35] M. Kaganov, "Relaxation between electrons and the crystalline lattice," Sov. Phys. JETP, vol. 4, pp. 173-178, 1957.

[36] T. O. Parashchuk, "Modeling of Structure, Thermodynamic Properties and Phase Transition Temperatures of II-VI Crystals," Journal of Nano- and Electroic Physics, vol. 8, no. 2, p. 02010, 2016. 
[37] A. Edwards, T. Slykhouse, and H. Drickamer, "The effect of pressure on zinc blends and wurtzite structures," J. Phys. Chem. Solids, vol. 11, pp. 140-148, 1959.

[38] R. W. Stites, S. A. McDaniel, J. O. Barnes, D. M. Krein, J. H. Goldsmith, S. Guha, and G. Cook, "Hot isostatic pressing of transition metal ions into chalcogenide laser host crystals," Opt. Mater. Express, vol. 6, no. 10, pp. 3339-3353, Oct 2016.

[39] P. F. Moulton, "Spectroscopic and laser characteristics of ti: Al 2 o 3," JOSA B, vol. 3, no. 1, pp. 125-133, 1986.

[40] Solid-State Mid-Infrared Laser Souces. Springer, 2003.

[41] Handbook of Solid-State Lasers. Woodhead Publishing, 2013.

[42] J. Vallin, G. Slack, S. Roberts, and A. Hughes, "Infrared Absorption in Some II-VI Compounds Doped with Cr," Phys. Rev. B, vol. 2, p. 4313, 1970.

[43] S. Mirov, V. Fedorov, I. Moskalev, M. Mirov, and D. Martyshkin, "Frontiers of mid-infrared lasers based on transition metal doped II-VI semiconductors," Journal of Luminescence, pp. 268-275, 2013.

[44] Chemical Application of Group Theory. Wiley, 1971.

[45] Okamura, "Measuring mode propagation losses of integrated optical waveguides: a simple method," Applied Optics, vol. 22, pp. 3892-3894, 1983.

[46] G. Hawkins and R. Sherwood, "Cooled infrared filters and dichroics for the james webb space telescope mid-infrared instrument," Appl. Opt., vol. 47, no. 13, pp. C25-C34, May 2008. [Online]. Available: http://ao.osa.org/abstract.cfm?URI=ao-47-13-C25

[47] Szameit, "Two-dimensional solition in cubis fs laser written waveguide arrays in fused silica," Optics Express, vol. 14, pp. 6055-6062, 2006.

[48] Numerical Simulation of Optical Wave Propagation. SPIE Press, 2013.

[49] R. W. Gerchberg, "A practical algorithm for the determination of the phase from image and diffraction plane pictures," Optik, vol. 35, pp. 237-246, 1972.

[50] S. M. Eaton, H. Zhang, P. R. Herman, F. Yoshino, L. Shah, J. Bovatsek, and A. Y. Arai, "Heat accumulation effects in femtosecond laser-written waveguides with variable repetition rate," Opt. Express, vol. 13, no. 12, p. 7317, 2005.

[51] A. H. Nejadmalayeri and P. R. Herman, "Ultrafast laser waveguide writing: lithium niobate and the role of circular polarization and picosecond pulse width," Opt. Lett., vol. 31, no. 202, pp. 2987-2989, 2006.

[52] E. Bricchi, B. G. Klappauf, and P. G. Kazansky, "Form birefringence and negative index change created by femtosecond direct writing in transparent materials," Opt. Lett., vol. 29, no. 1, pp. 119-121, 2004. 
[53] C. M. Liebig, J. Goldstein, S. A. McDaniel, E. Glaze, and D. K. andG. Cook, "Femtosecond laser precipitation of non-centrosymmetric crystals in glasses," Proc.SPIE, vol. 9958, pp. $9958-9958-8,2016$.

[54] A. S. Lipatiev, T. O. Lipateva, S. V. Lotarev, A. G. Okhrimchuk, A. S. Larkin, M. Y. Presnyakov, and V. N. Sigaev, "Direct laser writing of labgeo5 crystal-in-glass waveguide enabling frequency conversion," Crystal Growth \& Design, vol. 17, no. 9, pp. 4670-4675, 2017.

[55] R. W. Birkmire, "Compound polycrystalline solar cells:: Recent progress and y2k perspective," Solar Energy Materials and Solar Cells, vol. 65, no. 1, pp. 17 - 28, 2001.

[56] Y. Shimotsuma, P. G. Kazansky, J. Qiu, and K. Hirao, "Self-organized nanogratings in glass irradiated by ultrashort light pulses," Phys. Rev. Lett., vol. 91, p. 247405, Dec 2003.

[57] V. R. Bhardwaj, E. Simova, P. P. Rajeev, C. Hnatovsky, R. S. Taylor, D. M. Rayner, and P. B. Corkum, "Optically produced arrays of planar nanostructures inside fused silica," Phys. Rev. Lett., vol. 96, p. 057404, Feb 2006.

[58] R. M. Measures, Laser remote sensing: fundamentals and applications. Wiley, 1984.

[59] R. Hibst and U. Keller, "Experimental studies of the application of the er: Yag laser on dental hard substances: I. measurement of the ablation rate," Lasers in Surgery and Medicine, vol. 9, no. 4, pp. 338-344, 1989.

[60] B. Bernhardt, E. Sorokin, P. Jacquet, R. Thon, T. Becker, I. Sorokina, N. Picqué, and T. Hänsch, "Mid-infrared dual-comb spectroscopy with $2.4 \mu \mathrm{m}$ cr 2+: Znse femtosecond lasers," Applied Physics B: Lasers and Optics, vol. 100, no. 1, pp. 3-8, 2010.

[61] J. Evans, P. Berry, and K. Schepler, "A Passively Q-switched, CW-pumped Fe:ZnSe Laser," IEEE J. Quantum Electron., vol. 50, no. 3, pp. 204-2008, March 2014.

[62] V. Fedorov, D. Martyshkin, M. Mirov, I. S. Moskalev, S. Vasilyev, J. Peppers, S. B. Mirov, and V. P. Gapontsev, "Fe-doped II-VI Mid-Infrared Laser Materials for the 3 to $8 \mu \mathrm{m}$ Region," Conference on Lasers and Electro-Optics (CLEO), p. JM4K.2, 2013.

[63] J. W. Evans, P. A. Berry, and K. L. Schepler, "840 mW continuous-wave Fe:ZnSe laser operating at 4140 nm," Opt. Lett., vol. 37, no. 23, pp. 5021-5023, Dec 2012. [Online]. Available: http://ol.osa.org/abstract.cfm?URI=ol-37-23-5021

[64] I. Moskalev, S. Mirov, M. Mirov, S. Vasilyev, V. Smolski, A. Zakrevskiy, and V. Gapontsev, “140 w cr:znse laser system,” Opt. Express, vol. 24, no. 18, pp. 21 090-21 104, Sep 2016.

[65] D. Siche and H. Hartmann, "Source-material dependent growth limitations in unseeded dissociative sublimation of ZnSe," J. Mater. Sci., vol. 31, no. 23, pp. 6171-6175, 1996.

[66] R. Nitsche, H. Bölsterli, and M. Lichtensteiger, "Crystal growth by chemical transport reactionsi: Binary, ternary, and mixed-crystal chalcogenides," Journal of Physics and Chemistry of Solids, vol. 21, no. 3-4, pp. 199-205, 1961. 
[67] P. Berry, "Versatile Chromium-Doped Zinc Selenide Infrared Laser Sources," Ph.D. dissertation, University of Dayton, 2010.

[68] J. R. Sparks, R. He, N. Healy, M. Krishnamurthi, A. C. Peacock, P. J. A. Sazio, V. Gopalan, and J. V. Badding, "Zinc Selenide Optical Fibers," Adv. Mater, vol. 23, no. 14, pp. 1647$1651,2011$.

[69] J. R. Macdonald, S. J. Beecher, P. A. Berry, G. Brown, K. L. Schepler, and A. K. Kar, "Efficient mid-infrared Cr:ZnSe channel waveguide laser operating at 2486 nm," Opt. Lett., vol. 38, no. 13, pp. 2194-2196, Jul 2013.

[70] S. A. McDaniel, P. A. Berry, K. L. Schepler, J. R. Macdonald, S. J. Beecher, and A. K. Kar, "Gain-switched operation of ultrafast laser inscribed waveguides in Cr:ZnSe," Proc. SPIE, vol. 9342, pp. 93 420E-93 420E-8, 2015.

[71] E. Ghahramani, D. Moss, and J. Sipe, "Full-band-structure calculation of first, second, and third-harmonic optical response coefficients of ZnSe, ZnTe, and CdTe," Phys. Rev. B, vol. 43, no. 12 , p. $9700,1991$.

[72] J. R. Macdonald, S. J. Beecher, P. A. Berry, K. L. Schepler, and A. K. Kar, "Compact midinfrared Cr:ZnSe channel waveguide laser," Appl. Phys. Lett., vol. 102, no. 16, p. 161110 , 2013.

[73] S. B. Mirov, V. V. Fedorov, K. Graham, I. S. Moskalev, V. V. Badikov, and V. Panyutin, "Erbium fiber laser-pumped continuous-wave microchip $\mathrm{Cr}^{2+}: \mathrm{ZnS}$ and $\mathrm{Cr}^{2+}: \mathrm{ZnSe}$ lasers," Opt. Lett., vol. 27, no. 11, pp. 909-911, Jun 2002.

[74] H. Jelínková, M. E. Doroshenko, M. Jelínek, D. Vyhlídal, J. Šulc, M. Němec, V. Kubeček, Y. A. Zagoruiko, N. O. Kovalenko, A. S. Gerasimenko, V. M. Puzikov, and V. K. Komar, "Fe:ZnSe laser oscillation under cryogenic and room temperature," Proc. SPIE, vol. 8599, pp. 85 990E-85 990E-7, 2013. [Online]. Available: http://dx.doi.org/10.1117/12.2003840

[75] J. W. Evans, P. A. Berry, and K. L. Schepler, “A broadly tunable continuous-wave Fe:ZnSe laser." Proc. SPIE, vol. 8599, no. 11, 2013.

[76] G. A. Slack, F. S. Ham, and R. M. Chrenko, "Optical Absorption of Tetrahedral $F e^{2+}\left(3 d^{6}\right)$ in Cubic ZnS, CdTe, and $\mathrm{MgAl}_{2} \mathrm{O}_{4}$," Phys. Rev., vol. 152, pp. 376-402, Dec 1966. [Online]. Available: http://link.aps.org/doi/10.1103/PhysRev.152.376

[77] D. Findlay and R. Clay, "The measurement of internal losses in 4-level lasers," Physics Letters, vol. 20, no. 3, pp. $277-278,1966$.

[78] W. W. Rigrod, "Saturation effects in high-gain lasers," Journal of Applied Physics, vol. 36, no. 8, pp. 2487-2490, 1965.

[79] R. M. Wood, Laser-induced damage of optical materials. CRC Press, 2003.

[80] A. Siegman, Lasers. University Science Books, 1986. 
[81] J. J. Adams, C. Bibeau, R. H. Page, D. M. Krol, L. H. Furu, and S. A. Payne, "4.0-4.5- $\mu \mathrm{m}$ lasing of Fe:ZnSe below $180 \mathrm{~K}$, a new mid-infrared laser material,” Opt. Lett., vol. 24, no. 23, pp. 1720-1722, Dec 1999.

[82] P. A. Berry and K. L. Schepler, "High-power, widely-tunable cr2+:znse?master oscillator power amplifier systems," Opt. Express, vol. 18, no. 14, pp. 15 062-15 072, Jul 2010.

[83] S. A. McDaniel, P. A. Berry, and K. L. Schepler, "Gain-switched single-pass Cr:ZnSe amplifier," Proc.SPIE, vol. 8599, pp. 8599 - 8599 - 8, 2013.

[84] E. Lippert, S. Nicolas, G. Arisholm, K. Stenersen, and G. Rustad, "Midinfrared laser source with high power and beam quality," Appl. Opt., vol. 45, no. 16, pp. 3839-3845, Jun 2006.

[85] G. Brown, R. R. Thomson, A. K. Kar, N. D. Psaila, and H. T. Bookey, "Ultrafast laser inscription of bragg-grating waveguides using the multiscan technique," Opt. Lett., vol. 37, no. 4, pp. 491-493, Feb 2012.

[86] V. Bharadwaj, A. Courvoisier, T. T. Fernandez, R. Ramponi, G. Galzerano, J. Nunn, M. J. Booth, R. Osellame, S. M. Eaton, and P. S. Salter, "Femtosecond laser inscription of bragg grating waveguides in bulk diamond," Opt. Lett., vol. 42, no. 17, pp. 3451-3453, Sep 2017.

[87] S. Antipov, M. Ams, R. J. Williams, E. Magi, M. J. Withford, and A. Fuerbach, "Direct infrared femtosecond laser inscription of chirped fiber bragg gratings," Opt. Express, vol. 24, no. 1, pp. 30-40, Jan 2016.

[88] K. Trauner, N. Nishioka, and D. Patel, "Pulsed holmium: yttrium-aluminum-garnet (Ho: YAG) laser ablation of fibrocartilage and articular cartilage," The American journal of sports medicine, vol. 18, no. 3, pp. 316-320, 1990.

[89] I. Cernavin, "A comparison of the effects of Nd: YAG and Ho: YAG laser irradiation on dentine and enamel," Australian dental journal, vol. 40, no. 2, pp. 79-84, 1995.

[90] H. Cankaya, M. N. Cizmeciyan, E. Beyatli, A. T. Gorgulu, A. Kurt, and A. Sennaroglu, "Injection-seeded, gain-switched tunable Cr:ZnSe laser," Opt. Lett., vol. 37, no. 2, pp. 136-138, Jan 2012. [Online]. Available: http://ol.osa.org/abstract.cfm?URI=ol-37-2-136

[91] P. Budni, L. Pomeranz, M. Lemons, C. Miller, J. Mosto, and E. Chicklis, "Efficient midinfrared laser using 1.9- $\mu \mathrm{m}$-pumped Ho: YAG and ZnGeP 2 optical parametric oscillators," JOSA B, vol. 17, no. 5, pp. 723-728, 2000.

[92] T. Y. Fan, G. Huber, R. L. Byer, and P. Mitzscherlich, "Spectroscopy and diode laser-pumped operation of Tm,Ho:YAG," IEEE Journal of Quantum Electronics, vol. 24, no. 6, pp. 924933, June 1988.

[93] R. Remski and D. Smith, "Temperature dependence of pulsed laser threshold in YAG: Er 3+, Tm 3+, Ho+," IEEE Journal of Quantum Electronics, vol. 6, no. 11, pp. 750-751, 1970. 
[94] C. D. Nie, S. Bera, and J. A. Harrington, "Growth of single-crystal YAG fiber optics," Opt. Express, vol. 24, no. 14, pp. 15 522-15 527, Jul 2016.

[95] H. Kim, R. S. Hay, S. A. McDaniel, G. Cook, N. G. Usechak, A. M. Urbas, K. N. Shugart, H. Lee, A. H. Kadhim, D. P. Brown, B. Griffin, G. E. Fair, R. G. Corns, S. A. Potticary, F. K. Hopkins, K. L. Averett, D. E. Zelmon, T. A. Parthasarathy, and K. A. Keller, "Lasing of surface-polished polycrystalline ho: Yag (yttrium aluminum garnet) fiber," Opt. Express, vol. 25, no. 6, pp. 6725-6731, Mar 2017.

[96] T. Sumiyoshi, H. Sekita, T. Arai, S. Sato, M. Ishihara, and M. Kikuchi, "High-power continuous-wave 3- and 2- $\mu \mathrm{m}$ cascade Ho3+:ZBLAN fiber laser and its medical applications," IEEE Journal of Selected Topics in Quantum Electronics, vol. 5, no. 4, pp. 936-943, 1999.

[97] H. P. J.Y. Allain, M. Monerie, "High-efficiency CW thulium-sensitised holmium-doped fluoride fibre laser operating at $2.04 \mu \mathrm{m}$," Electronics Letters, vol. 27, pp. 1513-1515(2), August 1991.

[98] A. Guhur and S. D. Jackson, "Efficient holmium-doped fluoride fiber laser emitting $2.1 \mu \mathrm{m}$ and blue upconversion fluorescence upon excitation at $2 \mu \mathrm{m}$," Opt. Express, vol. 18, no. 19, pp. $20164-20169$, Sep 2010.

[99] K. Oh, T. F. Morse, P. M. Weber, A. Kilian, and L. Reinhart, "Continuous-wave oscillation of thulium-sensitized holmium-doped silica fiber laser," Opt. Lett., vol. 19, no. 4, pp. 278-280, Feb 1994.

[100] N. Ter-Gabrielyan, V. Fromzel, X. Mu, H. Meissner, and M. Dubinskii, "Resonantly pumped single-mode channel waveguide Er:YAG laser with nearly quantum defect limited efficiency," Opt. Lett., vol. 38, no. 14, pp. 2431-2433, Jul 2013.

[101] S. A. McDaniel, P. A. Berry, G. Cook, D. Zelmon, S. Meissner, H. Meissner, and X. Mu, "CW and passively Q-Switched operation of a Ho:YAG waveguide laser ," Optics and Laser Technology, vol. 91, pp. 1-6, 2017.

[102] A. Rodenas, A. Benayas, J. R. Macdonald, J. Zhang, D. Y. Tang, D. Jaque, and A. K. Kar, "Direct laser writing of near-IR step-index buried channel waveguides in rare earth doped YAG,” Opt. Lett., vol. 36, no. 17, pp. 3395-3397, Sep 2011.

[103] Y. Ren, G. Brown, A. Ródenas, S. Beecher, F. Chen, and A. K. Kar, "Mid-infrared waveguide lasers in rare-earth-doped YAG," Opt. Lett., vol. 37, no. 16, pp. 3339-3341, Aug 2012.

[104] D. Lancaster, S. Gross, H. Ebendorff-Heidepriem, K. Kuan, T. Monro, M. Ams, A. Fuerbach, and M. Withford, "Fifty percent internal slope efficiency femtosecond direct-written tm 3+: Zblan waveguide laser," Opt. Lett., vol. 36, no. 9, pp. 1587-1589, 2011.

[105] A. H. Nejadmalayeri, P. R. Herman, J. Burghoff, M. Will, S. Nolte, and A. Tünnermann, "Inscription of optical waveguides in crystalline silicon by mid-infrared femtosecond laser pulses," Opt. Lett., vol. 30, no. 9, pp. 964-966, May 2005. 
[106] M. Schellhorn and A. Hirth, "Modeling of intracavity-pumped quasi-three-level lasers," IEEE Journal of Quantum Electronics, vol. 38, no. 11, pp. 1455-1464, Nov 2002.

[107] S. McDaniel, F. Thorburn, A. Lancaster, R. Stites, G. Cook, and A. Kar, "Operation of ho:yag ultrafast laser inscribed waveguide lasers," Appl. Opt., vol. 56, no. 12, pp. 3251-3256, Apr 2017.

[108] B. Antipenko, A. Zabaznov, A. Nikitichev, V. A. P. mennyı̆, A. Stavrov, Y. S. Tipenko, V. B. Tsvetkov, and I. A. Shcherbakov, "Periodic-pulse operation of holmium lasers using yag and ysgg crystals," Quantum Electronics, vol. 23, no. 12, p. 997, 1993.

[109] T. Rothacher, W. Lthy, and H. Weber, "Diode pumping and laser properties of Yb:Ho:YAG ," Optics Communications, vol. 155, no. 13, pp. 68 - 72, 1998.

[110] R. W. Stites and T. R. Harris, "Spectroscopic investigation of Yb,Ho,Pr:YAG as a $3 \mu m$ laser source,” Proc. SPIE, vol. 9726, pp. 97 2610-97 261O-6, 2016.

[111] R. Wälti, W. Lüthy, H.P.Weber, S. Y. Rusanow, A. Yakovlev, A. Zagumenyi, and I. S. A. Umyskov, "Yb3+ Ho3+ energy exchange mechanisms in Yb: Ho: YAG crystals for $2 \mu \mathrm{m}$ or $540 \mathrm{~nm}$ lasing," Journal of quantitative spectroscopy and radiative transfer, vol. 54, no. 4, pp. 671-681, 1995.

[112] J. W. Evans, P. A. Berry, and K. L. Schepler, "840 mW continuous-wave Fe:ZnSe laser operating at 4140 nm," Opt. Lett., vol. 37, no. 23, pp. 5021-5023, 2012.

[113] P. A. Berry and K. Schepler, "High-power, widely tunable cr2+:znse laser," Conference on Lasers and Electro-Optics, p. CMDD5, 2010.

[114] I. T. Sorokina, E. Sorokin, S. Mirov, V. Fedorov, V. Badikov, V. Panyutin, and K. I. Schaffers, "Broadly tunable compact continuous-wave cr :zns laser," Opt. Lett., vol. 27, no. 12, pp. 1040-1042, Jun 2002.

[115] J. Kernal, V. V. Fedorov, A. Gallian, S. B. Mirov, and V. V. Badikov, "3.9-4.8 $\mu m$ gainswitched lasing of Fe:ZnSe at room temperature," Opt. Express, vol. 13, no. 26, pp. 10608 10615 , Dec 2005.

[116] S. Lamrini, P. Koopmann, M. Schäfer, K. Scholle, and P. Fuhrberg, "Efficient high-power ho: Yag laser directly in-band pumped by a gasb-based laser diode stack at $1.9 \mu \mathrm{m}$," Applied Physics B, vol. 106, no. 2, pp. 315-319, 2012.

[117] H. Bookey, R. Thomson, N. D. Psaila, N. C. A. Kar, R. Osellame, and G. Cerullo, "Femtosecond laser inscription of low insertion loss waveguides in z-cut lithium niobate," Photonics Technology Letters, vol. 19, no. 12, p. 892894, 2007.

[118] B. Denker and E. Shklovsky, Handbook of Solid-State Lasers. Elsevier, 2013.

[119] A. M. Zaitsev, Optical Properties of Diamond. Springer Science, 2013. 
[120] I. V. Mochalov, "Laser and nonlinear properties of the potassium gadolinium tungstate laser crystal kgd(wo4)2:nd3+ (kgw:nd),", vol. 36, no. 6, pp. 1660-1669, 1997.

[121] M. N. Islam, Raman Amplifiers for Telecommunications 1: Physical Principles. Springer Science, 2003.

[122] S. Eaton, C. Merchant, R. Iyer, A. Zilkie, A. Helmy, J. Aitchison, P. Herman, R. M. D. Kraemer, and C. H. et al., "Raman gain from waveguides inscribed in kgd (wo 4) 2 by high repetition rate femtosecond laser," Applied Physics Letters, vol. 92, no. 8, p. 081105, 2008.

[123] J. Piper and H. M. P. et al., "Crystalline raman lasers," Selected Topics in Quantum Electronics, IEEE Journal of, vol. 13, no. 3, pp. 692-704, 2007. 


\section{APPENDIX A}

\section{NONLINEAR DEVICES}

\section{A.1 Introduction}

In addition to solid state laser materials, ULI has been shown to be a viable option for producing guided-wave structures in a host of nonlinear materials[102][117][51]. Due to the dependence on intensity, confining the optical field is beneficial for nonlinear processes. Generation of $\chi^{(3)}$ processes, namely Raman generation in crystalline materials, is an ideal embodiment of ULI waveguide technology.

\section{A.2 Nonlinear Processes}

The Raman effect describes the material interaction where photons scattered by the material have a different energy than the incident photon. The photons incident on the material lose energy, referred to as Stokes shifted, thus the wavelength was shifted to a longer wavelength. Conversely, the anti-Stokes allows the incident photon to gain energy, thus decreases the wavelength. In solid materials, these Stokes and anti-Stokes shifts correspond to lattice vibrations of the material. A list of Raman shifts for a given subset of materials is shown in Table A1[118]. 
Table A1: Raman material parameters

\begin{tabular}{|l|c|r|}
\hline Material & Raman Shift $\left(\mathrm{cm}^{-1}\right)$ & Raman Gain @ $1 \mu m(\mathrm{~cm} / \mathrm{GW})$ \\
\hline Diamond & 1332 & 13.5 \\
$\mathrm{Ba}\left(\mathrm{NO}_{3}\right)_{2}$ & 1047 & 11 \\
Fused Silica & 440 & $3.2 \times 10^{-3}$ \\
KTA & 234 & 2 \\
KGW & 901 & 3.5 \\
\hline
\end{tabular}

If we assume a plane wave interaction with a medium, in addition to a slowly varying envelope approximation, the wave equation can be written as

$$
2 i k \frac{d A}{d z} e^{i k z}=-\omega^{2} \mu_{0} P^{N L}
$$

In Equation A.1, $P^{N L}$ in the induced nonlinear polarization, $k$ is the wavevector of light, $\omega$ is the frequency of light, $z$ is the distance of propagation, $A$ is the complex amplitude of the optical field, and $\mu_{0}$ is the electric permeability of vacuum. If we substitute the nonlinear polarization, we can obtain

$$
\frac{d A_{s}}{d z}=i \frac{3 \omega_{s}}{4 n_{s} c} \chi_{Q S}^{(3)} A_{P} A_{P}^{*} A_{s}
$$

where $\chi_{Q S}^{(3)}$ is the contracted notion, which is expanded in

$$
\chi_{Q S}^{(3)}=\chi_{S}^{(3)}\left(\omega_{s} ; \omega_{p},-\omega_{P}, \omega_{s}\right)
$$

Notably, there is no dependence on the factor of $e^{i k z}$, thus Raman processes are automatically phase matched for Stokes generation. When the pump and Stokes inputs to the system have an energy spacing that matches a vibrational excitation, then $\chi_{Q}^{(3)}$ is a negative imaginary number

$$
\chi_{Q S}^{(3)}=-i\left|\chi_{Q S}^{(3)}\right|
$$

If we substitute Equation A.4 back in to Equation A.1, we obtain

$$
\frac{d A_{s}}{d z}=\frac{3 \omega_{s}}{4 n_{s} c}\left|\chi_{Q S}^{(3)}\right|\left|A_{P}\right|^{2} A_{s}
$$


In the undepleted pump approximation, the solution to Equation A.5 becomes

$$
A_{s}(z)=A_{s}(0) e^{g I_{p} z / 2} .
$$

where

$$
g=\frac{3 \omega_{s}}{\varepsilon_{0} n_{s} n_{p} c^{2}} \chi_{Q S}^{(3)}
$$

On resonance, the Stokes field experiences exponential gain if the frequency difference is $\omega_{\Delta}=$ $\omega_{P}-\omega_{s}$. With the basic wave equation set forth, we can concentrate on the generation of the Stokes field for generation of Mid-IR emission. The generation of the Stokes field is governed by a set of coupled differential equation, Equation A.8, for co-propagating pump and Stokes emission. A counter-propagating can be modeled by changing the sign of $\frac{d I_{P}}{d z}$.

$$
\begin{array}{r}
\frac{d I_{s}}{d z}=\frac{\gamma}{\omega_{P}} I_{s} I_{P}, \\
\frac{d I_{P}}{d z}=-\frac{\gamma}{\omega_{s}} I_{s} I_{P}, \\
\gamma=\frac{3 \omega_{s} \omega_{P}}{4 n_{s} n_{P} \varepsilon c^{2}}\left|\operatorname{Im}\left(\chi_{Q S}^{(3)}\right)\right| .
\end{array}
$$

These set of equations can be solved numerically given a specific set of material parameter.

\section{A.3 Materials of Mid-IR Stokes Generation}

Several factors have to be taken into account for a material to be suitable of Mid-IR generation. First, the material must be transparent in the region of interest. This criteria rules out several materials listed in Table A1. Silica, assuming IR grade silica, does not transmit past $3.4 \mu \mathrm{m}$. However, there is significant material absorption when moving past $2.6 \mu \mathrm{m}$. Barium Nitrate $\mathrm{Ba}\left(\mathrm{NO}_{3}\right)_{2}$ and Potassium Titanule Aresenate (KTA) start to absorb around $2 \mu \mathrm{m}$. This leaves Diamond and Potassium Gadolinium Tungstate (KGW). Diamond has many favorable properties for optical applications including damage threshold, Far-IR transmission, large Raman gain, and high thermal conductivity[119]. However, multiphonon absorption limits the usability of Diamond in the 2.5 $-6 \mu m$ range[119]. A more appropriate material to use would be KGW, Table A2[120]. 
Table A2: Material parameters of KGW

\begin{tabular}{|l|c|}
\hline Property & Value Along [100] \\
\hline Knoop Hardness $\left(\mathrm{kg} / \mathrm{mm}^{2}\right)$ & 370 \\
Young's modulus $(\mathrm{GPa})$ & 115.8 \\
Thermal Expansion Coeff. $\left(\times 10^{-6} \mathrm{~K}^{-1}\right)$ & 4 \\
Thermal Conductivity $\left(\mathrm{Wm}^{-1} \mathrm{~K}^{-1}\right)$ & 2.6 \\
Index @1.06 $\mu \mathrm{m}$ Along [100] & 2.03 \\
Raman Gain @ $(1.06 \mu \mathrm{m})(\mathrm{cm} / \mathrm{GW})$ & 6.2 \\
\hline
\end{tabular}

However, KGW has never been demonstrated to have gain past $1.3 \mu \mathrm{m}$. From Equation A.7, we know that the Raman gain relies on the nonlinear susceptibility $\chi_{Q S}^{(3)}$, where

$$
\begin{aligned}
\chi_{Q S}^{(3)}=\chi_{S}^{(3)}\left(\omega_{s} ; \omega_{p},-\omega_{P}, \omega_{s}\right) & =\frac{N \varepsilon_{0}}{2 m} \frac{\left(\omega_{R}^{2}-\omega_{\Delta}^{2}\right)}{\left(\omega_{R}^{2}-\omega_{\Delta}^{2}\right)^{2}+\Gamma^{2} \omega_{\Delta}^{2}}\left(\left.\frac{d \alpha}{d Q}\right|_{0}\right)^{2} \\
& -i \frac{N \varepsilon_{0}}{2 m} \frac{\Gamma \omega_{\Delta}}{\left(\omega_{R}^{2}-\omega_{\Delta}^{2}\right)^{2}+\Gamma^{2} \omega_{\Delta}^{2}}\left(\left.\frac{d \alpha}{d Q}\right|_{0}\right)^{2} .
\end{aligned}
$$

Inserting Equation A.9 into Equation A.7, the dependence of the Raman gain on the frequency of the light utilized can be seen. It follows that the Raman scattering cross-section and Raman gain are proportional to $1 / \lambda^{4}[121]$ for the Stokes wavelength. Waveguides have been demonstrated in $\mathrm{KGW}$ [122]. However, these waveguides were limited in performance to only $5 \%$ conversion efficiency, which given a $200 n J$ pulse, only correlates to $10 n J$ of output energy in the first Stokes. Additionally, the high intensity needed for efficient Raman conversion limited the total power that could be coupled into the waveguide due to end facet damage. The parameters listed previously in Table A1 can now be coupled with Equation A.8 to obtain a rudimentary model for predicting Raman conversion in KGW. However, the missing piece of information is the gain coefficient at the Stokes wavelength in the Mid-IR. We can apply a $1 / \lambda^{4}$ fit to the published values of the gain coefficient at known wavelength: $11.8 \mathrm{~cm} / \mathrm{GW}$ at $532 \mathrm{~nm}$ [123], $3.5 \mathrm{~cm} / \mathrm{GW}$ at $1064 \mathrm{~nm}$ [123], and $1.5 \mathrm{~cm} / \mathrm{GW}$ at $1300 \mathrm{~nm}$. Figure A1 shows the conversion into the first Stokes under $2 \mu \mathrm{m}$ pumping. In order to obtain Figure A.5, it was assumed that a $200 \mathrm{~mW}$ average power, $100 \mathrm{~ns}$ 
pulse was incident on a $50 \mu \mathrm{m}$ diameter waveguide. Additionally, it was assumed to have a gain coefficient of $0.01 \times 10^{-9} \mathrm{~cm} / \mathrm{GW}$, which is about $10 \times$ lower than the estimated values of Raman gain for KGW, and no propagation losses. It can be seen that the pump converts to the first Stokes in $5 \mathrm{~cm}$.

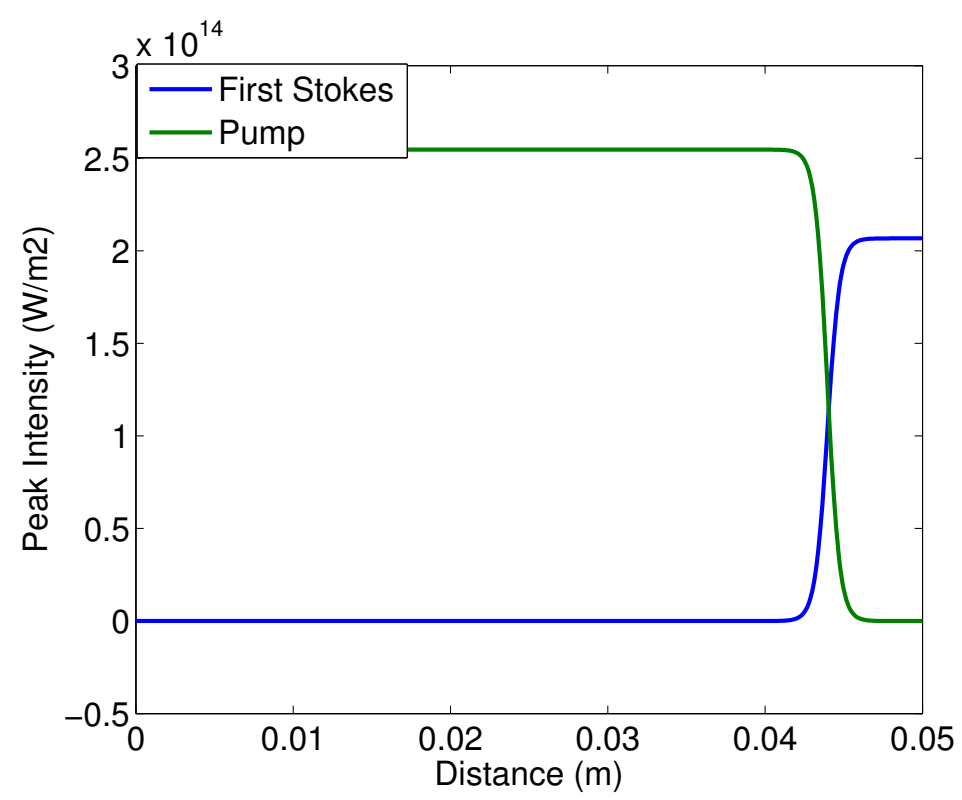

Figure A1: Generation of the first Stokes from KGW using a $2 \mu m$ pump laser.

\section{A.4 Recent Progress}

Preliminary fabrication of a couple nonlinear sources has been attempted. Waveguides have been inscribed in a sample of $\mathrm{KGW}$ and a sample of gallium phosphide $(\mathrm{GaP})$. Figure A2 shows the first attempted femtosecond modification of GaP. The dark ovals that appear in the figure are end facet damage due to the inscription beam exiting the sample upon completion of the translation movement. However, just underneath these dark ovals are circular regions, which are actually ULI 
modification of the GaP. Unfortunately, poor surface quality and cracking due to the ULI process prevented any characterization measurements from yielding reasonable results. Poor coupling due to the surface quality in addition to high losses due to cracking prevented even rudimentary coupling into the modification region.

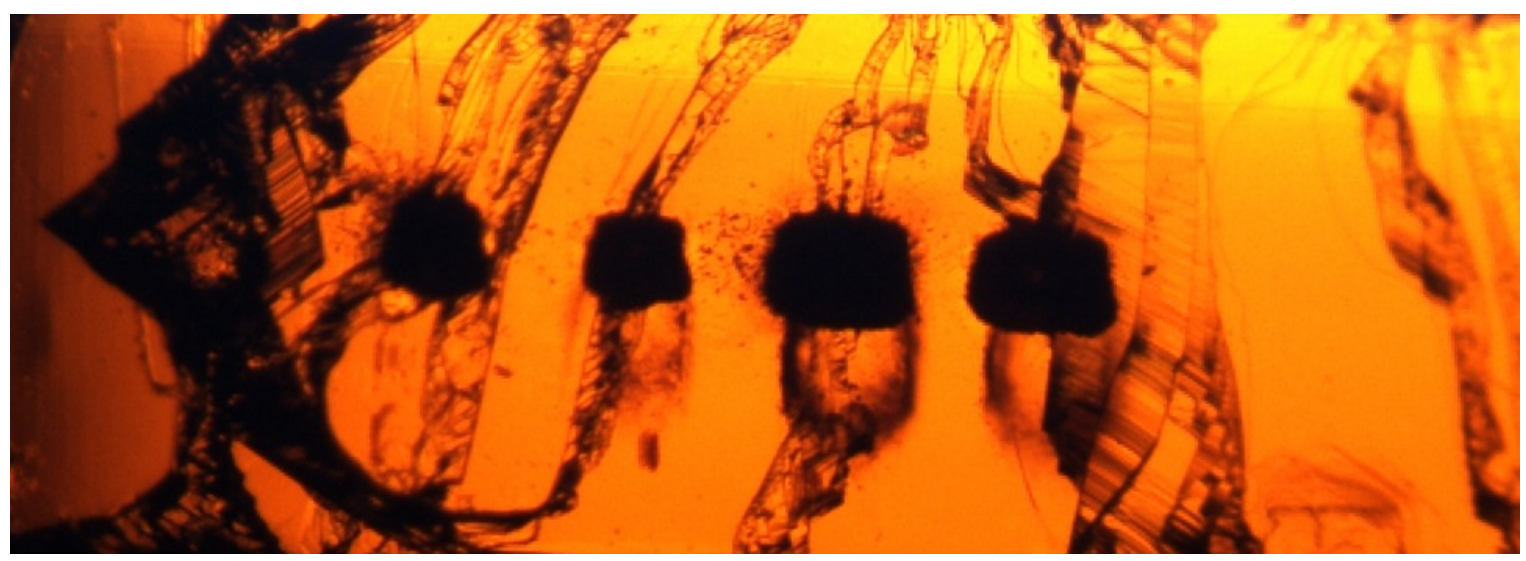

Figure A2: First attempted femtosecond modification of GaP.

Inscription in $\mathrm{KGW}$ was also tested, Figure A3. Again, severe cracking was present in the sample, shown in Figure A3 in the lower left. Despite the severe cracking, a set of waveguides were inscribed without cracking in the sample. The waveguides were inscribed in the sample of $\mathrm{KGW}$ at a repetition rate of $200 \mathrm{kHz}$, an average power of $220 \mathrm{~mW}$, a pulse width of $380 \mathrm{fs}$, and a translation velocity of $10 \mathrm{~mm} / \mathrm{s}$. However, the waveguide elements were extremely elongated, such that the waveguide was completely opaque. No light was observed to guide through the sample, yet the presence of material modification shows promise for creating waveguides with optimized parameters. 


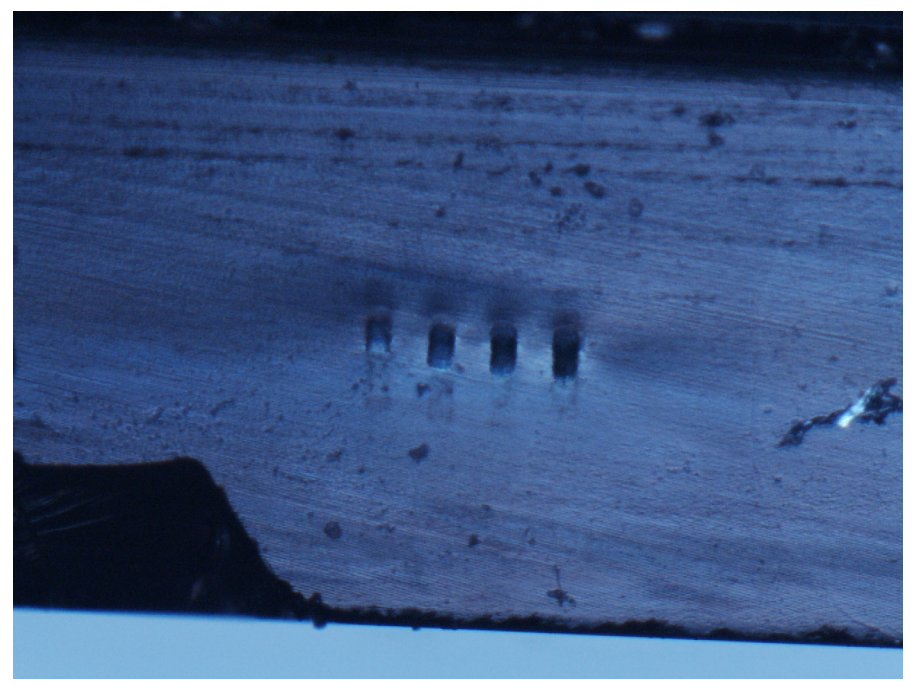

Figure A3: First attempted femtosecond modification of KGW. 


\section{APPENDIX B}

\section{BRAGG GRATING THEORY}

In order to calculate the gratings needed to produce a narrow linewidth laser, a methodology needs to be set forth for determining the entire system response of the setup shown in Figure 6.22. For counter propagating waves inside of a grating, the amplitudes of both fields are given by

$$
\begin{array}{r}
\frac{d a_{1}}{d z}=-i \beta_{1} a_{1}-i \kappa_{12}^{+1} a_{2} e^{i K z}, \\
\frac{d a_{2}}{d z}=-i \beta_{2} a_{2}-i \kappa_{12}^{-1} a_{1} e^{-i K z} .
\end{array}
$$

In the above equations, $a_{1}$ and $a_{2}$ are the field amplitudes, $\beta_{1}$ and $\beta_{2}$ are the propagation constants of the forward and backwards propagating waves, $\kappa$ is the coupling constant between the two waves and $K=2 \pi / \Lambda$, where $\Lambda$ is the grating period. These coupled differential equations now must be decoupled in order to solve to each field individually. To do this, the derivative of the first equation is taken

$$
\frac{d^{2} a_{1}}{d z^{2}}=-i \beta_{1} \frac{d a_{1}}{d z}-i \kappa_{12}^{+1} a_{2} e^{i K z} \frac{d a_{2}}{d z}+\kappa_{12}^{+1} K a_{2} e^{i K z}
$$

Now, the equation for $\frac{d^{2} a_{1}}{d z^{2}}$ can be substituted into Equation B.2. After some algebra, the second order differential equation is shown in

$$
\frac{d^{2} a_{1}}{d z^{2}}+i\left(\beta_{1}+\beta_{2}-K\right) \frac{d a_{1}}{d z}+\left(\kappa_{12}^{+1} \kappa_{12}^{-1}-\beta_{1} \beta_{2}+K \beta_{1}\right) a_{1}=0
$$


The solution of this equation is of the form shown in

$$
a_{1}=\left[C_{1} e^{i \gamma z}+C_{2} e^{-i \gamma z}\right] e^{-i\left(\bar{\beta}-\frac{K}{2}\right) z} .
$$

In Equation B.4, the constants are given as

$$
\begin{array}{r}
\gamma=\sqrt{\delta^{2}+\kappa^{2}}, \\
\kappa=\sqrt{\kappa_{12}^{+1} \kappa_{12}^{-1}}, \\
\delta=\frac{\beta_{2}-\beta_{1}-K}{2}, \\
\bar{\beta}=\frac{\beta_{2}+\beta_{1}}{2}, \\
\kappa_{12}=\left\langle E_{1}\left|\frac{k_{0}^{2}\left(n_{12}^{2}-n_{2}^{2}\right)}{2 \beta_{1}}\right| E_{2}\right\rangle .
\end{array}
$$

A similar answer can be obtained for $a_{2}$

$$
a_{1}=\left[D_{1} e^{i \gamma z}+D_{2} e^{-i \gamma z}\right] e^{\left.-i \bar{\beta}-\frac{K}{2}\right) z} .
$$

Substituting the solutions for $a_{1}$ and $a_{2}$ back into the original equations, solutions can be obtained for the constants $C_{1}, C_{2}, D_{1}$, and $D_{2}$

$$
\begin{array}{r}
D_{1}=\frac{\delta-\gamma}{\kappa_{12}^{+1}} C_{1}, \\
D_{2}=\frac{\delta+\gamma}{\kappa_{12}^{+1}} C_{2}, \\
C_{1}=\frac{\delta-\gamma}{2 \gamma} a_{1}(0)-\frac{\kappa_{12}^{+1}}{2 \gamma} a_{2}(0), \\
C_{2}=-\frac{\delta-\gamma}{2 \gamma} a_{1}(0)+\frac{\kappa_{12}^{+1}}{2 \gamma} a_{2}(0) .
\end{array}
$$

With the coefficients in Equation B.7, the solution can be expressed in matrix form below

$$
T=\left(\begin{array}{cc}
{\left[\cos (\gamma z)+\frac{i \delta}{\gamma} \sin (\gamma z)\right] e^{-\left(\bar{\beta}+\frac{K}{2}\right) z}} & \frac{-i \kappa_{12}^{+1}}{\gamma} \sin (\gamma z) e^{-\bar{\beta}+\frac{K}{2} z} \\
\frac{-i \kappa_{12}^{-1}}{\gamma} \sin (\gamma z) e^{-\bar{\beta}-\frac{K}{2} z} & {\left[\cos (\gamma z)-\frac{i \delta}{\gamma} \sin (\gamma z)\right] e^{-\left(\bar{\beta}-\frac{K}{2}\right) z}}
\end{array}\right)
$$


For a contra directional coupler (i.e. coupling between forward and backwards propagating waves) the initial conditions are as follows below

$$
\begin{aligned}
& a(0)=\left(\begin{array}{l}
1 \\
r
\end{array}\right), \\
& a(L)=\left(\begin{array}{l}
t \\
0
\end{array}\right) .
\end{aligned}
$$

In the above equations, $\mathrm{r}$ and $\mathrm{t}$ are the field reflection and transmission coefficients respectively. The intensity reflection and transmission coefficients are then $|r|^{2}$ and $|t|^{2}$. For a system with two grating reflectors is then represented by

$$
\left(\begin{array}{l}
t \\
0
\end{array}\right)=T\left(\begin{array}{l}
1 \\
r
\end{array}\right)
$$

In this case, the matrix represented by $\mathrm{T}$ is actually a product of three matrices; the left grating, cavity matrix and the right grating. The left and right gratings both obey the T matrix from above. The form of $\mathrm{T}$ is shown in Equation B.8

$$
T=T_{L} T_{M} T_{R}
$$

The matrix for $T_{M}$ acts like a plane wave transfer matrix, seen below

$$
T_{M}=\left(\begin{array}{cc}
e^{-i k_{0} n_{e f f} L} & 0 \\
0 & e^{i k_{0} n_{e f f} L}
\end{array}\right)
$$

From this point, standard tranfer matrix methods (TMM) can be applied to study the spectral response of the system. 


\section{APPENDIX C}

\section{ARBITRARY GEOMETRY CODE}

Below is an example of code used to create arbitrary geometries from .stl files.

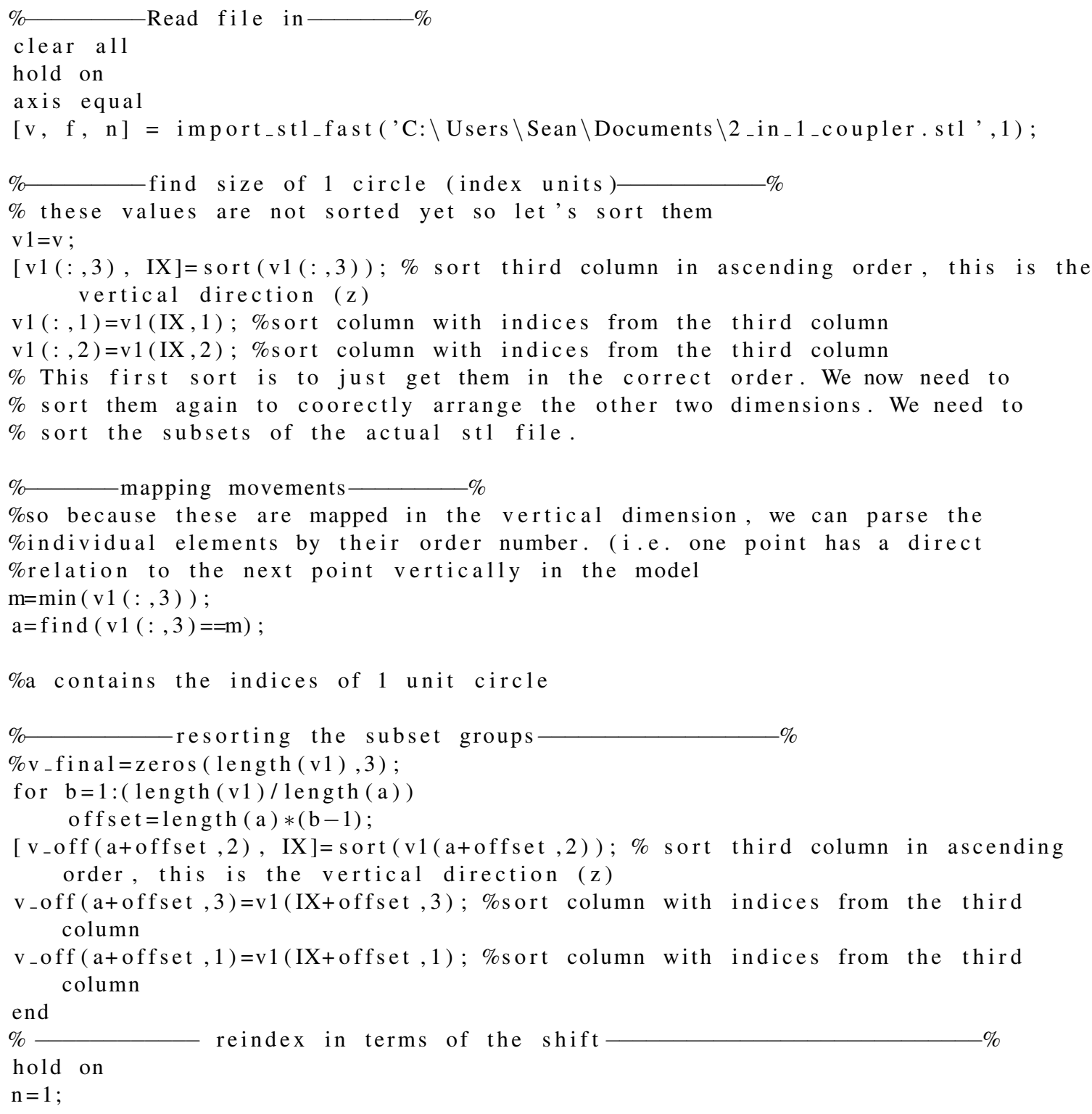




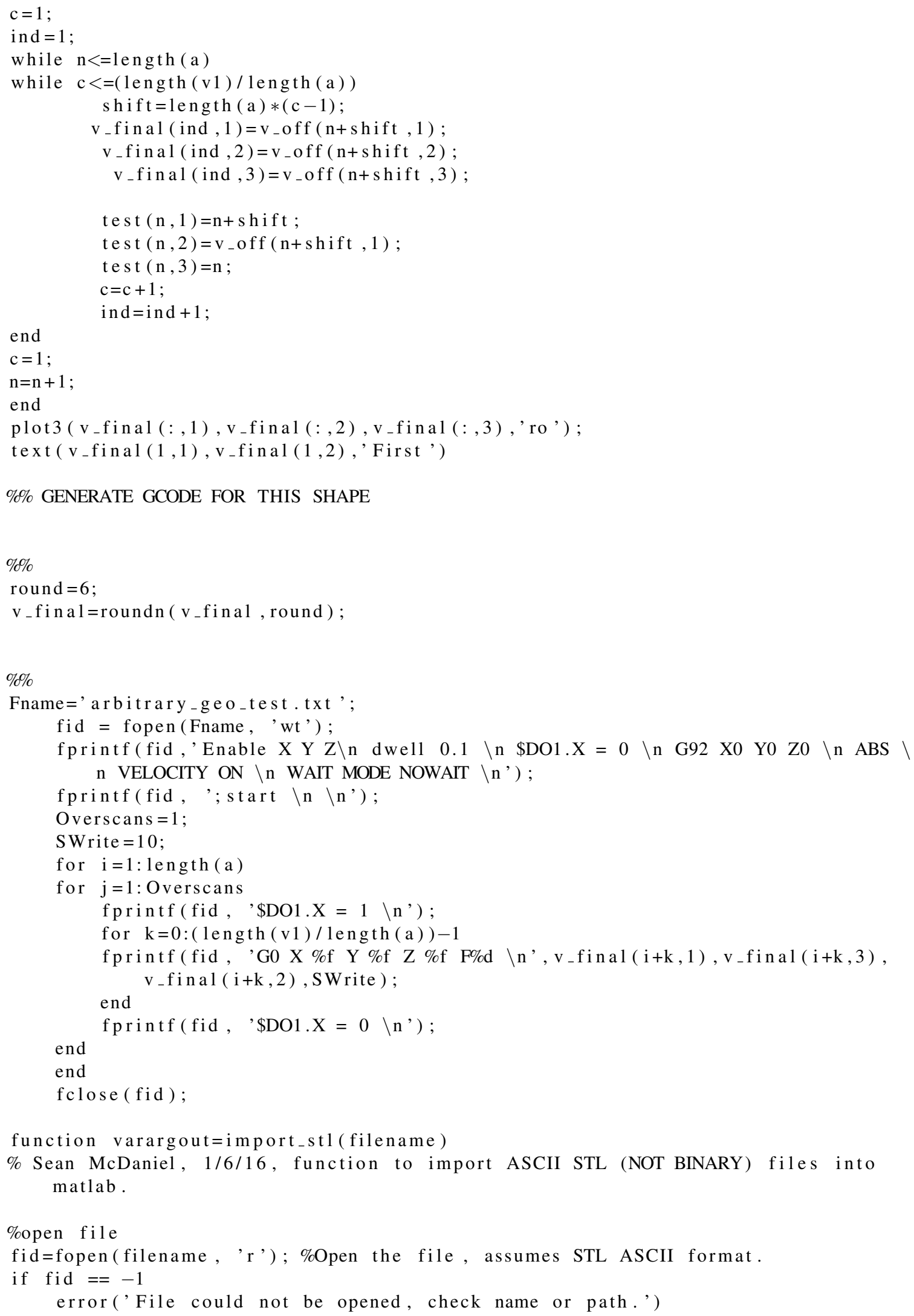


end

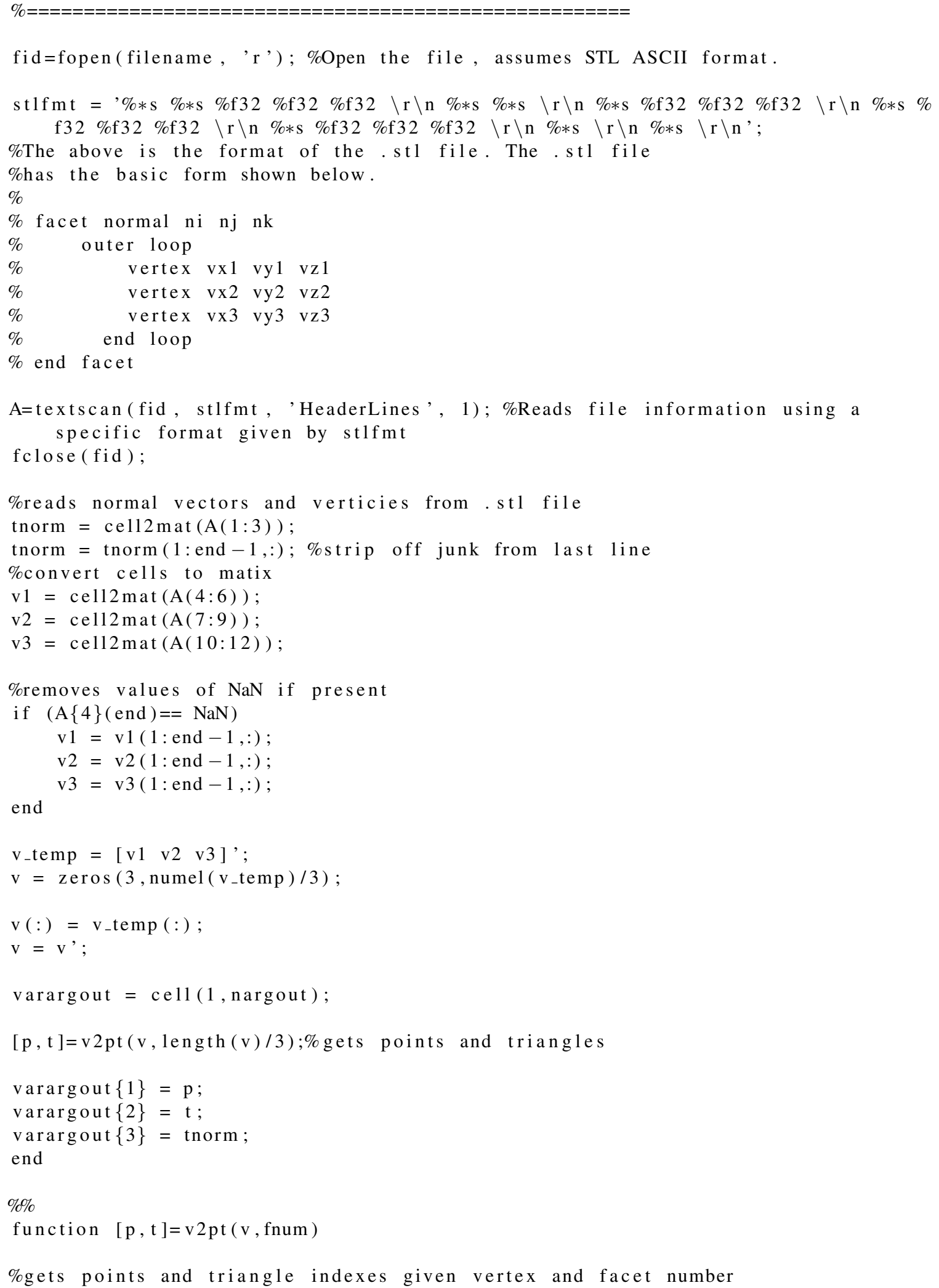


$\mathrm{c}=\operatorname{size}(\mathrm{v}, 1)$;

$\%$ triangles with vertex id data

$\mathrm{t}=\mathrm{zeros}(3$, fnum $)$;

$\mathrm{t}(:)=1: \mathrm{c}$;

\%keep unique vertex values, if not, model won't sort correctly

$[\mathrm{p}, \mathrm{i}, \mathrm{j}]=$ unique $\left(\mathrm{v},{ }^{\prime}\right.$ rows $\left.{ }^{\prime}\right) ; \%$ now $\mathrm{v}=\mathrm{p}(\mathrm{j}) \mathrm{p}(\mathrm{i})=\mathrm{v}$;

$\mathrm{t}(:)=\mathrm{j}(\mathrm{t}(:))$;

$\mathrm{t}=\mathrm{t}$,

end 


\section{APPENDIX D}

\section{RATE EQUATION CODE}

Below is an example of the code used to solve transition metal rate equations.

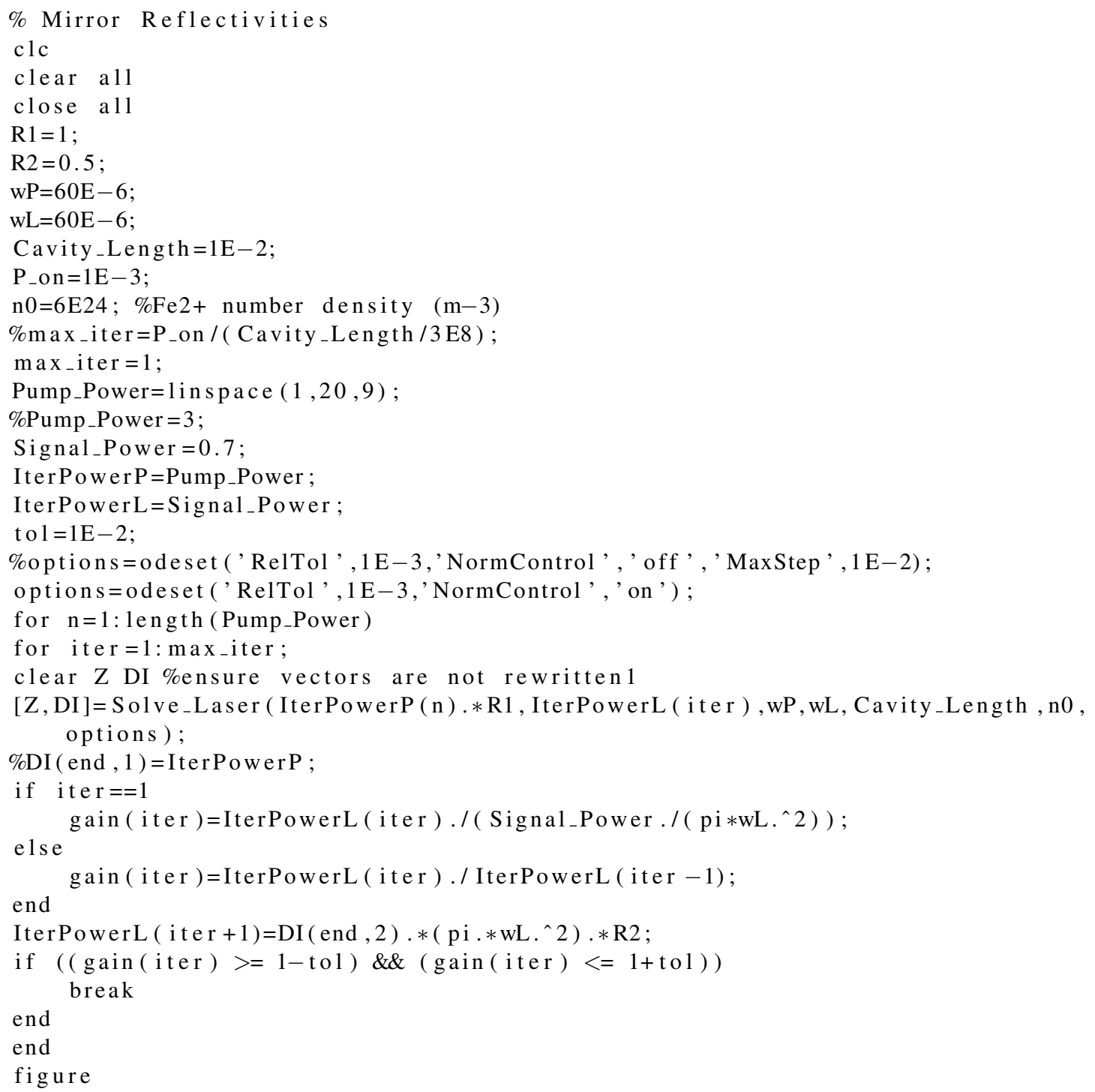




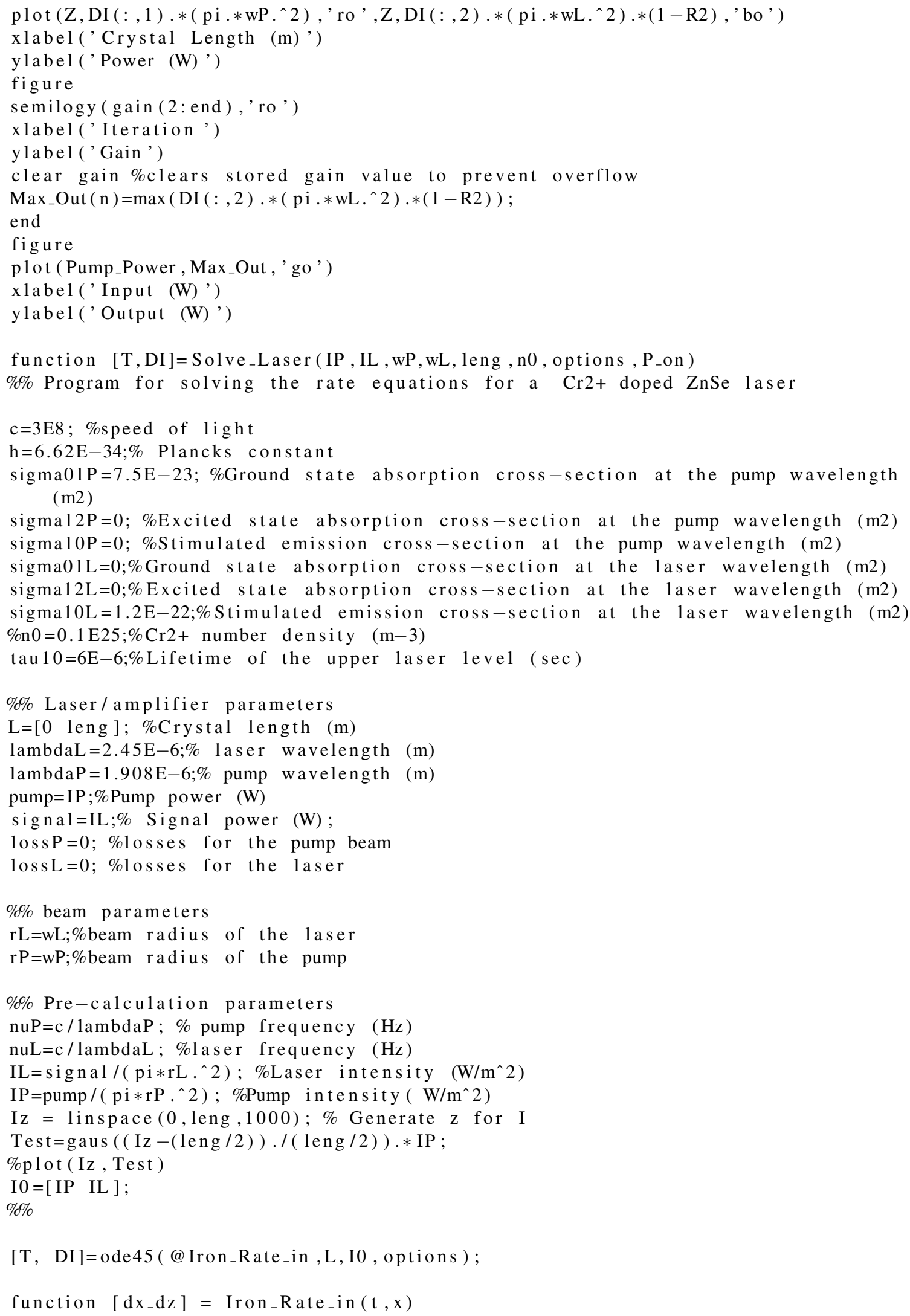


top $=(\mathrm{x}(1) . /(\mathrm{h} . * \mathrm{nuP})) . * \operatorname{sigma} 01 \mathrm{P} . * \mathrm{n} 0 ;$

bottom $=(\mathrm{x}(1) . /(\mathrm{h} . * \mathrm{nuP})) . *(\operatorname{sigma01P}+\operatorname{sigma10P})+(\mathrm{x}(2) . /(\mathrm{h} . * \mathrm{nuL})) . * \operatorname{sigma} 10 \mathrm{~L}+$ $(1 / \operatorname{tau} 10)$;

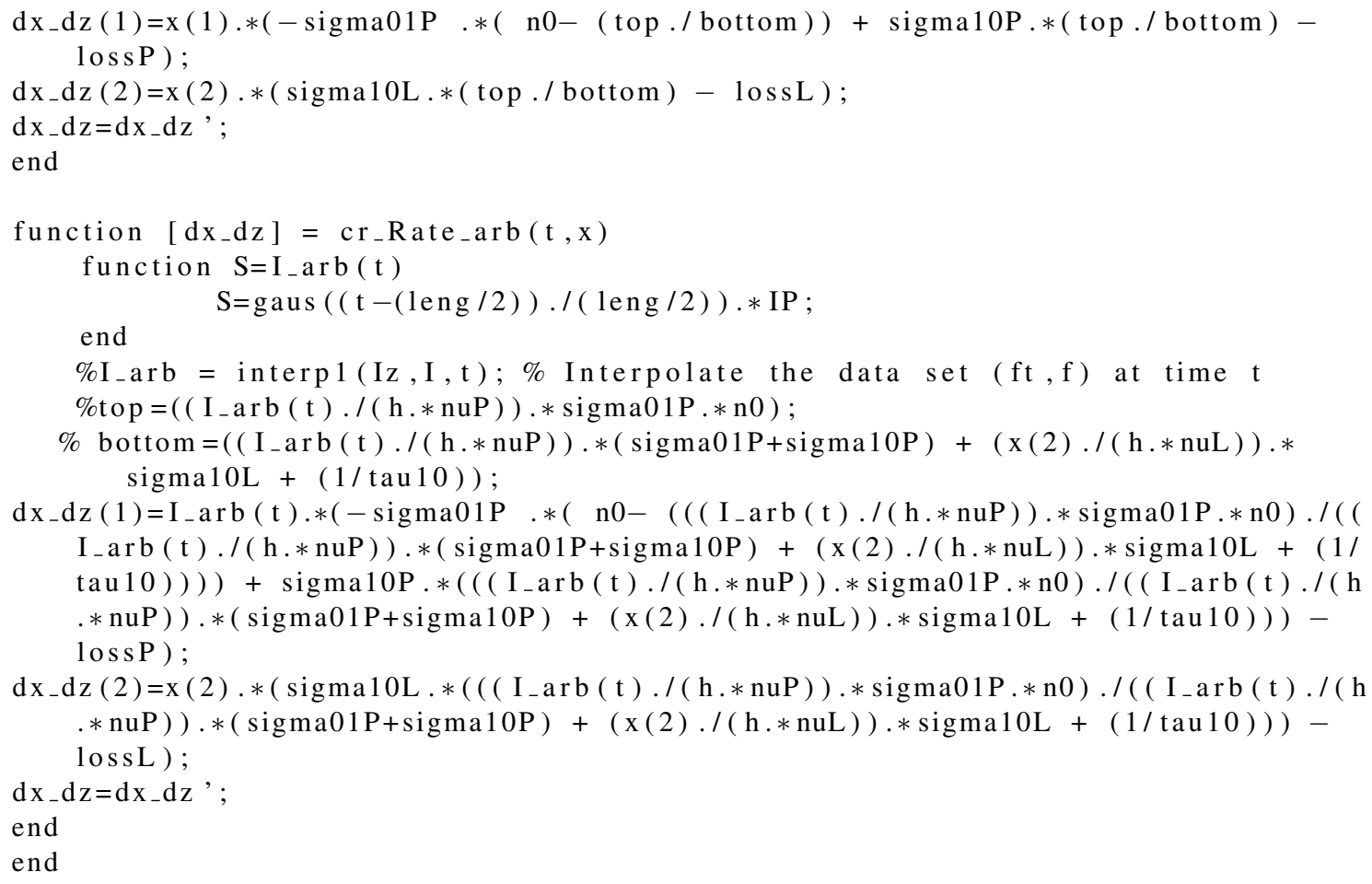

